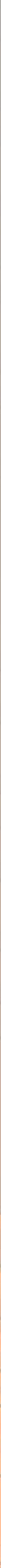




\title{
Double Dirichlet series and quantum unique ergodicity of weight one-half Eisenstein series
}

\author{
Yiannis N. Petridis, Nicole Raulf and Morten S. Risager
}

The problem of quantum unique ergodicity (QUE) of weight $\frac{1}{2}$ Eisenstein series for $\Gamma_{0}(4)$ leads to the study of certain double Dirichlet series involving $\mathrm{GL}_{2}$ automorphic forms and Dirichlet characters. We study the analytic properties of this family of double Dirichlet series (analytic continuation, convexity estimate) and prove that a subconvex estimate implies the QUE result.

\section{Introduction}

An important problem of quantum chaos is to describe the behavior of eigenfunctions of Laplacians $\phi_{\lambda}$ with eigenvalue $\lambda$, as $\lambda \rightarrow \infty$. This problem has a rich and interesting history; see [Shnirelman 1974; Zelditch 1987; Colin de Verdière 1985; Zelditch 1992; Lindenstrauss 2006; Soundararajan 2010a], for example. For the weight 0 Eisenstein series $E(z, s)$ on the surface $\mathrm{SL}_{2}(\mathbb{Z}) \backslash \mathbb{H}$, Luo and Sarnak [1995] determined the asymptotic behavior of the measures

$$
d \mu_{t}(z)=\left|E\left(z, \frac{1}{2}+i t\right)\right|^{2} d \mu(z)
$$

on compact sets. Here $d \mu(z)=d x d y / y^{2}$ denotes the volume element corresponding to the hyperbolic metric on the upper half-plane $\mathbb{H}$. The main input in doing so was subconvex bounds on certain standard $\mathrm{GL}_{1}$ and $\mathrm{GL}_{2} L$-functions, namely the Riemann zeta function and the $L$-function of a Maaß cusp form. Their work was later generalized to the corresponding micro-local lifts [Jakobson 1994] and other arithmetic symmetric spaces [Koyama 2000; Truelsen 2011]. Also for these generalizations, subconvex bounds were at the heart of the proofs. In [Petridis et al. 2013] we studied similar questions for scattering states.

In this paper we study the analogous problem for Eisenstein series of weight $\frac{1}{2}$. To be precise: Let $E\left(z, s, \frac{1}{2}\right)$ be the weight $\frac{1}{2}$ Eisenstein series at the cusp infinity for

Petridis was supported by NSF grant DMS-0401318. Raulf was partly supported by the Labex CEMPI (ANR-11-LABX-0007-01). Risager was supported by a Sapere Aude grant from the Danish Natural Science Research Council.

MSC2010: primary 58J51; secondary 11F68, 11F72.

Keywords: quantum limits, Eisenstein series, multiple Dirichlet series. 
the group $\Gamma=\Gamma_{0}$ (4) (see Section 3). We study the limiting behavior as $|t| \rightarrow \infty$ of

$$
d \mu_{t}(z)=\left|E\left(z, \frac{1}{2}+i t, \frac{1}{2}\right)\right|^{2} d \mu(z) .
$$

Since the Fourier coefficients $\phi_{n}\left(s, \frac{1}{2}\right)$ of $E\left(z, \frac{1}{2}+i t, \frac{1}{2}\right)$ are essentially values of Dirichlet $L$-functions on the critical line - see (3-3) - and, therefore, are not multiplicative, the problem is much harder. The Rankin-Selberg convolutions that appear are not factored into standard $L$-functions. Instead, we find that certain double Dirichlet series play a crucial role. The relevant double Dirichlet series are the following.

Let $\chi, \chi^{\prime}$ be characters mod 8 , and let $t_{n}$ be either the eigenvalue of the Hecke operator $T_{n}$ for a weight $0 \mathrm{Maaß}$ form $\psi$ on $\Gamma_{0}(4) \backslash \mathbb{W}$ or $t_{n}=\tau(n)$ be the divisor function. Let $s_{0}\left(1-s_{0}\right)$ be the corresponding Laplace eigenvalue of $\psi$, with $\Re\left(s_{0}\right) \geq \frac{1}{2}$, and if $t_{n}=\tau(n)$ let $s_{0}=\frac{1}{2}$.

We then define

$$
Z\left(s, w, \chi, \chi^{\prime}\right)=\zeta_{2}(4 s-1) \sum_{\substack{n=1 \\(n, 2)=1}}^{\infty} \frac{\chi(n) t_{n} L^{*}\left(2 w-\frac{1}{2}, n, \chi^{\prime}\right)}{n^{s-w+\frac{1}{2}}},
$$

where $L^{*}(w, n, \chi)=q(w, n, \chi) L_{2}\left(w, \chi_{n_{0}} \chi\right)$. Here $n_{0}$ is the squarefree part of $n$, $\chi_{n_{0}}(c)=\left(\frac{n_{0}}{c}\right)$ and $L_{2}\left(w, \chi_{n_{0}} \chi\right)$ is the standard $L$-function with the 2-factor removed. The functions $q(w, n, \chi)$ are explicitly given so-called "correction polynomials"; see (2-7) below. The function $L^{*}(w, n, \chi)$ may seem strange at first, but it occurs naturally as the $n$-th Fourier coefficient of the Eisenstein series of weight $\frac{1}{2}$, and it has many nice properties. See, for example, [Shimura 1973] or Section 3 below.

Friedberg and Hoffstein [1995] have studied a Rankin-Selberg integral (see (3-13) below) which turns out to be a linear combination of $Z\left(s, w, \chi, \chi^{\prime}\right)$ and $Z\left(s, w, \chi, \chi_{4} \chi^{\prime}\right)$, where $\chi_{4}$ is the primitive character mod 4 . They observed that this admits meromorphic continuation and that certain linear combinations have a pole at $(s, w)=\left(\frac{3}{4}, \frac{3}{4}\right)$ (in our normalization). They did this in order to prove nonvanishing of quadratic twists of $\mathrm{GL}_{2}-L$-functions at the central point.

Furthermore similar series with higher-order twists instead of the quadratic characters $\chi_{n_{0}}$ were studied by Brubaker, Bucur, Chinta, Frechette and Hoffstein [Brubaker et al. 2004] in order to prove nonvanishing of higher-order twists. To understand the new series $Z\left(s, w, \chi, \chi^{\prime}\right)$ we follow essentially the program introduced in [Bump et al. 1996] to prove the following.

The series defining $Z\left(s, w, \chi, \chi^{\prime}\right)$ converges absolutely and uniformly in certain regions in $\mathbb{C}^{2}$, and hence defines an analytic function there. The functions $Z\left(s, w, \chi, \chi^{\prime}\right)$ admit meromorphic continuation to $\mathbb{C}^{2}$ and they satisfy a group of functional equations generated by

$$
\alpha:(s, w) \mapsto(s, 1-w), \quad \beta:(s, w) \mapsto(w, s) .
$$


The functions $Z\left(s, w, \chi, \chi^{\prime}\right)$ grow at most polynomially for $(\Re(s), \Re(w))$ in compact sets. For the precise form of the functional equations we refer to Theorems 2.11 and 2.13. The group of functional equations is isomorphic to the dihedral group of order 8. A similar result for higher-order twists may be found in [Brubaker et al. 2004].

We want to investigate the growth of $Z\left(s, w, \chi, \chi^{\prime}\right)$ in $s$ and $w$. The notions of analytic conductor and subconvexity are not completely well established for general multiple Dirichlet series. Certain cases are dealt with in [Blomer 2011; Blomer et al. 2014] but a general theory is missing.

To define these notions in the present case we note that when $\Re(s), \Re(w)>\frac{3}{4}$ the function $Z\left(s, w, \chi, \chi^{\prime}\right)$ has a representation

$$
Z\left(s, w, \chi, \chi^{\prime}\right)=\sum_{\substack{c=1 \\(c, 2)=1}}^{\infty} \frac{\chi^{\prime}(c) L^{* *}\left(s-w+\frac{1}{2}, \psi, c, \chi\right)}{c^{2 w-1 / 2}},
$$

where $L^{* *}(s, \psi, c, \chi)=Q^{*}(s, c, \chi) L_{2}\left(s, \psi \otimes \tilde{\chi}_{c_{0}} \chi\right)$ (see (2-19) and Theorem 2.13). Here $c_{0}$ is the squarefree part of $c, \tilde{\chi}_{c_{0}}(n)=\left(\frac{n}{c_{0}}\right)$ and $L_{2}\left(s, \psi \otimes \tilde{\chi}_{c_{0}} \chi\right)$ is the standard $L$-function with the 2 -factor removed. The functions $Q^{*}(s, c, \chi)$ are explicitly given so-called "correction polynomials"; see (2-20) below.

When proving bounds on standard $L$-functions one usually normalizes the coefficients to be essentially bounded, at least on average. In our case it is not so clear how to do that since the true size of $L^{* *}(s, \psi, c, \chi)$ is known only conjecturally. If the generalized Lindelöf hypothesis is true the coefficients of the series (1-3) are essentially bounded. We investigate what happens when this is true on average (over $c$ ). To be precise: we want to know what bound on $Z\left(s, w, \chi, \chi^{\prime}\right)$ can be proved if we assume that the coefficients are essentially bounded, i.e., if

$$
\sum_{\substack{c \leq X \\(c, 2)=1}}\left|L^{* *}(s, \psi, c, \chi)\right|=O\left(X^{1+\epsilon}(1+|s|)^{\epsilon}\right) .
$$

Using the properties of $Q^{*}(s, c, \chi)$ we will see that this follows from assuming

$$
\sum_{\substack{1 \leq c_{0} \leq X \\ c_{0} \text { odd, } \\ \text { squarefree }}}\left|L_{2}\left(s, \psi \otimes \tilde{\chi}_{c_{0}} \chi\right)\right|^{2}=O\left(X^{1+\epsilon}(1+|s|)^{\epsilon}\right) \quad \text { when } \Re(s)=\frac{1}{2} .
$$

Also, it is easy to see that (1-4) implies (1-5) with the exponent 2 replaced by a 1 . In particular it implies the generalized Lindelöf hypothesis in the $t$ parameter.

We now define the analytic conductor of $Z\left(\frac{1}{2}+i t, \frac{1}{2}+i u, \chi, \chi^{\prime}\right)$ to be

$$
\mathfrak{q}(t, u)=(1+|t|)(1+|t+u|)^{2}(1+|u|) .
$$


Using an approximate functional equation argument for $Z\left(s, w, \chi, \chi^{\prime}\right)$ we can prove the following bound on the critical line.

Theorem 1.1. Assume (1-4). Then

$$
Z\left(\frac{1}{2}+i t, \frac{1}{2}+i u, \chi, \chi^{\prime}\right)=O_{\psi}\left(\mathfrak{q}(t, u)^{\frac{1}{4}+\varepsilon}\right) .
$$

Unconditionally,

$$
Z\left(\frac{1}{2}+i t, \frac{1}{2}+i u, \chi, \chi^{\prime}\right)=O_{\psi}\left(\left(\mathfrak{q}(t, u)(1+|t-u|)^{2}\right)^{\frac{1}{4}+\varepsilon}\right) .
$$

Remark 1.2. We call the unconditional bound (1-8) the trivial bound. The conditional bound (1-7) is called the convexity bound. Any bound $O\left(\mathfrak{q}(t, u)^{\frac{1}{4}-\delta}\right)$ with $\delta>0$ is called a subconvex bound with saving $\delta$. If $\delta=\frac{1}{4}-\varepsilon$ is permitted, we say that $Z\left(s, w, \chi, \chi^{\prime}\right)$ admits a Lindelöf-type bound. In the theory of $L$-functions, the notion of convexity and subconvexity is standard and has numerous applications; see, e.g., [Iwaniec and Kowalski 2004].

Remark 1.3. We note that even proving the trivial bound requires strong input. In particular, in order to prove Theorem $1.1(1-8)$, we need the Lindelöf hypothesis on average in the conductor aspect for $L\left(s, \chi_{n}\right)$, and the convexity estimate in the $s$ aspect. This bound is available, as follows from Heath-Brown's famous large sieve inequality for quadratic characters (2-28); see (2-29) below.

Also, we note that we can prove unconditionally (see Lemma 3.2 below) that, if $\left\{t_{n}\right\}$ comes from a cusp form,

$$
Z\left(\frac{1}{2}+i t, \frac{1}{2}-i t, \chi, \chi^{\prime}\right)+b Z\left(\frac{1}{2}+i t, \frac{1}{2}-i t, \chi, \chi^{\prime}\right)=O_{\psi}\left(\mathfrak{q}(t,-t)^{\frac{1}{4}+\varepsilon}\right) .
$$

Here $b$ is the product of the sign of $\chi$ and the sign of the cusp form. We note that this is of the same order as the convexity estimate above without assuming (1-4).

Remark 1.4. For special configurations of $s, w$ (in our case $s-w$ constant) the trivial bound and the convexity bound coincide. This is because, in this case, (1-5) follows from Heath-Brown's estimate (2-30).

We emphasize that our notion of convexity is different from that of Blomer, Goldmakher and Louvel [Blomer 2011; Blomer et al. 2014]. What we call the trivial bound corresponds to what they call the convexity bound.

Remark 1.5. Even though we cannot prove it, it is not unreasonable to expect subconvexity for $Z\left(s, w, \chi, \chi^{\prime}\right)$ ! Double Dirichlet series similar to $Z\left(s, w, \chi, \chi^{\prime}\right)$ with degree-one $L$-functions as coefficients - are known to satisfy subconvex bounds [Blomer 2011; Blomer et al. 2014]. (Blomer et al. [2014] consider a configuration such that the bound they prove would be considered a subconvex bound also by our definition. Likewise the bound proved in [Blomer 2011, Theorem 1] is a subconvex bound by our definition if one restricts to $s=\frac{1}{2}$ or $w=\frac{1}{2}$.) 
Furthermore, it is known that on average the double Dirichlet series considered by Blomer admits Lindelöf-type bounds [Blomer 2011, Theorem 2] in the $(s, w)$ aspect. In the conductor aspect (which is here the conductor related to the form with eigenvalues $\left\{t_{n}\right\}$ ), Hoffstein and Kontorovich [2010, (1.23)] conjecture Lindelöf-type bounds to hold.

Theorem 1.6. Assume that, for all $\chi, \chi^{\prime},\left\{t_{n}\right\}$ the function $Z\left(s, w, \chi, \chi^{\prime}\right)$ admits a subconvex bound. Then, for any compact Jordan measurable subsets $A$ and $B$ of $\Gamma \backslash \mathbb{V}$, we have

$$
\frac{\int_{A}\left|E\left(z, \frac{1}{2}+i t, \frac{1}{2}\right)\right|^{2} d \mu(z)}{\int_{B}\left|E\left(z, \frac{1}{2}+i t, \frac{1}{2}\right)\right|^{2} d \mu(z)} \rightarrow \frac{\operatorname{vol}(A)}{\operatorname{vol}(B)} \quad \text { as }|t| \rightarrow \infty .
$$

Remark 1.7. Theorem 1.6 is the analogue of the Luo-Sarnak theorem [1995] for the weight 0 Eisenstein series. Their theorem, however, is unconditional, as in their case subconvex bounds for standard $\mathrm{GL}_{1}$ and $\mathrm{GL}_{2}-L$-functions are readily available. As in that paper, we really prove - conditionally on any subconvex bound - the asymptotic result

$$
\int_{A}\left|E\left(z, \frac{1}{2}+i t, \frac{1}{2}\right)\right|^{2} d \mu(z) \sim \frac{4}{\operatorname{vol}(\Gamma \backslash \mathbb{M})} \operatorname{vol}(A) \log |t| \quad \text { as }|t| \rightarrow \infty .
$$

In contrast to the case of quantum unique ergodicity of Maaß cusp forms, the rate of convergence in (1-10) is very slow. As in [Luo and Sarnak 1995] one can prove $O(\log t / \log \log t)$.

It is understood in many arithmetic cases that the equidistribution of masses is implied by subconvexity bounds for appropriate $L$-functions of degree 8 ; see, e.g., [Sarnak 2011; Soundararajan 2010b; Nelson et al. 2014].

Remark 1.8. The structure of the paper is as follows. In Section 2 we study the double Dirichlet series $Z\left(s, w, \chi, \chi^{\prime}\right)$ which arise when we address QUE of the weight $\frac{1}{2}$ Eisenstein series $E\left(z, s, \frac{1}{2}\right)$. In Section 3 we review the theory for $E\left(z, s, \frac{1}{2}\right)$ with explicit computations. In Section 4 , which is the main section of the paper, we analyze (1-10) by splitting it into a cuspidal contribution and incomplete Eisenstein series contributions. For example, in the cuspidal space we find that, for a cusp form $\psi$ with eigenvalue $s_{0}\left(1-s_{0}\right)$, the integral

$$
\int_{\Gamma \backslash \mathbb{H}} \psi(z)\left|E\left(z, \frac{1}{2}+i t, \frac{1}{2}\right)\right|^{2} d \mu(z)
$$

equals a linear combination of terms of the form

$$
c_{\chi, \chi_{ \pm}^{\prime}}(s, w) Z\left(s, w, \chi, \chi^{\prime}\right) \frac{1}{\Gamma\left(w \pm \frac{1}{4}\right)} \int_{0}^{\infty} W_{0, s_{0}-\frac{1}{2}}(2 y) W_{ \pm \frac{1}{4}, w-\frac{1}{2}}(2 y) y^{s-1} \frac{d y}{y}
$$


evaluated at $(s, w)=\left(\frac{1}{2}+i t, \frac{1}{2}-i t\right)$. Here $c_{\chi, \chi_{ \pm}^{\prime}}(s, w)$ are functions which can easily be understood when $\Re(w)=\Re(s)=\frac{1}{2}$, and $W_{\mu, \nu}$ are Whittaker functions. In the Appendix we analyze the Mellin transform of the product of Whittaker functions.

We can then deal with (1-12) using bounds on $Z\left(s, w, \chi, \chi^{\prime}\right)$. To deal with the cuspidal space we need subconvexity for $Z\left(\frac{1}{2}+i t, \frac{1}{2}-i t, \chi, \chi^{\prime}\right)$, with $t_{n}$ corresponding to Hecke eigenvalues for Maaß forms. For the incomplete Eisenstein series a similar analysis shows that we need the same type of bound for $t_{n}=\tau(n)$, the divisor function, for all configurations of $s$ and $w$. We also use Zagier's theory of Rankin-Selberg integrals for functions not of rapid decay.

Remark 1.9. Although the analytic continuation of

$$
I(s, w)=\int_{\Gamma \backslash \mathbb{\sharp}} \psi(z) E\left(z, w, \frac{1}{2}\right) \overline{E\left(z, \bar{s}, \frac{1}{2}\right)} d \mu(z)
$$

(of which (1-11) is a special case) follows from the well-known analytic properties of $E\left(z, w, \frac{1}{2}\right)$, its growth/decay properties jointly in $(s, w)$ are less clear. This is why we have to unfold and eventually analyze $Z\left(s, w, \chi, \chi^{\prime}\right)$ to see that the above integral is $O\left(|t|^{-\delta}\right)$ for $s=1-w=\frac{1}{2}+i t$ when $|t| \rightarrow \infty$, assuming subconvexity with saving $\delta$. The Maaß-Selberg relation gives an upper bound (see, e.g., (3-15) below), but this is not good enough to prove Theorem 1.6.

Remark 1.10. One could speculate whether the implication in Theorem 1.6 could be reversed, i.e., to what extent bounds on integrals like (1-11) would imply bounds on $Z\left(s, w, \chi, \chi^{\prime}\right)$ via the expression (1-12). Such speculation is problematic at least for the following reason. We have good control over the asymptotics of the Mellin transform (see, e.g., Lemma A.1) but since integrals like (1-11) are linear combinations of terms of the form (1-12), we cannot conclude from bounds on integrals like (1-11) the same bounds on the individual summands. We elaborate on this in Lemma 3.2 and Remark 3.3 below.

\section{A double Dirichlet series}

In this section we define and prove various properties of the double Dirichlet series. To derive its meromorphic continuation and functional equation we proceed as in [Brubaker et al. 2004], but with some simplifications and refinements. We show, for instance, that knowing optimal bounds towards the Ramanujan-Petersson conjecture is not necessary to get optimal regions of convergence. To prove the convexity bounds we use a combination of techniques from [Blomer 2011; Blomer et al. 2014]. Although the techniques we use are certainly known to the experts in the field, we were not able to find precise enough statements in the existing literature for the double Dirichlet series (1-2). 
We start by introducing some notation and deriving some basic results about Gauss sums and Dirichlet series involving Gauss sums.

Let $\left\{t_{n}\right\}_{n \in \mathbb{N}}$ be the coefficients of the normalized $L$-function of a self-dual $\mathrm{GL}_{2}$ automorphic form $\psi$. For good primes - and we assume that only $p=2$ could potentially be a bad prime - the Satake parameters $\alpha_{p}, \beta_{p}$ satisfy $\alpha_{p}+\beta_{p}=t_{p}$, $\alpha_{p} \cdot \beta_{p}=1$ and

$$
t_{p^{\lambda}}=\sum_{j=0}^{\lambda} \alpha_{p}^{j} \beta_{p}^{\lambda-j}=\frac{\alpha_{p}^{\lambda+1}-\beta_{p}^{\lambda+1}}{\alpha_{p}-\beta_{p}} .
$$

The Fourier coefficients satisfy the Ramanujan-Petersson conjecture on average, since the Rankin-Selberg method gives

$$
\sum_{|n| \leq X}\left|t_{n}\right|^{2} \sim C X
$$

as $X \rightarrow \infty$. Here $C$ is an explicit constant; see, e.g., [Iwaniec 2002, (8.15)]. The corresponding $p$-factor, i.e., the local $L$-function, is given by

$$
L^{(p)}(s, \psi)=\sum_{\lambda=0}^{\infty} \frac{t_{p^{\lambda}}}{p^{\lambda s}}=\left(1-t_{p} p^{-s}+p^{-2 s}\right)^{-1}=\left(1-\alpha_{p} p^{-s}\right)^{-1}\left(1-\beta_{p} p^{-s}\right)^{-1} .
$$

Similar but easier identities and estimates are true for the divisor function $t_{n}=\tau(n)$, where $\alpha_{p}=\beta_{p}=1$.

For any $L$-function we will write $L^{(p)}(s)$ for its corresponding $p$-factor and $L_{2}(s)$ for the $L$-function with the 2 -factor removed.

2A. Gauss sums and some related series. We now recall a few basic relevant results about Gauss sums for real characters. Let $n, d$ be integers with $d$ odd and positive and let $\left(\frac{n}{d}\right)$ be the Jacobi-Legendre symbol

$$
\left(\frac{n}{d}\right)=\prod_{p^{v} \| d}\left(\frac{n}{p}\right)^{v}
$$

where for an odd prime $p$ we denote by $\left(\frac{n}{p}\right)$ the usual Legendre symbol. The symbol $\left(\frac{n}{d}\right)$ is then extended to all odd $d \in \mathbb{Z}$ as in [Shimura 1973, p. 442]; see also [Koblitz 1984, p. 147, 187-188].

For an integer $n$ and a positive odd integer $d$ we define Gauss sums

$$
G_{n}(d):=\sum_{m \bmod d}\left(\frac{m}{d}\right) e\left(\frac{n m}{d}\right) .
$$

Here $e(x)=e^{2 \pi i x}$. Gauss ingeniously proved that for odd squarefree $d$ we have $G_{1}(d)=\varepsilon_{d} \sqrt{d}$, where $\varepsilon_{d}=1$ if $d \equiv 1(\bmod 4)$ and $\varepsilon_{d}=i$ if $d \equiv-1(\bmod 4)$. 
Quadratic reciprocity states that for relatively prime odd positive integers $n, d$,

$$
\left(\frac{n}{d}\right)\left(\frac{d}{n}\right)=(-1)^{\frac{n-1}{2} \frac{d-1}{2}} .
$$

It is elementary to verify that the right-hand side equals $\varepsilon_{n} \varepsilon_{d} / \varepsilon_{n d}$. For odd $d$ it turns out to be convenient to consider

$$
H_{n}(d):=\varepsilon_{d}^{-1} G_{n}(d)
$$

Proposition 2.1. The function $H_{n}(d)$ has the following properties:

(1) For fixed $n, H_{n}(d)$ is multiplicative, i.e., if $d_{1}, d_{2}$ are coprime odd positive integers, then

$$
H_{n}\left(d_{1} d_{2}\right)=H_{n}\left(d_{1}\right) H_{n}\left(d_{2}\right)
$$

(2) If $\left(n_{1}, d\right)=1$, then

$$
H_{n_{1} n_{2}}(d)=\left(\frac{n_{1}}{d}\right) H_{n_{2}}(d) .
$$

(3) Let $\alpha, \beta$ be nonnegative integers and let $p$ be an odd prime. Then

$$
H_{p^{\alpha}}\left(p^{\beta}\right)= \begin{cases}\phi\left(p^{\beta}\right) & \text { if } \alpha \geq \beta, \beta \equiv 0(\bmod 2), \\ p^{\beta-\frac{1}{2}}\left(\delta_{\beta \equiv 1(\bmod 2)}-p^{-\frac{1}{2}} \delta_{\beta \equiv 0(\bmod 2)}\right) & \text { if } \alpha=\beta-1, \\ 0 & \text { otherwise. }\end{cases}
$$

Proof. (1) follows from the Chinese remainder theorem and quadratic reciprocity;

(2) from the fact that if $\left(n_{1}, d\right)=1$ then $n_{1} m$ runs through a set of representatives $\bmod d$; and (3) from elementary considerations.

We now compute

$$
\sum_{\substack{c=1 \\(c, 2)=1}}^{\infty} \frac{\chi(c) H_{n}(c)}{c^{2 s}} \text { and } \sum_{\substack{n=1 \\(n, 2)=1}}^{\infty} \frac{t_{n} \chi(n) H_{n}(c)}{n^{s}}
$$

where $\chi$ is a character $\bmod q$ with $q \mid 8$. As we shall see later these sums occur naturally in the Fourier coefficients of the weight $\frac{1}{2}$ Eisenstein series of $\Gamma_{0}(4)$, and in Rankin-Selberg-type integrals formed from these Eisenstein series.

For $n$ odd and positive we denote

$$
\tilde{\chi}_{n}(c)=\left(\frac{c}{n}\right),
$$

which is a character $\bmod n$. When $n$ is squarefree its conductor is $n$.

For $c$ odd we denote

$$
\chi_{n}(c)=\left(\frac{n}{c}\right)
$$


which for $n$ odd and squarefree has an extension to all $c$ which is a character of conductor $|n|$ if $n \equiv 1(\bmod 4)$ and $4|n|$ if $n \equiv 3(\bmod 4)$. See [Koblitz 1984, p. 147, 187-188].

By quadratic reciprocity (2-4) we have, for odd positive $m, n$,

$$
\chi_{n}(m)=\tilde{\chi}_{n}(m) \begin{cases}1 & \text { if } n \equiv 1(\bmod 4), \\ \chi_{4}(m) & \text { if } n \equiv 3(\bmod 4),\end{cases}
$$

where $\chi_{4}$ is the primitive character mod 4 . We can write any nonzero integer $n$ uniquely as $n=n_{0} n_{1}^{2}$, where $n_{0}$ is squarefree and $n_{1}>0$. We define correction polynomials as

$$
q(s, n, \chi)=\prod_{2 \neq p \mid n_{1}} \sum_{\beta=0}^{v_{p}\left(n_{1}\right)} \frac{1-\delta_{\beta<v_{p}\left(n_{1}\right)} \chi_{n_{0}}(p) \chi(p) p^{-s}}{p^{2 \beta\left(s-\frac{1}{2}\right)}},
$$

where $v_{p}$ is the $p$-adic valuation. For $\chi=1$, we sometimes write $q(s, n)=q(s, n, \chi)$.

We define

$$
L^{*}(s, n, \chi)=q(s, n, \chi) L_{2}\left(s, \chi_{n_{0}} \chi\right)
$$

Lemma 2.2. We have

$$
\sum_{\substack{c=1 \\(c, 2)=1}}^{\infty} \frac{\chi(c) H_{n}(c)}{c^{2 s}}=\frac{L^{*}\left(2 s-\frac{1}{2}, n, \chi\right)}{\zeta_{2}(4 s-1)} .
$$

Proof. Using multiplicativity of $H_{n}(d)$ (Proposition 2.1) we see that the sum factors into local factors. For a prime $p \neq 2$ we compute the corresponding factor

$$
R_{p}(s)=\sum_{\beta=0}^{\infty} \frac{\chi\left(p^{\beta}\right) H_{n}\left(p^{\beta}\right)}{p^{\beta 2 s}} .
$$

Write $n=n^{\prime} p^{\alpha}$, where $\left(n^{\prime}, p\right)=1$. Then using Proposition 2.1 (2), (3) we have

$$
R_{p}(s)=\sum_{\beta=0}^{\infty} \frac{\left(\frac{n^{\prime}}{p^{\beta}}\right) \chi\left(p^{\beta}\right) H_{p^{\alpha}}\left(p^{\beta}\right)}{p^{\beta 2 s}}=\sum_{\substack{\beta=0 \\ \beta \text { even }}} \frac{\phi\left(p^{\beta}\right)}{p^{\beta 2 s}}+\frac{\left(\frac{n^{\prime}}{p^{\alpha+1}}\right) \chi\left(p^{\alpha+1}\right) H_{p^{\alpha}}\left(p^{\alpha+1}\right)}{p^{(\alpha+1) 2 s}}
$$

Consider first $\alpha$ even, in which case $\alpha=2 v_{p}\left(n_{1}\right)$. Then we find

$$
R_{p}(s)=1+\sum_{\substack{\beta=1 \\ \beta \text { even }}}^{\alpha} \frac{p^{\beta-1}(p-1)}{p^{\beta 2 s}}+\frac{\chi_{n_{0}}(p) \chi(p) p^{\alpha+\frac{1}{2}}}{p^{(\alpha+1) 2 s}}
$$


noting that $\chi_{n_{0}}(p)=\left(\frac{n^{\prime}}{p}\right)$. By induction we find

$$
R_{p}(s)=\frac{L^{(p)}\left(2 s-\frac{1}{2}, \chi_{n_{0}} \chi\right)}{\zeta^{(p)}(4 s-1)}\left(\sum_{\substack{\beta=0 \\ \beta \text { even }}}^{\alpha} p^{\beta(1-2 s)}-\sum_{\substack{\beta=0 \\ \beta \text { even }}}^{\alpha-2} \chi_{n_{0}}(p) \chi(p) p^{-\left(2 s-\frac{1}{2}\right)} p^{\beta(1-2 s)}\right) .
$$

Here we have used $\chi_{n_{0}}^{2}(p)=1$.

Returning to (2-9), we assume instead that $\alpha$ is odd, in which case $\alpha-1=2 v_{p}\left(n_{1}\right)$. We find that in this case

$$
\begin{aligned}
R_{p}(s) & =1+\sum_{\substack{\beta=1 \\
\beta \text { even }}}^{\alpha} \frac{p^{\beta-1}(p-1)}{p^{\beta 2 s}}+\frac{-p^{\alpha}}{p^{(\alpha+1) 2 s}} \\
& =\left(1-p^{-(4 s-1)}\right) \sum_{\substack{\beta=0 \\
\beta \text { even }}}^{\alpha-1} p^{\beta(1-2 s)},
\end{aligned}
$$

where again we have used induction. Using that, for $\alpha$ odd, $\chi_{n_{0}}(p)=0$, we may write this as

$$
R_{p}(s)=\frac{L^{(p)}\left(2 s-\frac{1}{2}, \chi_{n_{0}} \chi\right)}{\zeta^{(p)}(4 s-1)} \sum_{\substack{\beta=0 \\ \beta \text { even }}}^{\alpha-1} p^{\beta(1-2 s)} .
$$

Since $\chi_{n_{0}}(p)=0$, we arrive at the desired result.

Proposition 2.3. The function $q(s, n, \chi)$ has the following properties:

(1) If $n$ is squarefree, then $q(s, n, \chi)=1$.

(2) If $n=n_{0} n_{1}^{2}$ with $n_{0}$ squarefree and $n_{0}, n_{1}$ odd, then

$$
q(s, n, \chi)=\left(n_{1}^{2}\right)^{\frac{1}{2}-s} q(1-s, n, \chi) .
$$

(3) If $\Re(s) \geq \frac{1}{2}$, then $q(s, n, \chi)=O\left(n^{\epsilon}\right)$ uniformly in $\Re(s)$.

Proof. These statements are all straightforward to verify from the definition. (1) is clear and (2) is easily verified by considering factors. Trivial estimates for $\Re(s) \geq \frac{1}{2}$ lead to $|q(s, n, \chi)| \leq 2^{\#\{p \mid n\}} \tau(n)$, which gives (3).

Write $c=c_{0} c_{1}^{2}$ with $c_{0}$ squarefree and set $v=v_{p}\left(c_{1}\right)$. We then define, for odd $c$,

$$
Q_{\psi}(s, c, \chi)=\prod_{p \mid c_{1}} \frac{t_{p^{2 v}}-t_{p^{2 v-1}} \tilde{\chi}_{c_{0}}(p) \chi(p)\left(p^{1-s}+p^{s}\right) / p+t_{p^{2 v-2}} \tilde{\chi}_{c_{0}}(p)^{2} / p}{p^{2 v\left(s-\frac{1}{2}\right)}} .
$$

Since $\psi$ is fixed, we shall often omit it from the notation and simply write $Q(s, c, \chi)$. We define

$$
L^{*}(s, c, \psi, \chi):=Q_{\psi}(s, c, \chi) L_{2}\left(s, \psi \otimes \tilde{\chi}_{c_{0}} \chi\right) .
$$


Lemma 2.4. Let $c$ be an odd natural number. Then

$$
\sum_{\substack{n=1 \\(n, 2)=1}}^{\infty} \frac{t_{n} \chi(n) H_{n}(c)}{n^{s}}=\sqrt{c} L^{*}(s, c, \psi, \chi)
$$

Proof. A similar computation can be found in [Brubaker et al. 2004, Section 3]. We first show that the Dirichlet series factors into local factors. For $p$ an odd prime, write $c=c^{\prime} p^{l}$ with $\left(c^{\prime}, p\right)=1$, and $m=p^{v_{p}(m)} m / p^{v_{p}(m)}$. Then using Proposition 2.1 (1) and (2) we find

$$
H_{m}(c)=\left(\frac{m / p^{v_{p}(m)}}{p^{l}}\right)\left(\frac{p^{v_{p}(m)}}{c^{\prime}}\right) H_{p^{v_{p}(m)}}\left(p^{l}\right) H_{m / p^{v_{p}(m)}}\left(c^{\prime}\right) .
$$

Writing $m=n p^{\lambda}$, we can write the Dirichlet series as

$$
\begin{aligned}
\sum_{\substack{n=1 \\
(n, 2 p)=1}}^{\infty} \sum_{\lambda=0}^{\infty} \frac{t_{n p^{\lambda}} \chi\left(n p^{\lambda}\right)}{\left(n p^{\lambda}\right)^{s}} & H_{n p^{\lambda}}\left(c^{\prime} p^{l}\right) \\
& =\sum_{\substack{n=1 \\
(n, 2 p)=1}}^{\infty} \frac{t_{n} \chi(n) H_{n}\left(c^{\prime}\right)\left(\frac{n}{p^{l}}\right)}{n^{s}}\left(\sum_{\lambda=0}^{\infty} \frac{t_{p^{\lambda}} \chi\left(p^{\lambda}\right)}{p^{\lambda s}} H_{p^{\lambda}}\left(p^{l}\right)\left(\frac{p^{\lambda}}{c^{\prime}}\right)\right) .
\end{aligned}
$$

Repeating this argument for every prime $p$, it follows that the series factors as

$$
\prod_{p \neq 2}\left(\sum_{\lambda=0}^{\infty} \frac{t_{p^{\lambda}}}{p^{\lambda s}} H_{p^{\lambda}}\left(p^{v_{p}(c)}\right)\left(\frac{p^{\lambda}}{c / p^{v_{p}(c)}}\right) \chi\left(p^{\lambda}\right)\right) .
$$

We now compute the local factors of (2-12), i.e., we compute, for $p \neq 2$,

$$
\sum_{\lambda=0}^{\infty} \frac{t_{p^{\lambda}} \chi\left(p^{\lambda}\right)}{p^{\lambda s}} H_{p^{\lambda}}\left(p^{l}\right)\left(\frac{p^{\lambda}}{c^{\prime}}\right)
$$

where $l=v_{p}(c)$ and $c^{\prime}=c / p^{v_{p}(c)}$. If $l=0$ the sum reduces to

$$
\sum_{\lambda=0}^{\infty} \frac{t_{p^{\lambda}} \chi\left(p^{\lambda}\right)}{p^{\lambda s}}\left(\frac{p^{\lambda}}{c}\right)=L^{(p)}\left(s, \psi \otimes \tilde{\chi}_{c_{0}} \chi\right),
$$

where we have used that $\tilde{\chi}_{c}(p)=\tilde{\chi}_{c_{0}}(p)$ if $(p, c)=1$. Here $c_{0}$ denotes the squarefree part of $c$.

If $l>0$ is even we use Proposition 2.1 (3) to see that in this case (2-13) is equal to

$$
\left(-\frac{t_{p^{l-1}} p^{l-1} \chi\left(p^{l-1}\right)}{p^{(l-1) s}}\left(\frac{p^{l-1}}{c^{\prime}}\right)+\sum_{\lambda=l}^{\infty} \frac{t_{p^{\lambda}} p^{l-1}(p-1) \chi\left(p^{\lambda}\right)}{p^{\lambda s}}\left(\frac{p^{\lambda}}{c^{\prime}}\right)\right) .
$$


For $t_{n}$ being a Hecke eigenvalue we can use the Satake parameters and evaluate the resulting geometric sums to see that

$$
\begin{aligned}
& \sum_{\lambda=l}^{\infty} \frac{t_{p^{\lambda}} \chi\left(p^{\lambda}\right)}{p^{\lambda s}}\left(\frac{p^{\lambda}}{c^{\prime}}\right) \\
& =\frac{1}{\alpha_{p}-\beta_{p}} \sum_{\lambda=l}^{\infty} \frac{\alpha_{p}^{\lambda+1}-\beta_{p}^{\lambda+1}}{p^{\lambda s}} \chi\left(p^{\lambda}\right)\left(\frac{p^{\lambda}}{c^{\prime}}\right) \\
& =\frac{1}{\alpha_{p}-\beta_{p}}\left(\frac{\alpha_{p}^{l+1}}{p^{l s}}\left(1-\alpha_{p}\left(\frac{p}{c^{\prime}}\right) \chi(p) p^{-s}\right)^{-1}-\frac{\beta_{p}^{l+1}}{p^{l s}}\left(1-\beta_{p}\left(\frac{p}{c^{\prime}}\right) \chi(p) p^{-s}\right)^{-1}\right) \\
& =\frac{L^{(p)}\left(s, \psi \otimes \tilde{\chi}_{c_{0}} \chi\right)}{p^{l s}} \quad\left(\text { where we have used }\left(\left(\frac{p}{c^{\prime}}\right) \chi(p)\right)^{l}=1\right), \\
& =\frac{1}{\alpha_{p}-\beta_{p}}\left(\alpha_{p}^{l+1}\left(1-\beta_{p}\left(\frac{p}{c^{\prime}}\right) \chi(p) p^{-s}\right)-\beta_{p}^{l+1}\left(1-\alpha_{p}\left(\frac{p}{c^{\prime}}\right) \chi(p) p^{-s}\right)\right) \\
& \left.p^{l s} \tilde{\chi}_{c_{0}} \chi\right)
\end{aligned}
$$

This is also true when $t_{n}=\tau(n)$ by a similar computation, which we omit.

It follows that (2-14) can be written as

$$
\begin{aligned}
& p^{l-1}\left[\frac{-t_{p^{l-1}}}{p^{(l-1) s}} \tilde{\chi}_{c_{0}}\left(p^{l-1}\right) \chi\left(p^{l-1}\right)\right. \\
& \left.+\frac{L^{(p)}\left(s, \psi \otimes \tilde{\chi}_{c_{0}} \chi\right)}{p^{l s}}(p-1)\left(t_{p^{l}}-t_{p^{l-1}}\left(\frac{p}{c^{\prime}}\right) \chi(p) p^{-s}\right)\right] \\
& =p^{l-1} \frac{L^{(p)}\left(s, \psi \otimes \tilde{\chi}_{c_{0}} \chi\right)}{p^{l s}}\left[\frac{-t_{p^{l-1}}}{p^{-s}} \tilde{\chi}_{c_{0}}\left(p^{l-1}\right) \chi\left(p^{l-1}\right)\left(1-t_{p} \tilde{\chi}_{c_{0}}(p) \chi(p) p^{-s}+p^{-2 s}\right)\right. \\
& \left.+(p-1)\left(t_{p^{l}-t_{p^{l-1}}}\left(\frac{p}{c^{\prime}}\right) \chi(p) p^{-s}\right)\right] \\
& =p^{l / 2} \frac{L^{(p)}\left(s, \psi \otimes \tilde{\chi}_{c_{0}} \chi\right)}{p^{l\left(s-\frac{1}{2}\right)+1}}\left[p t_{p^{l}}-t_{p^{l-1}} \tilde{\chi}_{c_{0}}(p) \chi(p)\left(p^{1-s}+p^{s}\right)+t_{p^{l-2}}\right],
\end{aligned}
$$

using that the Hecke-eigenvalues satisfy $t_{p^{l-1}} t_{p}=t_{p^{l}}+t_{p^{l-2}}$.

If instead $l>0$ is odd we can again use Proposition 2.3 (3) and we find that in this case (2-13) is equal to

$$
\frac{t_{p^{l-1}}}{p^{(l-1) s}} p^{l-\frac{1}{2}}\left(\frac{p^{l-1}}{c^{\prime}}\right) \chi\left(p^{l-1}\right)=\frac{t_{p^{l-1}}}{p^{(l-1)(s-1)-\frac{1}{2}}} .
$$


We note also that $\tilde{\chi}_{c_{0}}(p)=\left(\frac{p}{c_{0}}\right)=0$ since by $l$ being odd we may conclude that $c_{0}$ is divisible by $p$. It follows that, in this case, $L^{(p)}\left(s, \psi \otimes \tilde{\chi}_{c_{0}} \chi\right)=1$, and we conclude that (2-13) can be written as

$$
\frac{p^{l / 2} t_{p^{l-1}}}{p^{(l-1)\left(s-\frac{1}{2}\right)}} L^{(p)}\left(s, \psi \otimes \tilde{\chi}_{c_{0}} \chi\right),
$$

which gives the desired result in this case.

Proposition 2.5. The function $Q(s, c, \chi)$ has the following properties:

(1) If $c$ is squarefree, then $Q(s, c, \chi)=1$.

(2) If $c=c_{0} c_{1}^{2}$ with $c_{0}$ squarefree and $c_{0}, c_{1}$ odd, then

$$
\left(c_{1}^{2}\right)^{1-2 s} Q(1-s, c, \chi)=Q(s, c, \chi) .
$$

Proof. Statement (1) is clear and (2) is easily verified by considering factors.

We would like to have bounds analogous to Proposition 2.3 (3). Any bound of the form $\left|t_{p^{l}}\right| \leq \tau\left(p^{l}\right) p^{\theta l}$ implies that, when $\Re(s) \geq \frac{1}{2}$,

$$
|Q(s, c, \chi)| \leq \tau(c) 4^{\#\{p \mid c\}} c^{\theta}=O\left(c^{\theta+\epsilon}\right) .
$$

The Ramanujan-Petersson conjecture will give the strongest bound with $\theta=0$. Since the Ramanujan-Petersson conjecture is true on average by (2-2), we can prove that $Q(s, c, \chi)$ is bounded on average:

Lemma 2.6. For $\Re(s) \geq \frac{1}{2}$ we have

$$
\sum_{\substack{c \leq X \\ c \text { odd }}}|Q(s, c, \chi)|^{2}=O\left(X^{1+\varepsilon}\right)
$$

uniformly in s.

Proof. Write $c=c_{0} c_{1}^{2}$ with $c_{0}$ squarefree and $c$ odd. It is easy to see that

$$
\begin{aligned}
|Q(s, c, \chi)| & \leq \prod_{p \mid c_{1}}\left(\left|t_{p^{2 v_{p}\left(c_{1}\right)}}\right|+2\left|t_{p^{2 v_{p}\left(c_{1}\right)-1}}\right|+\left|t_{p^{2 v_{p}\left(c_{1}\right)-2}}\right|\right) \\
& \leq \prod_{p \mid c_{1}} 4 \max _{i=0,1,2}\left|t_{p^{2 v_{p}\left(c_{1}\right)-i} \mid}\right| \\
& =4^{\#\left\{p \mid c_{1}\right\}}\left|t_{d_{0}}\right|, \quad \text { where } d_{0} \text { is some divisor of } c_{1}^{2} .
\end{aligned}
$$

It follows that

$$
|Q(s, c, \chi)|^{2} \leq 16^{\#\left\{p \mid c_{1}\right\}}\left|t_{d_{0}}\right|^{2} \leq 16^{\#\{p \mid c\}} \sum_{d \mid c}\left|t_{d}\right|^{2} .
$$


Using the Ramanujan-Petersson conjecture on average, $(2-2)$, and $16^{\#\{p \mid c\}}=O\left(c^{\varepsilon}\right)$, we find

$$
\begin{aligned}
\sum_{c \leq X}|Q(s, c, \chi)|^{2} & =O\left(X^{\varepsilon} \sum_{c \leq X} \sum_{d \mid c}\left|t_{d}\right|^{2}\right) \\
& =O\left(X^{\varepsilon} \sum_{d \leq X}\left|t_{d}\right|^{2} \#\{c \leq X \mid d \text { divides } c\}\right) \\
& =O\left(X^{1+\varepsilon} \sum_{d \leq X} \frac{\left|t_{d}\right|^{2}}{d}\right)=O\left(X^{1+\varepsilon}\right) .
\end{aligned}
$$

We are now ready to define the double Dirichlet series. Let $\chi_{4}$ be the primitive character $\bmod 4, \chi_{4}(n)=\left(\frac{-1}{n}\right)=(-1)^{(n-1) / 2}$ for $(n, 2)=1$, and let $\chi_{8}$ be the primitive character mod 8 given by $\chi_{8}(n)=\left(\frac{2}{n}\right)=(-1)^{\frac{1}{8}(n-1)(n+1)}$ for $(n, 2)=1$. Let $\chi, \chi^{\prime}$ be characters mod 8 , i.e., $\chi, \chi^{\prime}$ are induced from $1, \chi_{4}, \chi_{8}$, or $\chi_{4} \chi_{8}$. We then define

$$
Z\left(s, w, \chi, \chi^{\prime}\right)=\zeta_{2}(4 s-1) \sum_{\substack{n=1 \\(n, 2)=1}}^{\infty} \frac{\chi(n) t_{n} L^{*}\left(2 w-\frac{1}{2}, n, \chi^{\prime}\right)}{n^{s-w+\frac{1}{2}}} .
$$

It is easy to see - using Proposition 2.3 (3) and (2-8) - that for $\Re\left(2 w-\frac{1}{2}\right)$, $\Re\left(s-w+\frac{1}{2}\right)$ large enough the series is absolutely and locally uniformly convergent.

By Lemma 2.2 we see that

$$
Z\left(s, w, \chi, \chi^{\prime}\right)=\zeta_{2}(4 s-1) \zeta_{2}(4 w-1) \sum_{\substack{n=1 \\(n, 2)=1}}^{\infty} \frac{t_{n} \chi(n)}{n^{s-w+\frac{1}{2}}} \sum_{\substack{c=1 \\(c, 2)=1}}^{\infty} \frac{\chi^{\prime}(c) H_{n}(c)}{c^{2 w}} .
$$

Interchanging summations and using Lemma 2.4 we see that this equals

$$
Z\left(s, w, \chi, \chi^{\prime}\right)=\zeta_{2}(4 s-1) \zeta_{2}(4 w-1) \sum_{\substack{c=1 \\(c, 2)=1}}^{\infty} \frac{\chi^{\prime}(c) L^{*}\left(s-w+\frac{1}{2}, c, \psi, \chi\right)}{c^{2 w-\frac{1}{2}}}
$$

Note that, since

$$
\zeta_{2}(4 s-1) \zeta_{2}(4 w-1)=\sum_{\substack{n=1 \\(n, 2)=1}}^{\infty} \frac{\sigma_{2-4\left(s-w+\frac{1}{2}\right)}(n)}{n^{2\left(2 w-\frac{1}{2}\right)}},
$$

we also have the series representation

$$
Z\left(s, w, \chi, \chi^{\prime}\right)=\sum_{\substack{c=1 \\(c, 2)=1}}^{\infty} \frac{\chi^{\prime}(c) L^{* *}\left(s-w+\frac{1}{2}, \psi, c, \chi\right)}{c^{2 w-\frac{1}{2}}},
$$


where

$$
L^{* *}(s, \psi, c, \chi)=Q^{*}(s, c, \chi) L_{2}\left(s, \psi \otimes \tilde{\chi}_{c_{0}} \chi\right)
$$

with

$$
Q^{*}(s, c, \chi)=\sum_{l^{2} \mid c} \sigma_{2-4 s}(l) Q\left(s, c / l^{2}, \chi\right) .
$$

Remark 2.7. The two representations (2-17), (2-18) will be instrumental in proving meromorphic continuation of $Z\left(s, w, \chi, \chi^{\prime}\right)$ to $\mathbb{C}^{2}$. The proof follows the strategy outlined in [Bump et al. 1996; Diaconu et al. 2003]. The choice of arguments in the definition of (2-17), $2 w-\frac{1}{2}$ and $s-w+\frac{1}{2}$, might seem a bit strange, but for the purpose we have in mind it is the most natural one. We shall see that with this choice the functional equations are especially simple.

2B. Functional equations of the standard L-functions. We now recall the functional equations for the two $L$-functions $L\left(s, \chi_{n_{0}} \chi\right)$ and $L\left(s, \psi \otimes \tilde{\chi}_{c_{0}} \chi\right)$.

2B1. $\mathrm{GL}_{1}$. We will use the functional equation for $L_{2}\left(s, \chi_{n_{0}} \chi\right)$ for $n_{0}$ a squarefree odd natural number, and $\chi \bmod 8$ : Let $\chi_{0}^{8}$ be the trivial character mod 8 . We have that $\chi_{n_{0}} \chi$ is odd precisely if $\chi=\chi_{4} \chi_{0}^{8}$ or $\chi=\chi_{4} \chi_{8}$. Also it is known (see [Davenport 2000, Chapter 5], for example) that $\chi_{n_{0}} \chi$ is induced from the primitive character

It follows that

$$
\left(\chi_{n_{0}} \chi\right)^{*}= \begin{cases}\chi_{n_{0}} & \text { if } n_{0} \equiv 1(\bmod 4), \chi=\chi_{0}^{8}, \\ \chi_{4} \chi_{-n_{0}} & \text { if } n_{0} \neq 1(\bmod 4), \chi=\chi_{0}^{8}, \\ \chi_{4} \chi_{n_{0}} & \text { if } n_{0} \equiv 1(\bmod 4), \chi=\chi_{4} \chi_{0}^{8}, \\ \chi_{-n_{0}} & \text { if } n_{0} \neq 1(\bmod 4), \chi=\chi_{4} \chi_{0}^{8}, \\ \chi_{8} \chi_{n_{0}} & \text { if } n_{0} \equiv 1(\bmod 4), \chi=\chi_{8} \chi_{0}^{8}, \\ \chi_{4} \chi_{8} \chi_{-n_{0}} & \text { if } n_{0} \neq \equiv 1(\bmod 4), \chi=\chi_{8} \chi_{0}^{8}, \\ \chi_{4} \chi_{8} \chi_{n_{0}} & \text { if } n_{0} \equiv 1(\bmod 4), \chi=\chi_{4} \chi_{8} \chi_{0}^{8}, \\ \chi_{8} \chi_{-n_{0}} & \text { if } n_{0} \neq 1(\bmod 4), \chi=\chi_{4} \chi_{8} \chi_{0}^{8} .\end{cases}
$$

$$
L\left(s,\left(\chi_{n_{0}} \chi\right)^{*}\right)=\left(\frac{\delta_{n_{0}, \chi}}{\pi}\right)^{\frac{1}{2}-s} \frac{\Gamma\left(\frac{1}{2}\left(1-s+\kappa_{\chi}\right)\right)}{\Gamma\left(\frac{1}{2}\left(s+\kappa_{\chi}\right)\right)} L\left(1-s,\left(\chi_{n_{0}} \chi\right)^{*}\right),
$$

where

$$
\begin{aligned}
\kappa_{\chi} & = \begin{cases}0 & \text { if } \chi=\chi_{0}^{8}, \chi_{8}, \\
1 & \text { if } \chi=\chi_{4} \chi_{0}^{8}, \chi_{4} \chi_{8}\end{cases} \\
\delta_{n_{0}, \chi} & = \begin{cases}n_{0} & \text { if } \chi=\chi_{0}^{8}, n_{0} \equiv 1(\bmod 4) \text { or } \chi=\chi_{4} \chi_{0}^{8}, n_{0} \neq 1(\bmod 4), \\
4 n_{0} & \text { if } \chi=\chi_{0}^{8}, n_{0} \neq \equiv 1(\bmod 4) \text { or } \chi=\chi_{4} \chi_{0}^{8}, n_{0} \equiv 1(\bmod 4), \\
8 n_{0} & \text { if } \chi=\chi_{8}, \chi_{4} \chi_{8} .\end{cases}
\end{aligned}
$$

Note that all the functional equations are even, i.e., $\frac{G_{1}\left(\left(\chi_{n_{0}} \chi\right)^{*}\right)}{i^{\kappa_{\chi}} \sqrt{\delta_{n_{0}, \chi}}}=1$. 
We have

$$
L\left(s, \chi_{n_{0}} \chi\right)=\prod_{p \mid 8 n_{0} / \delta_{n_{0}, \chi}}\left(1-\left(\chi_{n_{0}} \chi\right)^{*}(p) p^{-s}\right) L\left(s,\left(\chi_{n_{0}} \chi\right)^{*}\right)
$$

and also

$$
L_{2}\left(s, \chi_{n_{0}} \chi\right)=L_{2}\left(s,\left(\chi_{n_{0}} \chi\right)^{*}\right)=L\left(s,\left(\chi_{n_{0}} \chi\right)^{*}\right) h_{2}\left(s, n_{0}, \chi\right),
$$

where $h_{2}\left(s, n_{0}, \chi\right)$ is either $1,1-2^{-s}$, or $1+2^{-s}$. Since $\left(\chi_{n_{0}} \chi\right)^{*}(2)$ depends only on $\chi$ and $n_{0} \bmod 8, h_{2}$ has the same dependence.

2B2. GL $L_{2}$. We now turn to $L_{2}\left(s, \psi \otimes \tilde{\chi}_{c_{0}} \chi\right)$ for $c_{0}$ a squarefree odd natural number, and $\chi \bmod 8$. The character $\tilde{\chi}_{c_{0}}$ is primitive of conductor $c_{0}$, and is even precisely when $\tilde{\chi}_{c_{0}}(-1)=\chi_{4}\left(c_{0}\right)=1$, i.e., when $c_{0} \equiv 1(\bmod 4)$. A reference on twisting of automorphic forms (at least for modular forms) is [Iwaniec and Kowalski 2004, Section 14.8].

We need to take special care of 2-factors. For any primitive automorphic form $f$ for $\mathrm{GL}_{2}$ we define a polynomial $p_{2, f}(z)$ of degree 1 or 2 , depending on whether 2 is ramified or not, by

$$
\frac{1}{p_{2, f}(z)}=\sum_{j=0}^{\infty} t_{2^{j}}(f) z^{j}
$$

where $t_{n}(f)$ are the coefficients of $L(s, f)$. In particular the 2-factor of $L(s, f)$ equals $p_{2, f}^{-1}\left(2^{-s}\right)$. If $p_{2, f}$ is of degree $2, p_{2, f}(z)=\left(1-\alpha_{2} z\right)\left(1-\beta_{2} z\right)$, the estimate $\left|\alpha_{2}\right|,\left|\beta_{2}\right|<2^{1 / 5}$ [Shahidi 1988, p. 549] shows that $p_{2, f}\left( \pm 2^{-s}\right)$ is uniformly bounded away from 0 at $\Re(s) \geq \frac{1}{2}$. If $p_{2, f}(z)$ is of degree 1 , the explicit value of $t_{2}(=0$ or $\pm 1 / \sqrt{2})$ shows that $p_{2, f}\left( \pm 2^{-s}\right)$ does not vanish on $\Re(s) \geq \frac{1}{2}$ and, as a result,

$$
\frac{1}{p_{2, f}\left( \pm 2^{-s}\right)}=O(1)
$$

uniformly in $f$ when $\Re(s) \geq \frac{1}{2}$.

We assume now that $\psi$ is primitive Maaß Hecke form for $\Gamma_{0}(4)$ with real Fourier coefficients. The twisted function $\psi \otimes \chi$ is still a Hecke form with trivial character $\chi^{2}$ but not necessarily primitive. Let $g=(\psi \otimes \chi)^{*}$ be the primitive form whose Fourier coefficients agree with those of $\psi \otimes \chi$ except possibly at the 2-factor. This is a cusp form of level $N=N_{\psi, \chi}=2^{j}$, a divisor of 64. For fixed $\psi$ there are 4 such forms $g$, as there are 4 characters mod 8 . We have that $L_{2}(s, \psi \otimes \chi)=L_{2}(s, g)$ since the Fourier coefficients of $g$ and $\psi \otimes \chi$ agree on odd numbers.

We now twist $g$ by $\tilde{\chi}_{c_{0}}$. Since the conductor of $\tilde{\chi}_{c_{0}}$ is relatively prime to the level of $g$, the result is a primitive cusp form of level $N \cdot c_{0}^{2}$. The twisted $L$-function 
$L\left(s, \psi \otimes \tilde{\chi}_{c_{0}} \chi\right)$ agrees with $L\left(s, g \otimes \tilde{\chi}_{c_{0}}\right)$ outside the prime 2 , so that

$$
L_{2}\left(s, g \otimes \tilde{\chi}_{c_{0}}\right)=L_{2}\left(s, \psi \otimes \tilde{\chi}_{c_{0}} \chi\right) .
$$

We have the functional equation of $g \otimes \tilde{\chi}_{c_{0}}$ :

$L\left(s, g \otimes \tilde{\chi}_{c_{0}}\right)$

$=\epsilon\left(g, \tilde{\chi}_{c_{0}}\right)\left(\frac{N c_{0}^{2}}{\pi^{2}}\right)^{\frac{1}{2}-s} \prod_{\epsilon \in\{ \pm 1\}} \frac{\Gamma\left(\frac{1}{2}\left(1-s+\kappa_{\chi}, \psi, c_{0}+\epsilon\left(s_{0}-\frac{1}{2}\right)\right)\right)}{\Gamma\left(\frac{1}{2}\left(s+\kappa_{\chi}, \psi, c_{0}+\epsilon\left(s_{0}-\frac{1}{2}\right)\right)\right)} L\left(1-s, g \otimes \tilde{\chi}_{c_{0}}\right)$.

This functional equation involves the root number $\epsilon\left(g, \tilde{\chi}_{c_{0}}\right)$ that depends on $c_{0}$ mod 8 , as it is given by

$$
\epsilon(g) \chi^{2}\left(c_{0}\right) \tilde{\chi}_{c_{0}}\left(2^{j}\right) G\left(\tilde{\chi}_{c_{0}}\right)^{2} / c_{0},
$$

where $\epsilon(g)$ is the root number of $g$. We have

$$
L_{2}\left(s, \psi \otimes \tilde{\chi}_{c_{0}} \chi\right)=H_{2}\left(s, g, c_{0}\right) L\left(s, g \otimes \tilde{\chi}_{c_{0}}\right),
$$

where

$$
H_{2}\left(s, g, c_{0}\right)=p_{2, g \otimes \tilde{\chi}_{c_{0}}}\left(2^{-s}\right)=p_{2, g}\left(\tilde{\chi}_{c_{0}}(2) 2^{-s}\right) .
$$

The dependence of $H_{2}\left(s, g, c_{0}\right)$ on $c_{0}$ is only $\bmod 8$, as it involves $\tilde{\chi}_{c_{0}}(2)$. We note also that $\kappa_{\chi, \psi, c_{0}}=\kappa_{\chi}, \psi \tilde{\chi}_{c_{0}}(-1)$ depends only on $c_{0} \bmod 4$ since $\tilde{\chi}_{c_{0}}(-1)=\chi_{4}\left(c_{0}\right)$.

Remark 2.8. In the $G L_{1} \times G L_{1}$ case, i.e., if $\psi=\psi_{\tau}$ and $t_{n}=\tau(n)$, we have

$$
L\left(s, \psi_{\tau} \otimes \tilde{\chi}_{c_{0}} \chi\right):=\sum_{n=1}^{\infty} \frac{\tau(n) \tilde{\chi}_{c_{0}} \chi(n)}{n^{s}}=L\left(s, \tilde{\chi}_{c_{0}} \chi\right)^{2} .
$$

We see (after using quadratic reciprocity) that the analogues of the results of this section follow from Section 2B1.

2C. Average bounds on twisted L-functions. Before we can give the proof of the meromorphic continuation we recall a few facts concerning the involved $L$-series. We first recall an average bound on $L$-functions twisted with quadratic characters. The main ingredient in proving such a bound is Heath-Brown's large sieve estimate for quadratic characters. He proves [1995, Theorem 1] that for any positive $\varepsilon>0$ there exists a constant $C>0$ such that for any positive integers $M, N$ and for arbitrary complex numbers $a_{1}, \ldots, a_{N}$ we have

$$
\sum_{m \leq M}^{*}\left|\sum_{n \leq N}^{*} a_{n}\left(\frac{n}{m}\right)\right|^{2} \leq C(M N)^{\varepsilon}(M+N) \sum_{n \leq N}^{*}\left|a_{n}\right|^{2} .
$$

Here a $*$ means summation over positive odd squarefree integers. From this one can prove the following. 
Theorem 2.9. For $\Re(s) \geq \frac{1}{2}$,

$$
\begin{aligned}
& \sum_{\substack{1<d_{0} \leq X \\
d_{0} \text { odd, } \\
\text { squarefree }}}\left|L\left(s, \chi_{d_{0}} \chi\right)\right|^{4}=O\left((X|s|)^{1+\epsilon}\right), \\
& \sum_{\substack{1<d_{0} \leq X \\
d_{0} \text { odd, } \\
\text { squarefree }}}\left|L\left(s, \psi \otimes \tilde{\chi}_{d_{0}} \chi\right)\right|^{2}=O\left((X|s|)^{1+\epsilon}\right) .
\end{aligned}
$$

The bound (2-29) is already in [Heath-Brown 1995, Theorem 2] and (2-30) is essentially proved in the same way. See also [Soundararajan and Young 2010, Section 2.3; Chinta and Diaconu 2005, Lemma 3.2]. These bounds give the Lindelöf hypothesis on average in the character aspect, while keeping the convexity bound in the $s$ aspect when $\Re(s)=\frac{1}{2}$.

Remark 2.10. By considering 2-factors it is straightforward to see that the above bounds (2-29) and (2-30) are true also if we remove 2-factors, i.e., replace $L$ by $L_{2}$.

2D. Meromorphic continuation and functional equations of $Z\left(s, w, \chi, \chi^{\prime}\right)$. We first analyze $Z\left(s, w, \chi, \chi^{\prime}\right)$ from the representation (2-17).

Theorem 2.11. The function $\left(w-\frac{3}{4}\right) Z\left(s, w, \chi, \chi^{\prime}\right)$ is analytic in

$$
D_{1}=\left\{(s, w): \Re(s-w)>\frac{1}{2}, \mathfrak{R}(s+w)>\frac{3}{2}\right\},
$$

and satisfies a functional equation $\alpha:(s, w) \mapsto(s, 1-w)$ given by

$$
\left(1-2^{-(3-4 w)}\right) Z\left(s, w, \chi, \chi^{\prime}\right)=\frac{\Gamma\left(\frac{1}{2}\left(\frac{3}{2}-2 w+\kappa_{\chi^{\prime}}\right)\right)}{\Gamma\left(\frac{1}{2}\left(2 w-\frac{1}{2}+\kappa_{\chi^{\prime}}\right)\right)} \sum_{\chi^{\prime \prime} \bmod 8} p_{\chi^{\prime \prime}}(w) Z\left(s, 1-w, \chi^{\prime \prime}, \chi^{\prime}\right) \text {. }
$$

Here the $p_{\chi^{\prime \prime}}(w)$ are polynomials in $2^{-w}$. In particular they are bounded in vertical strips. Furthermore, away from $w=\frac{3}{4}$,

$Z\left(s, w, \chi, \chi^{\prime}\right)= \begin{cases}O\left((|w|+1)^{\frac{1}{4}+\varepsilon}\right) & \text { if } \frac{1}{2} \leq \Re w \leq K, \Re(s-w) \geq \frac{1}{2}+\delta, \\ O\left((|w|+1)^{\frac{1}{4}+1-2 \Re(w)+\varepsilon}\right) & \text { if }-K \leq \Re w \leq \frac{1}{2}, \Re(s+w) \geq \frac{3}{2}+\delta,\end{cases}$

for any fixed $K>\frac{1}{2}$ and $\delta>0$.

Remark 2.12. We shall see in the proof that the factor $\left(w-\frac{3}{4}\right)$ is only necessary when $\chi^{\prime}$ is trivial. We note also that the implied constant may depend on $\psi$. Moreover, the bounds given above are not necessarily optimal. All we need for Theorem 2.15 and Lemma 2.18 below is polynomial control.

Proof. We remark that the factor $\zeta_{2}(4 s-1)$ appearing in (2-17) does not have a pole in the region $D_{1}$. Thus we only have to study the series from (2-17) to prove the analytic properties of $\left(w-\frac{3}{4}\right) Z\left(s, w, \chi, \chi^{\prime}\right)$. 
We consider the regions where the series representation (2-17) is absolutely convergent. We consider first the sum over all nonperfect squares $\left(n \neq m^{2}\right)$.

If $\Re(w) \geq \frac{1}{2}$ (which corresponds to $\Re\left(2 w-\frac{1}{2}\right) \geq \frac{1}{2}$ ) we use (2-2), Theorem 2.9, Proposition 2.3 (3), and Cauchy-Schwarz to see that, away from $w=\frac{3}{4}$,

$$
\sum_{\substack{n \leq X \\ n \neq m^{2}}}\left|t_{n} \chi(n) q\left(2 w-\frac{1}{2}, n, \chi^{\prime}\right) L_{2}\left(2 w-\frac{1}{2}, \chi_{n_{0}} \chi^{\prime}\right)\right|=O\left(X^{1+\varepsilon}|w|^{\frac{1}{4}+\varepsilon}\right) .
$$

It follows that the nonperfect square contribution is convergent for $\Re(s-w) \geq \frac{1}{2}+\delta$ and $\Re(w) \geq \frac{1}{2}$ and that, in the region $\Re(s-w) \geq \frac{1}{2}+\delta, \Re(w) \geq \frac{1}{2}$, it is analytic and bounded by $O\left(|w|^{\frac{1}{4}+\varepsilon}\right)$.

For $\mathfrak{R}(w) \leq \frac{1}{2}$, we use Proposition 2.3 (2) and the functional equation for $L_{2}\left(2 w-\frac{1}{2}, \chi_{n_{0}} \chi^{\prime}\right)$ to see that the product $q\left(2 w-\frac{1}{2}, n, \chi^{\prime}\right) L_{2}\left(2 w-\frac{1}{2}, \chi_{n_{0}} \chi^{\prime}\right)$ equals $n^{1-2 w}\left(\frac{\delta_{n_{0}, \chi^{\prime}}}{n_{0} \pi}\right)^{1-2 w} \frac{\Gamma\left(\frac{1}{2}\left(\frac{3}{2}-2 w+\kappa_{\chi^{\prime}}\right)\right)}{\Gamma\left(\frac{1}{2}\left(2 w-\frac{1}{2}+\kappa_{\chi^{\prime}}\right)\right)} q\left(2(1-w)-\frac{1}{2}, n, \chi^{\prime}\right) L_{2}\left(2(1-w)-\frac{1}{2}, \chi_{n_{0}} \chi^{\prime}\right)$ times a factor $h_{2}\left(2 w-\frac{1}{2}, n_{0}, \chi\right) / h_{2}\left(2(1-w)-\frac{1}{2}, n_{0}, \chi\right)$, which is bounded when $\Re(w) \leq \frac{1}{2}$ (recall (2-23) for the definition of $h_{2}$ ). We notice that $\delta_{n_{0}, \chi^{\prime}} / n_{0}$ is 1,4 , or 8 , and that in bounded $w$-strips the quotient of $\Gamma$-factors is $O\left(|w|^{1-2 \Re(w)}\right)$. It follows that in bounded $w$-strips and for $\Re(w) \leq \frac{1}{2}$ we have

$$
\begin{aligned}
& \sum_{\substack{n \leq X \\
n \neq m^{2}}}\left|n^{2 w-1} t_{n} \chi(n) q\left(2 w-\frac{1}{2}, n, \chi^{\prime}\right) L_{2}\left(2 w-\frac{1}{2}, \chi_{n_{0}} \chi^{\prime}\right)\right| \\
& \quad=O\left(|w|^{1-2 \Re(w)}\right) \sum_{n \leq X}\left|t_{n} \chi(n) q\left(2(1-w)-\frac{1}{2}, n, \chi^{\prime}\right) L_{2}\left(2(1-w)-\frac{1}{2}, \chi_{n_{0}} \chi^{\prime}\right)\right| \\
& \quad=O\left(|w|^{\frac{1}{4}+1-2 \Re(w)+\epsilon} X^{1+\varepsilon}\right),
\end{aligned}
$$

where in the last line we have used the same argument as used to bound (2-31). It follows that when $\Re(s+w) \geq \frac{3}{2}+\delta, \Re(w) \leq \frac{1}{2}$ the nonsquare contribution from the series in (2-17) converges absolutely and that in this region this contribution is analytic and bounded by $O\left(|w|^{\frac{1}{4}+1-2 \Re(w)+\varepsilon}\right)$.

We next consider the sum over all perfect squares $n=m^{2}$,

$$
L_{2}\left(2 w-\frac{1}{2}, \chi^{\prime}\right) \sum_{\substack{m=1 \\(m, 2)=1}}^{\infty} \frac{t_{n} \chi(n) q\left(2 w-\frac{1}{2}, m^{2}, \chi^{\prime}\right)}{m^{2\left(s-w+\frac{1}{2}\right)}} .
$$

Using Proposition 2.3 and (2-2) we easily see that the sum is convergent in

$$
\{(s, w): \Re(s-w)>0, \Re(s+w)>1\},
$$


and that the factor in front has a simple pole at $w=\frac{3}{4}$ if $\chi^{\prime}$ is trivial. That this contribution has the desired growth properties follows from the convexity estimate on $L_{2}\left(2 w-\frac{1}{2}, \chi^{\prime}\right)$.

Having established that $\left(w-\frac{3}{4}\right) Z\left(s, w, \chi, \chi^{\prime}\right)$ is analytic in $D_{1}$, we now show that it satisfies a functional equation here. For $(s, w)$ in this region we use the functional equation (2-21) and Proposition 2.3 and the subsequent discussion to see that $Z\left(s, w, \chi, \chi^{\prime}\right) / \zeta_{2}(4 s-1)$ equals

$$
\begin{aligned}
& \sum_{\substack{n=1 \\
(n, 2)=1}}^{\infty} \frac{t_{n} \chi(n) q\left(2 w-\frac{1}{2}, n, \chi^{\prime}\right) L_{2}\left(2 w-\frac{1}{2}, \chi_{n_{0}} \chi^{\prime}\right)}{n^{s-w+\frac{1}{2}}} \\
& =\sum_{\substack{n=1 \\
(n, 2)=1}}^{\infty} n^{1-2 w}\left(\frac{\delta_{n_{0}, \chi^{\prime}}}{n_{0} \pi}\right)^{1-2 w} \frac{h_{2}\left(2 w-\frac{1}{2}, n_{0}, \chi^{\prime}\right)}{h_{2}\left(2(1-w)-\frac{1}{2}, n_{0}, \chi^{\prime}\right)} \frac{\Gamma\left(\frac{1}{2}\left(\frac{3}{2}-2 w+\kappa_{\chi^{\prime}}\right)\right)}{\Gamma\left(\frac{1}{2}\left(2 w-\frac{1}{2}+\kappa_{\chi^{\prime}}\right)\right)} \\
& \cdot \frac{t_{n} \chi(n) q\left(2(1-w)-\frac{1}{2}, n, \chi^{\prime}\right) L_{2}\left(2(1-w)-\frac{1}{2}, \chi_{n_{0}} \chi^{\prime}\right)}{n^{s-w+\frac{1}{2}}} \\
& =\pi^{2 w-1} \frac{\Gamma\left(\frac{1}{2}\left(\frac{3}{2}-2 w+\kappa_{\chi^{\prime}}\right)\right)}{\Gamma\left(\frac{1}{2}\left(2 w-\frac{1}{2}+\kappa_{\chi^{\prime}}\right)\right)} \sum_{\substack{n=1 \\
(n, 2)=1}}^{\infty} \frac{h_{2}\left(2 w-\frac{1}{2}, n_{0}, \chi^{\prime}\right)}{h_{2}\left(2(1-w)-\frac{1}{2}, n_{0}, \chi^{\prime}\right)}\left(\frac{\delta_{n_{0}, \chi^{\prime}}}{n_{0}}\right)^{1-2 w} \\
& \cdot \frac{t_{n} \chi(n) q\left(2(1-w)-\frac{1}{2}, n, \chi^{\prime}\right) L_{2}\left(2(1-w)-\frac{1}{2}, \chi_{n_{0}} \chi^{\prime}\right)}{n^{s-(1-w)+\frac{1}{2}}} .
\end{aligned}
$$

We split the sum according to $n \bmod 8$, and notice that for fixed $\chi^{\prime}$ the function

$$
\frac{h_{2}\left(2 w-\frac{1}{2}, n_{0}, \chi^{\prime}\right)}{h_{2}\left(2(1-w)-\frac{1}{2}, n_{0}, \chi^{\prime}\right)}\left(\frac{\delta_{n_{0}, \chi^{\prime}}}{n_{0}}\right)^{1-2 w}
$$

is the same fraction of Dirichlet polynomials in $2^{-w}$ throughout each of these sums, so that we can put them outside the sums. Using again that the indicator function of residue class mod 8 can be written as a linear combination of characters mod 8 (at least on the odd numbers), we arrive at the functional equation for $Z\left(s, w, \chi, \chi^{\prime}\right)$. We note that the factor $1-2^{-(3-4 w)}$ is the product of all possible $h_{2}\left(2(1-w)-\frac{1}{2}, n_{0}, \chi^{\prime}\right)$. This shows that the $p_{\chi^{\prime \prime}}(w)$ are in fact polynomials in $2^{-w}$.

We now apply the same type of analysis to the second series representation of $Z\left(s, w, \chi, \chi^{\prime}\right)$ given in (2-18). Recall from Section 2B2 that $g$ denotes $(\psi \otimes \chi)^{*}$, where $\chi$ is one of the 4 characters mod 8 . Let

$$
V(s, w)=\prod_{g} p_{2, g}\left(2^{-\left(w-s+\frac{1}{2}\right)}\right) p_{2, g}\left(-2^{-\left(w-s+\frac{1}{2}\right)}\right),
$$

where $p_{2, g}(z)$ is as in $(2-24)$. 
Theorem 2.13. The function $\left(s-w-\frac{1}{2}\right)^{2} Z\left(s, w, \chi, \chi^{\prime}\right)$ is analytic in

$$
D_{2}=\left\{(s, w): \Re(s)>\frac{3}{4}, \Re(w)>\frac{3}{4}\right\},
$$

and satisfies a functional equation $\beta:(s, w) \mapsto(w, s)$ given by $V(s, w) Z\left(s, w, \chi, \chi^{\prime}\right)$

$$
=\sum_{\substack{k=0,1 \\ \chi^{\prime \prime} \bmod 8}} \prod_{\epsilon \in\{ \pm 1\}} \frac{\Gamma\left(\frac{1}{2}\left(1-\left(s-w+\frac{1}{2}\right)+k+\epsilon\left(s_{0}-\frac{1}{2}\right)\right)\right)}{\Gamma\left(\frac{1}{2}\left(\left(s-w+\frac{1}{2}\right)+k+\epsilon\left(s_{0}-\frac{1}{2}\right)\right)\right)} P_{\psi, \chi, \chi^{\prime \prime}}(s, w) Z\left(w, s, \chi, \chi^{\prime \prime}\right) .
$$

Here the $P_{\psi, \chi, \chi^{\prime \prime}}(s, w)$ are polynomials in $2^{-(s-w)}$. In particular they are functions bounded in vertical strips. Furthermore, away from $s-w-\frac{1}{2}=0$, $Z\left(s, w, \chi, \chi^{\prime}\right)= \begin{cases}O\left((|s-w|+1)^{\frac{1}{2}+\varepsilon}\right) & \text { for } \frac{3}{4}+\delta \leq \Re w \leq \Re(s) \leq K, \\ O\left((|s-w|+1)^{\frac{3}{2}-2 \Re\left(s-w+\frac{1}{2}+\varepsilon\right)}\right) & \text { for } \frac{3}{4}+\delta \leq \Re s \leq \mathfrak{R}(w) \leq K,\end{cases}$ where $K, \delta$ are any constants with $K>\frac{3}{4}$ and $\delta>0$.

Remark 2.14. We shall see in the proof that the factor $\left(s-w-\frac{1}{2}\right)^{2}$ is only necessary when $\psi$ is $\mathrm{GL}_{1} \times \mathrm{GL}_{1}$ and $\chi$ is trivial. We note also that the implied constant may depend on $\psi$. Moreover, as before the bounds given above are not necessarily optimal, as all we need for Theorem 2.15 and Lemma 2.18 below is polynomial control.

Proof. We now want to find the region of absolute convergence of (2-18). Consider first the region $\Re\left(s-w+\frac{1}{2}\right) \geq \frac{1}{2}$. We can use Cauchy-Schwarz, Theorem 2.9, and Lemma 2.6 to see that the sum over nonperfect squares satisfies

$$
\sum_{\substack{c \leq X \\ c \neq r^{2}, c \text { odd }}}\left|\chi^{\prime}(c) Q\left(s-w+\frac{1}{2}, c, \chi\right) L_{2}\left(s-w+\frac{1}{2}, \psi \otimes \tilde{\chi}_{c_{0}} \chi\right)\right|=O\left(X^{1+\varepsilon}(1+|s-w|)^{\frac{1}{2}+\varepsilon}\right) .
$$

Hence the sum over these terms is absolutely convergent when $\Re\left(2 w-\frac{1}{2}\right) \geq 1+\delta$. The sum over the perfect squares potentially has a double pole at $s-w+\frac{1}{2}=1$ : For $t_{n}=\tau(n)$ we have $L_{2}\left(s, \psi \otimes \chi_{0}^{8}\right)=\zeta_{2}^{2}(s)$. The sum over perfect squares is

$$
L_{2}\left(s-w+\frac{1}{2}, \psi \otimes \chi\right) \sum_{\substack{c=1 \\ c=r^{2}}}^{\infty} \frac{\chi^{\prime}(c) Q\left(s-w+\frac{1}{2}, c, \chi\right)}{c^{2 w-\frac{1}{2}}},
$$

where the sum is again absolutely convergent for $\Re\left(2 w-\frac{1}{2}\right) \geq 1+\delta$, using CauchySchwarz and Lemma 2.6. It follows that, when $\Re\left(s-w+\frac{1}{2}\right) \geq \frac{1}{2}$, the sums are convergent for $\Re(w) \geq \frac{3}{4}+\delta$, and hence $Z\left(s, w, \chi, \chi^{\prime}\right)$ is analytic in this region except for a potential double polar line at $s-w+\frac{1}{2}=1$. We also find that in this region we have the bound $Z\left(s, w, \chi, \chi^{\prime}\right)=O\left((1+|s-w|)^{\frac{1}{2}+\varepsilon}\right)$. 
Turning now to $\Re\left(s-w+\frac{1}{2}\right) \leq \frac{1}{2}$, we use the functional equation (2-26) and Proposition 2.5 (2) to move to a region where we can use the same bounds as for $\Re\left(s-w+\frac{1}{2}\right) \geq \frac{1}{2}$ :

$$
\begin{aligned}
& \frac{Z\left(s, w, \chi, \chi^{\prime}\right)}{\zeta_{2}(4 s-1) \zeta_{2}(4 w-1)} \\
& =\sum_{\substack{c=1 \\
(c, 2)=1}}^{\infty} \frac{1}{c^{2 w-\frac{1}{2}}} \chi^{\prime}(c) Q\left(s-w+\frac{1}{2}, c, \chi\right) L_{2}\left(s-w+\frac{1}{2}, \psi \otimes \tilde{\chi}_{c_{0}} \chi\right) \\
& =\sum_{\substack{c=1 \\
(c, 2)=1}}^{\infty} \frac{1}{c^{2 w-\frac{1}{2}}} \chi^{\prime}(c) c^{-2(s-w)} Q\left(1-\left(s-w+\frac{1}{2}\right), c, \chi\right) \epsilon\left(\psi, \tilde{\chi}_{c_{0}} \chi\right)\left(\frac{N_{1}}{\pi^{2}}\right)^{-(s-w)} \\
& \quad \times \frac{\Gamma\left(\frac{1}{2}\left(1-\left(s-w+\frac{1}{2}\right)+\kappa_{\chi}, \psi, c_{0}+\left(s_{0}-\frac{1}{2}\right)\right)\right) \Gamma\left(\frac{1}{2}\left(1-\left(s-w+\frac{1}{2}\right)+\kappa_{\chi}, \psi, c_{0}-\left(s_{0}-\frac{1}{2}\right)\right)\right)}{\Gamma\left(\frac{1}{2}\left(\left(s-w+\frac{1}{2}\right)+\kappa_{\chi}, \psi, c_{0}+\left(s_{0}-\frac{1}{2}\right)\right)\right) \Gamma\left(\frac{1}{2}\left(\left(s-w+\frac{1}{2}\right)+\kappa_{\chi}, \psi, c_{0}-\left(s_{0}-\frac{1}{2}\right)\right)\right)} \\
& \quad \times \frac{H_{2}\left(s-w+\frac{1}{2}, g_{1}, c_{0}\right)}{H_{2}\left(1-\left(s-w+\frac{1}{2}\right), g_{1}, c_{0}\right)} L_{2}\left(1-\left(s-w+\frac{1}{2}\right), \psi \otimes \tilde{\chi}_{c_{0}} \chi\right), \quad(2-32)
\end{aligned}
$$

where $g_{1}=\left(\psi \otimes \tilde{\chi}_{c_{0}} \chi\right)^{*}$ with level $N_{1} c_{0}^{2}$ for $N_{1}$ a divisor of 64 depending on $\chi, \psi$ (recall (2-27) for the definition of $H_{2}$ ). Using the same trick as before of splitting the sum into perfect squares and nonperfect squares, and using the bounds from Lemma 2.6 and Theorem 2.9 as well as the Stirling bound on the Gamma factors and a trivial bound on the 2 -factors, we find that $Z\left(s, w, \chi, \chi^{\prime}\right)$ is analytic in

$$
\left\{(s, w): \Re\left(s-w+\frac{1}{2}\right) \leq \frac{1}{2}, \Re s \geq \frac{3}{4}+\delta\right\}
$$

and bounded, as $Z\left(s, w, \chi, \chi^{\prime}\right)=O\left(1+|s-w|^{\frac{1}{2}+\varepsilon}|s-w|^{1-2 \Re\left(s-w+\frac{1}{2}\right)}\right)$ for $\Re(s)$, $\Re(w)$ bounded in this region.

We have established that $Z\left(s, w, \chi, \chi^{\prime}\right)$ is analytic in $D_{2}$. We now show that it also satisfies a functional equation in this region. Consider (2-32). We noticed that $\epsilon\left(\psi, \tilde{\chi}_{0} \chi\right), \kappa_{\chi, \psi, c_{0}}$, and $H_{2}\left(s, g_{1}, c_{0}\right)$ depend only on $c_{0}$ modulo 8 (see Section 2B2). We split the sum into residue classes modulo 8 and we can put these data outside the sum. Since $H_{2}\left(1-\left(s-w+\frac{1}{2}\right), g_{1}, c_{0}\right)$ can have zeros in the region we multiply the left-hand side with all possible expressions of it, which is $V(s, w)$, and arrive at the desired functional equation.

Using the two previous theorems we can now show that $Z\left(s, w, \chi, \chi^{\prime}\right)$ admits a meromorphic continuation to all of $\mathbb{C}^{2}$.

Theorem 2.15. The function

$$
Z^{*}\left(s, w, \chi, \chi^{\prime}\right)=\left(s-w-\frac{1}{2}\right)^{2}\left(s+w-\frac{3}{2}\right)^{2}\left(w-\frac{3}{4}\right)\left(s-\frac{3}{4}\right) Z\left(s, w, \chi, \chi^{\prime}\right)
$$




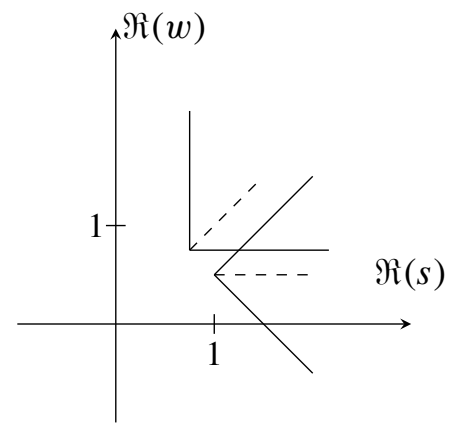

Figure 1. $D_{1} \cup D_{2}$.

admits an analytic continuation to $(s, w) \in \mathbb{C}^{2}$ with at most polynomial growth for $\Re(s), \Re(w)$ in bounded regions.

Proof. We use repeatedly the functional equations in Theorems 2.11 and 2.13 . We notice that these two theorems show that $Z^{*}\left(s, w, \chi, \chi^{\prime}\right)$ is analytic in the union of the two overlapping sets

$$
\begin{aligned}
& D_{1}=\left\{(s, w): \Re(s-w)>\frac{1}{2}, \Re(s+w)>\frac{3}{2}\right\}, \\
& D_{2}=\left\{(s, w): \Re(s)>\frac{3}{4}, \Re(w)>\frac{3}{4}\right\},
\end{aligned}
$$

since $\left(w-\frac{3}{4}\right) Z\left(s, w, \chi, \chi^{\prime}\right)$ is analytic in $D_{1}$ and $\left(s-w-\frac{1}{2}\right)^{2} Z\left(s, w, \chi, \chi^{\prime}\right)$ is analytic in $D_{2}$. (See Figure 1.)

We now use the group of functional equations generated by the two functional equations

$$
\alpha:(s, w) \mapsto(s, 1-w), \quad \beta:(s, w) \mapsto(w, s) .
$$

They generate a group of order 8 isomorphic to the dihedral group $D_{4}$ of order 8 . We note that $\alpha^{2}=\beta^{2}=\mathrm{Id}$. Using $\beta$, we see that $\left(s-\frac{3}{4}\right) Z\left(s, w, \chi, \chi^{\prime}\right)$ is a holomorphic function of at most bounded polynomial growth (bounding the ratio of Gamma functions using Stirling asymptotics) in $D_{3}=\beta D_{1}$, which then extends $Z^{*}\left(s, w, \chi, \chi^{\prime}\right)$ to $D_{1} \cup D_{2} \cup D_{3}$. We notice that the Gamma factor on the right-hand side of the functional equation in Theorem 2.13 and $V(s, w)^{-1}$ does not have poles when $\Re(w-s)>0$ (by (2-25) and properties of the Gamma function).

Using $\alpha$, we extend $Z^{*}\left(s, w, \chi, \chi^{\prime}\right)$ analytically to $D_{1} \cup D_{2} \cup \beta D_{1} \cup \alpha D_{2} \cup \alpha \beta D_{1}$. We notice that the 2 factor $\left(1-2^{-(3-4 w)}\right)^{-1}$ and the Gamma factor in Theorem 2.11 are analytic when $\Re(w)<\frac{3}{4}$. The reflection $\alpha$ of the double polar line $s-w=\frac{1}{2}$ in $D_{2}$ produces the double polar line $s+w=\frac{3}{2}$ in $\alpha D_{2}$. 

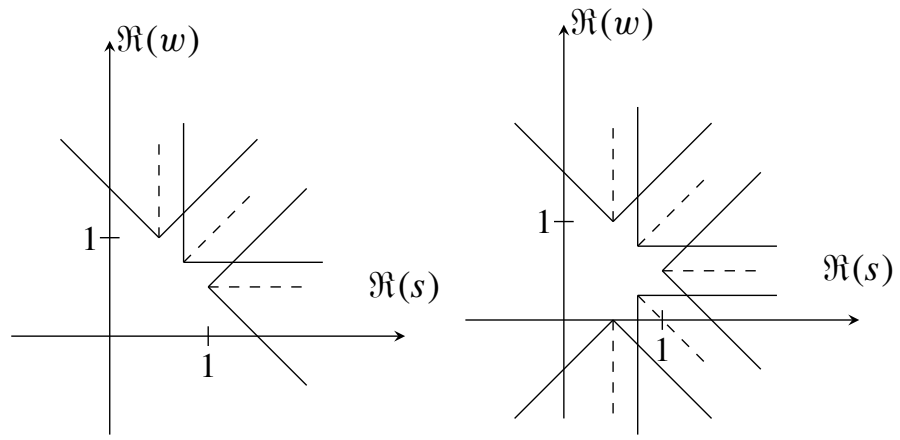

The regions $D_{4}=\beta \alpha D_{2}, D_{5}=\beta \alpha \beta D_{1}$, and $D_{6}=\alpha \beta \alpha D_{2}=\alpha D_{4}$ can be dealt with using Theorems 2.11 and 2.13 in the same way and no new polar lines are introduced, neither due to the 2 factors, nor the Gamma factors.

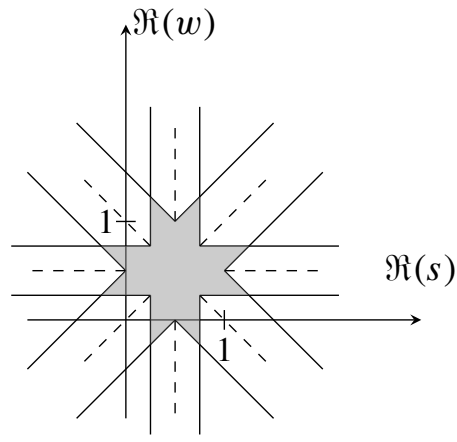

The function in (2-33) is now extended to a holomorphic function on the complement of the domain with tube given by the shaded region. It is bounded polynomially for $\Re(w), \Re(s)$ bounded. We can therefore use Bochner's tube theorem (see [Diaconu et al. 2003, Propositions 4.6 and 4.7 and the argument on p. 341]) to extend the holomorphic function to the convex hull of this region (which is $\mathbb{C}^{2}$ ) with at most polynomial bounds for $(\Re(s), \Re(w))$ in compact sets. Therefore, $Z\left(s, w, \chi, \chi^{\prime}\right)$ has the same properties, apart from being meromorphic with the specified polar lines in (2-33).

Remark 2.16. Combining Theorems 2.11 and 2.13 we note that $\alpha \circ \beta \circ \alpha \circ \beta(s, w)=$ $(1-s, 1-w)$, and it follows that there exist functions $\alpha_{\rho, \rho^{\prime}, \chi, \chi^{\prime}}(s, w)$ bounded in vertical strips such that

$$
\begin{aligned}
& F(s, w) Z\left(s, w, \chi, \chi^{\prime}\right) \\
& =\sum_{\bar{k} \in\{0,1\}^{4}} \frac{G(1-s, 1-w, \bar{k})}{G(s, w, \bar{k})} \sum_{\rho, \rho^{\prime} \bmod 8} \alpha_{\bar{k}, \rho, \rho^{\prime}, \chi, \chi^{\prime}}(s, w) Z\left(1-s, 1-w, \rho, \rho^{\prime}\right),
\end{aligned}
$$


where

$$
\begin{aligned}
G(s, w, \bar{k}):= & \Gamma\left(\frac{1}{2}\left(2 w-\frac{1}{2}+k_{1}\right)\right) \prod_{\epsilon_{1} \in\{ \pm 1\}} \Gamma\left(\frac{1}{2}\left(s+w-\frac{1}{2}+k_{2}+\epsilon_{1}\left(s_{0}-\frac{1}{2}\right)\right)\right) \\
& \cdot \Gamma\left(\frac{1}{2}\left(2 s-\frac{1}{2}+k_{3}\right)\right) \prod_{\epsilon_{2} \in\{ \pm 1\}} \Gamma\left(\frac{1}{2}\left(s-w+\frac{1}{2}+k_{4}+\epsilon_{2}\left(s_{0}-\frac{1}{2}\right)\right)\right)
\end{aligned}
$$

and

$$
F(s, w):=\left(1-2^{-(3-4 w)}\right)\left(1-2^{-(3-4 s)}\right) V(s, w) V(w, 1-s) .
$$

Using $\frac{\Gamma\left(\frac{1}{2}(1-z+1)\right)}{\Gamma\left(\frac{1}{2}(z+1)\right)}=\frac{\Gamma\left(\frac{1}{2}(1-z)\right)}{\Gamma\left(\frac{1}{2} z\right)} \cot (\pi z / 2)$ we see that

$$
\frac{G(1-s, 1-w, \bar{k})}{G(s, w, \bar{k})}=\frac{G(1-s, 1-w, 0)}{G(s, w, 0)} \cot _{\bar{k}}(s, w)
$$

where

$$
\begin{aligned}
\cot _{\bar{k}}(s, w)=\cot ^{k_{1}}\left(\frac{\pi\left(2 w-\frac{1}{2}\right)}{2}\right) \prod_{\epsilon_{1} \in\{ \pm 1\}} \cot ^{k_{2}}\left(\frac{\pi\left(s+w-\frac{1}{2}+\epsilon_{1}\left(s_{0}-\frac{1}{2}\right)\right)}{2}\right) \\
\cdot \cot ^{k_{3}}\left(\frac{\pi\left(2 s-\frac{1}{2}\right)}{2}\right) \prod_{\epsilon_{2} \in\{ \pm 1\}} \cot ^{k_{4}}\left(\frac{\pi\left(s-w+\frac{1}{2}+\epsilon_{2}\left(s_{0}-\frac{1}{2}\right)\right)}{2}\right) .
\end{aligned}
$$

Away from poles of cot we have uniform bounds $\cot \left(\frac{1}{2} \pi z\right)=i \operatorname{sign}(y)+O\left(e^{-\pi y}\right)$, so we see that $\cot _{\bar{k}}(s, w)$ is bounded in vertical strips (for the arguments away from the poles of $\cot _{\bar{k}}$ ). It follows that the functional equation (2-34) can be written simply as

$$
\begin{aligned}
& F(s, w) Z\left(s, w, \chi, \chi^{\prime}\right) \\
& \quad=\frac{G(1-s, 1-w, 0)}{G(s, w, \overline{0})} \sum_{\substack{\rho, \rho^{\prime} \bmod 8 \\
\bar{k} \in\{0,1\}^{4}}} \beta_{\bar{k}, \rho, \rho^{\prime}, \chi, \chi^{\prime}}(s, w) Z\left(1-s, 1-w, \rho, \rho^{\prime}\right),
\end{aligned}
$$

where the functions $\beta_{\bar{k}, \rho, \rho^{\prime}, \chi, \chi^{\prime}}(s, w)$ are bounded in vertical strips (away from any poles).

2E. Bounds on $Z\left(s, w, \chi, \chi^{\prime}\right)$. In this section we bound $Z\left(s, w, \chi, \chi^{\prime}\right)$ when $\mathfrak{R}(s)=\mathfrak{R}(w)=\frac{1}{2}$. Recall that in (1-6) we defined the analytic conductor to be

$$
\mathfrak{q}(t, u):=(1+|t|)(1+|t+u|)^{2}(1+|u|) .
$$

Theorem 2.17. Assume (1-4). Then

$$
Z\left(\frac{1}{2}+i t, \frac{1}{2}+i u, \chi, \chi^{\prime}\right)=O\left(\mathfrak{q}(t, u)^{\frac{1}{4}+\varepsilon}\right) .
$$


Unconditionally,

$$
Z\left(\frac{1}{2}+i t, \frac{1}{2}+i u, \chi, \chi^{\prime}\right)=O\left(\left(q(t, u)(1+|t-u|)^{2}\right)^{\frac{1}{4}+\varepsilon}\right) .
$$

We call the bound obtained in Theorem 2.17 the convexity bound. Any bound of the form $O\left(\mathfrak{q}(t, u)^{\frac{1}{4}-\delta}\right)$ is called a subconvex bound.

To prove Theorem 2.17 we first prove an approximate functional equation similar to the one in [Blomer et al. 2014, Lemma 4.2].

Lemma 2.18. Let $t, u \in \mathbb{R}$ and $\chi, \chi^{\prime} \bmod 8$. There exist smooth functions $W_{ \pm}: \mathbb{R}_{+} \rightarrow \mathbb{C}$ depending on $u, t$, and the characters satisfying

$$
y^{j} \frac{d^{j}}{d y^{j}} W_{ \pm}(y)=O(1+y)^{-A}
$$

for all $j, A \in \mathbb{N}_{0}$, uniformly in $u, t$, such that

$$
\begin{aligned}
& Z\left(\frac{1}{2}+i t, \frac{1}{2}+i u, \chi, \chi^{\prime}\right) \\
& =\sum_{\rho, \rho^{\prime} \bmod 8} \sum_{ \pm} \sum_{c=1}^{\infty} \frac{\rho^{\prime}(c) L^{* *}\left(\frac{1}{2} \pm i(t-u), \psi, c, \rho\right)}{c^{\frac{1}{2} \pm 2 i u}} W_{ \pm}\left(\frac{c}{\sqrt{\mathfrak{q}(t, u)}}\right) .
\end{aligned}
$$

Proof. Recall $1 / \cos (z)$ is holomorphic in the strip $|\Re(z)|<\pi / 2$ and satisfies $1 / \cos (z)=O_{\epsilon_{0}}\left(e^{-|z|}\right)$ for $|\Re(z)| \leq \pi / 2-\epsilon_{0}$. For $\eta(\log 2) /(\pi i)$ bounded away from $\mathbb{Z}$, the function $P_{\eta}(z)=\left(1-2^{\eta-z}\right)\left(1-2^{\eta+z}\right) /\left(1-2^{\eta}\right)^{2}$ is uniformly bounded in vertical strips, holomorphic in $\mathbb{C}$, even in $z$, with a simple zero at $\eta$, and satisfies $P_{\eta}(0)=1$. For a given multiset $B$ let

$$
H_{B}(z)=\left(\cos \left(\frac{\pi z}{3 A}\right)\right)^{-12 A} \prod_{\eta \in B} P_{\eta}(z),
$$

which is $O\left(e^{-4 \pi|z|}\right)$ for, say, $|\Re(z)| \leq\left(\frac{3}{2}-\delta\right) A$ with $\delta>0$ sufficiently small. For an appropriate choice of multiset $B=B_{t, u}$ we set $H_{t, u}(z)=H_{B_{t, u}}(z)$ so that the integrand of

$$
\frac{1}{2 \pi i} \int_{(1)} \frac{F(s+z, w+z)}{F(s, w)} Z\left(s+z, w+z, \chi, \chi^{\prime}\right) \frac{G(s+z, w+z, 0)}{G(s, w, 0)} H_{t, u}(z) \frac{d z}{z}
$$

is holomorphic in the entire $z$-plane except for a simple pole at $z=0$. (The function $H_{t, u}$ has been used to remove the poles of $Z\left(s+z, w+z, \chi, \chi^{\prime}\right)$.) Also it has rapid decay in $z$ on vertical lines due to Theorem 2.15. Moving the line of integration to $\Re(s)=-1$, we see that (2-38) equals

$$
\begin{aligned}
& Z\left(s, w, \chi, \chi^{\prime}\right) \\
& \quad+\frac{1}{2 \pi i} \int_{(-1)} \frac{F(s+z, w+z)}{F(s, w)} Z\left(s+z, w+z, \chi, \chi^{\prime}\right) \frac{G(s+z, w+z, 0)}{G(s, w, 0)} H_{t, u}(z) \frac{d z}{z} .
\end{aligned}
$$


Using the functional equation (2-36) and the change of variable $z \mapsto-z$, the last integral equals

$$
\begin{aligned}
& \sum_{\substack{\rho, \rho^{\prime} \bmod 8 \\
\bar{k} \in\{0,1\}^{4}}} \frac{1}{2 \pi i} \int_{(1)} \frac{\beta_{\bar{k}, \rho, \rho^{\prime}, \chi, \chi^{\prime}}(s-z, w-z)}{F(s, w)} Z\left(1-s+z, 1-w+z, \rho, \rho^{\prime}\right) \\
& \times \frac{G(1-s+z, 1-w+z, 0)}{G(s, w, 0)} H_{t, u}(z) \frac{d z}{z} .
\end{aligned}
$$

Thus there exist functions $\gamma_{\bar{k}, \rho, \rho^{\prime}, \chi, \chi^{\prime}, \pm}\left(x, x^{\prime}\right)$, bounded if $\Re(x)=\Re\left(x^{\prime}\right)=-\frac{1}{2}$ (note that using (2-25) we see that $F(s, w)^{-1}$ is uniformly bounded), such that $Z\left(\frac{1}{2}+i t, \frac{1}{2}+i u, \chi, \chi^{\prime}\right)$ equals

$$
\begin{aligned}
\sum_{\substack{ \pm \rho, \rho^{\prime} \bmod 8}} \frac{1}{2 \pi i} \int_{(1)} \gamma_{\bar{k}, \rho, \rho^{\prime}, \chi, \chi^{\prime}, \pm}\left(\frac{1}{2} \pm i t-z\right. & \left., \frac{1}{2} \pm i u-z\right) Z\left(\frac{1}{2} \pm i t+z, \frac{1}{2} \pm i u+z, \rho, \rho^{\prime}\right) \\
& \times \frac{G\left(\frac{1}{2} \pm i t+z, \frac{1}{2} \pm i u+z, 0\right)}{G\left(\frac{1}{2}+i t, \frac{1}{2}+i u, 0\right)} H_{t, u}(z) \frac{d z}{z} .
\end{aligned}
$$

Using the series representation (2-19) we arrive at the result with $W_{ \pm}(y)$ equal to

$$
\begin{aligned}
\sum_{\bar{k} \in\{0,1\}^{4}} \frac{1}{2 \pi i} \int_{(1)} \gamma_{\bar{k}, \rho, \rho^{\prime}, \chi, \chi^{\prime}, \pm}\left(\frac{1}{2} \pm i t-z,\right. & \left.\frac{1}{2} \pm i u-z\right)\left(y \sqrt{C^{\prime}(t, u)}\right)^{-2 z} \\
& \times \frac{G\left(\frac{1}{2} \pm i t+z, \frac{1}{2} \pm i u+z, 0\right)}{G\left(\frac{1}{2}+i t, \frac{1}{2}+i u, 0\right)} H_{t, u}(z) \frac{d z}{z} .
\end{aligned}
$$

From Stirling's formula we find that $\Gamma(s+z) / \Gamma(s)=O\left((1+|s|)^{\Re(z)} e^{\pi|z| / 2}\right)$ uniformly for $s, z$ in bounded strips away from poles. It follows that we have

$$
\frac{G\left(\frac{1}{2} \pm i t+z, \frac{1}{2} \pm i u+z, 0\right)}{G\left(\frac{1}{2}+i t, \frac{1}{2}+i u, 0\right)}=O\left(\mathfrak{q}(u, t)^{\Re(z)} e^{2 \pi|z|}\right) .
$$

By shifting the contour to $\sigma$ and differentiating under the integral sign we see that

$$
y^{j} \frac{\partial^{j} W_{ \pm}}{\partial^{j} y}=O\left(y^{-2 \sigma} \int_{(\sigma)} e^{(-4 \pi+2 \pi)|z|} \frac{(1+|z|)^{j}}{|z|} d z+\delta_{j=0, \sigma<0}\right)
$$

for $-\delta \leq \sigma<\left(\frac{3}{2}-\delta\right) A$. The last term comes from the pole at $z=0$. For $y \leq 1$ we can choose $\sigma=-\delta / 2$, and for $y>1$ we choose $\sigma=A$ and find the desired bound.

Proof of Theorem 2.17. Let $\varepsilon>0$. For $\Re(z)=\frac{1}{2}$ we have, assuming (1-4),

$$
\sum_{\substack{c \leq Y \\ c \text { odd }}}\left|L^{* *}(z, \psi, c, \rho)\right|=O\left(Y^{1+\epsilon}(1+|z|)^{a+\epsilon}\right)
$$

with $a=0$. Unconditionally, (2-39) holds with $a=\frac{1}{2}$, as is straightforward to verify from Lemma 2.6, Theorem 2.9 (2-30), and Cauchy-Schwartz. 
It follows that for an appropriate choice of $A$ in Lemma 2.18 we have

$$
\sum_{c>\mathfrak{q}(u, t)^{\frac{1}{2}+\varepsilon}} \frac{\left|L^{* *}\left(\frac{1}{2} \pm i(t-u), \psi, c, \rho\right)\right|}{c^{\frac{1}{2}}}\left|W_{ \pm}\left(\frac{c}{\sqrt{\mathfrak{q}(t, u)}}\right)\right| \leq C_{\varepsilon}\left((1+|t-u|)^{a} \mathfrak{q}(u, t)^{\epsilon}\right) .
$$

It follows also that

$$
\sum_{c \leq \mathfrak{q}(u, t)^{\frac{1}{2}+\varepsilon}} \frac{\left|L^{* *}\left(\frac{1}{2} \pm i(t-u), \psi, c, \rho\right)\right|}{c^{\frac{1}{2}}}=O\left(\mathfrak{q}(u, t)^{\frac{1}{4}+\epsilon}(1+|t-u|)^{a+\epsilon}\right) .
$$

Theorem 2.17 now follows from the approximate functional equation.

Remark 2.19. We notice that for the special configuration $w=1-s$ the conductor drops to essentially

$$
(1+|t|)(1+|u|) .
$$

This configuration will be the relevant one in Theorem 4.3 below.

Remark 2.20. One could speculate whether using another functional equation could lead to a smaller conductor. During the proof of Theorem 2.17 , or more precisely in the proof of the approximate functional equation Lemma 2.18, we have made certain choices: we have chosen a particular functional equation $(s, w) \rightarrow(1-s, 1-w)$ and a particular series representation (2-19). In principle, there is nothing that prohibits running the same type of argument with the other series representation (2-17) and/or another functional equation.

Let us consider what happens if we make other choices. If we use (2-17) and if $\Re(z)=1$ and $\Re(s)=\Re(w)=\frac{1}{2}$, then the function $Z\left(s+z, w, \chi, \chi^{\prime}\right)$ in (2-38) is evaluated in $D_{1}$, where the series representation (2-17) is convergent. Similarly, if we consider (2-19) and if $\Re(z)=1$ and $\Re(s)=\Re(w)=\frac{1}{2}$, then the function $Z\left(s+z, w+z, \chi, \chi^{\prime}\right)$ is evaluated in $D_{2}$, where the series representation (2-19) is convergent. In order for the argument in Lemma 2.18 to work we need to use a functional equation $\gamma: \mathbb{C}^{2} \rightarrow \mathbb{C}^{2}$ with the property that, when $\Re(z)=1$ and $\Re(s)=\Re(w)=\frac{1}{2}$, the numbers $\gamma(s-z, w-z) / \gamma(s-z, w)$ lie in $D_{1}$ or $D_{2}$. Only in this case is the integrand evaluated where the double Dirichlet series has a series representation (after moving the line of integration to $\Re(z)=-1$, using the functional equation and making a change of variable $z \rightarrow-z$ ).

When we are using (2-19) we assume (1-4). When we are using (2-17) we make the similar assumption for this series, namely that

$$
\sum_{\substack{n \leq X \\(n, 2)=1}}\left|t_{n} L^{*}\left(w, n, \chi^{\prime}\right)\right|=O\left(X^{1+\epsilon}(1+|w|)^{\epsilon}\right) \quad \text { for } \Re(w)=\frac{1}{2} .
$$

With these restrictions we list the possible "analytic conductors" in Table 1. 


\begin{tabular}{c|c|c}
\hline Functional equation & Series repn. & Analytic conductor \\
\hline$\beta \alpha \beta:(s, w) \rightarrow(1-s, w)$ & $(2-17)$ & $(1+|t+u|)^{2}(1+|t-u|)^{2}(1+|t|)^{2}$ \\
$\alpha \beta \alpha:(s, w) \rightarrow(1-w, 1-s)$ & $(2-19)$ & $(1+|t|)(1+|t+u|)^{2}(1+|u|)$ \\
$\alpha \beta \alpha \beta:(s, w) \rightarrow(1-s, 1-w)$ & $(2-17)$ & $(1+|t+u|)^{2}(1+|t-u|)^{2}(1+|t|)^{2}$ \\
$\alpha \beta \alpha \beta:(s, w) \rightarrow(1-s, 1-w)$ & $(2-19)$ & $(1+|t|)(1+|t+u|)^{2}(1+|u|)$ \\
\hline
\end{tabular}

Table 1. Different choices of analytic conductors.

Since, for all $t, u \in \mathbb{R}$,

$$
(1+|t|)(1+|t+u|)^{2}(1+|u|) \leq(1+|t+u|)^{2}(1+|t-u|)^{2}(1+|t|)^{2},
$$

the conductor defined in (2-37) is the smallest among these.

2F. Another double Dirichlet series. It turns out that there is another double Dirichlet series which is relevant in the applications to QUE. We now define it and then immediately show that it can be understood in terms of the series $Z\left(s, w, \chi, \chi^{\prime}\right)$ which was analyzed in the previous sections. Let

$$
\widehat{Z}\left(s, w, \chi, \chi^{\prime}\right)=\sum_{\substack{c=1 \\(c, 2)=1}} \frac{\chi^{\prime}(c) L^{*}\left(s-w+\frac{1}{2}, c, \chi\right)^{2}}{c^{2 w-\frac{1}{2}}} .
$$

In order to understand $\widehat{Z}\left(s, w, \chi, \chi^{\prime}\right)$ we exhibit an interesting nontrivial relation between the $q$-polynomials and the $Q$-polynomials in the case of the Eisenstein series when $t_{n}=\tau(n)$. Let $\widehat{Q}$ be defined as $Q$ but with the one exception that we use $\chi_{c_{0}}$ instead of $\tilde{\chi}_{c_{0}}$, i.e., with $v=v_{p}\left(c_{1}\right)$,

$$
\widehat{Q}\left(s, c_{0} c_{1}^{2}, \chi\right)=\prod_{p \mid c_{1}} \frac{t_{p^{2 v}}-t_{p^{2 v-1}} \chi_{c_{0}}(p) \chi(p)\left(p^{1-s}+p^{s}\right) / p+t_{p^{2 v-2}} \chi_{c_{0}}(p)^{2} / p}{p^{2 v\left(s-\frac{1}{2}\right)}},
$$

defined for $c_{0}, c_{1}$ odd. By (2-6) we see that

$$
\widehat{Q}(s, c, \chi)= \begin{cases}Q(s, c, \chi) & \text { if } c_{0} \equiv 1(\bmod 4), \\ Q\left(s, c, \chi \chi_{4}\right) & \text { if } c_{0} \equiv 3(\bmod 4) .\end{cases}
$$

Lemma 2.21. Let $d_{0}$ be an odd squarefree positive integer, $d_{1}$ odd, and $t_{n}=\tau(n)$. Then

$$
\sum_{d \mid d_{1}} d^{1-2 s}\left(q\left(s, d_{0} \frac{d_{1}^{2}}{d^{2}}, \chi\right)\right)^{2}=\sum_{d \mid d_{1}} \sigma_{2-4 s}(d) \widehat{Q}\left(s, d_{0} \frac{d_{1}^{2}}{d^{2}}, \chi\right) .
$$

Proof. Since the arithmetical functions involved are multiplicative, it is enough to 
verify the claim on prime powers $d_{1}=p^{n}$, i.e., we need to verify

$$
\sum_{i=0}^{n} p^{2 i\left(\frac{1}{2}-s\right)} q^{2}\left(s, d_{0} p^{2(n-i)}\right)=\sum_{i=0}^{n} \sum_{j=0}^{i} p^{4 j\left(\frac{1}{2}-s\right)} Q\left(s, d_{0} p^{2(n-i)}, \chi\right) .
$$

Using the definitions of $q(s, d, \chi)$ and $Q(s, d, \chi)$, it is a straightforward but tedious algebraic computation with sums and products of geometric sums. The details are omitted.

Using the above lemma we can now show that many properties of $\widehat{Z}\left(s, w, \chi, \chi^{\prime}\right)$ can be understood on the basis of the properties of $Z\left(s, w, \chi, \chi^{\prime}\right)$. The following lemma implies in particular that $\widehat{Z}\left(s, w, \chi, \chi^{\prime}\right)$ admits a meromorphic continuation, and that any bound we have on $Z_{\psi_{\tau}}\left(s, w, \chi, \chi^{\prime}\right)$ translates into a bound for $\widehat{Z}\left(s, w, \chi, \chi^{\prime}\right)$.

Lemma 2.22. Assume that $\psi=\psi_{\tau}$, i.e., $t_{n}=\tau(n)$. Then

$$
\begin{aligned}
\widehat{Z}\left(s, w, \chi, \chi^{\prime}\right)=\frac{1}{2 \zeta_{2}(2 s+2 w-1)}( & Z_{\psi_{\tau}}\left(s, w, \chi, \chi^{\prime}\right)+Z_{\psi_{\tau}}\left(s, w, \chi \chi_{4}, \chi^{\prime}\right) \\
& \left.+Z_{\psi_{\tau}}\left(s, w, \chi, \chi^{\prime} \chi_{4}\right)-Z_{\psi_{\tau}}\left(s, w, \chi \chi_{4}, \chi^{\prime} \chi_{4}\right)\right) .
\end{aligned}
$$

Proof. We start by noticing that $L_{2}\left(s, \chi_{c_{0}} \chi\right)^{2}=L_{2}\left(s, \psi_{\tau} \otimes \chi_{c_{0}} \chi\right)$. Now let $d_{0}$ be an odd squarefree natural number. Then

$$
\begin{aligned}
\zeta_{2}(2 s+2 w-1) \sum_{\substack{d_{1}=1 \\
d_{1} \text { odd }}}^{\infty} \frac{q^{2}\left(s, d_{0} d_{1}^{2}, \chi\right)}{d_{1}^{2 w}} & =\sum_{\substack{d, d_{1}=1 \\
d_{1}, d \text { odd }}}^{\infty} \frac{d^{1-2 s} q^{2}\left(s, d_{0} d_{1}^{2}, \chi\right)}{\left(d d_{1}\right)^{2 w}} \\
& =\sum_{\substack{d_{1}=1 \\
d_{1} \text { odd }}}^{\infty} \frac{1}{d_{1}^{2 w}} \sum_{d \mid d_{1}} d^{1-2 s} q^{2}\left(s, d_{0} d_{1}^{2} / d^{2}, \chi\right)
\end{aligned}
$$

We then use Lemma 2.21 and arrive at

$$
\begin{aligned}
\sum_{\substack{d_{1}=1 \\
d_{1} \text { odd }}}^{\infty} \frac{1}{d_{1}^{2 w}} \sum_{d \mid d_{1}} \sigma_{2-4 s}(d) \widehat{Q}\left(s, d_{0} d_{1}^{2} / d^{2}, \chi\right) & \\
& =\sum_{\substack{l=1 \\
l \text { odd }}}^{\infty} \frac{\sigma_{2-4 s}(l)}{l^{2 w}} \sum_{\substack{d_{1}=1 \\
d_{1} \text { odd }}}^{\infty} \frac{\widehat{Q}\left(s, d_{0} d_{1}^{2}, \chi\right)}{d_{1}^{2 w}} \\
& =\zeta_{2}(4 s+2 w-2) \zeta_{2}(2 w) \sum_{\substack{d_{1}=1 \\
d_{1} \text { odd }}}^{\infty} \frac{\widehat{Q}\left(s, d_{0} d_{1}^{2}, \chi\right)}{d_{1}^{2 w}} .
\end{aligned}
$$

Multiply the first and last expression by $\chi^{\prime}\left(d_{0}\right) L_{2}\left(s, \chi_{d_{0}} \chi\right)^{2} / d_{0}^{w}$, then summing 
over all odd squarefree natural numbers $d_{0}$ we get

$$
\begin{aligned}
& \zeta_{2}(2 s+2 w-1) \sum_{\substack{d=1 \\
(d, 2)=1}}^{\infty} \frac{\chi^{\prime}(d) q^{2}(s, d, \chi) L_{2}\left(s, \chi_{d_{0}} \chi\right)^{2}}{d^{w}} \\
&=\zeta_{2}(4 s+2 w-2) \zeta_{2}(2 w) \sum_{\substack{d=1 \\
(d, 2)=1}}^{\infty} \frac{\chi^{\prime}(d) \widehat{Q}(s, d, \chi) L_{2}\left(s, \psi_{\tau} \otimes \chi_{d_{0}} \chi\right)}{d^{w}} .
\end{aligned}
$$

By (2-6) we see that

$$
\begin{aligned}
\widehat{Q}(s, d, \chi) L_{2}\left(s, \psi_{\tau} \otimes \chi_{d_{0}} \chi\right) & \begin{array}{ll}
Q_{\psi_{\tau}}(s, d, \chi) L_{2}\left(s, \psi_{\tau} \otimes \chi_{d_{0}} \chi\right) & \text { if } d \equiv 1(\bmod 4), \\
Q_{\psi_{\tau}}\left(s, d, \chi \chi_{4}\right) L_{2}\left(s, \psi_{\tau} \otimes \chi_{d_{0}} \chi \chi_{4}\right) & \text { if } d \equiv 3(\bmod 4) .
\end{array}
\end{aligned}
$$

Substituting $\left(s-w+\frac{1}{2}, 2 w-\frac{1}{2}\right)$ for $(s, w)$ and comparing with (2-18), we obtain the desired result.

\section{Eisenstein series}

We briefly recall a few facts about Eisenstein series with weights. For $\gamma \in \mathrm{SL}_{2}(\mathbb{R})$ and $z \in \mathbb{H}$ we define $j(\gamma, z)=c z+d$ and $j_{\gamma}(z)=(c z+d) /|c z+d|$. We let arg denote the principal argument and define $j_{\gamma}(z)^{k}=e^{i k \arg (c z+d)}$. Since $j\left(\gamma_{1} \gamma_{2}, z\right)=j\left(\gamma_{1}, \gamma_{2} z\right) j\left(\gamma_{2}, z\right)$,

$$
\widetilde{\omega}\left(\gamma_{1}, \gamma_{2}\right)=\frac{1}{2 \pi}\left(\arg j\left(\gamma_{1}, \gamma_{2} z\right)+\arg j\left(\gamma_{2}, z\right)-\arg j\left(\gamma_{1} \gamma_{2}, z\right)\right)
$$

is an integer independent of $z$. The factor system of weight $k \in \mathbb{R}$ is then defined as

$$
\omega\left(\gamma_{1}, \gamma_{2}\right)=e\left(k \tilde{\omega}\left(\gamma_{1}, \gamma_{2}\right)\right) .
$$

Then we have $\omega\left(\gamma_{1}, \gamma_{2}\right) j_{\gamma_{1} \gamma_{2}}(z)^{k}=j_{\gamma_{1}}\left(\gamma_{2} z\right)^{k} j_{\gamma_{2}}(z)^{k}$. We refer to [Iwaniec 1997, Chapters 2.6, 3] for the basic properties of multiplier systems as well as for further explanations of the generalities of Fourier expansions.

Let $v$ be a weight $k$ multiplier system, and let $\Gamma$ be a cofinite subgroup of $\mathrm{SL}_{2}(\mathbb{R})$. For an open cusp $\mathfrak{a}$, i.e., $v(\mathfrak{a})=1$, we define the weight $k$ Eisenstein series for $\Gamma$ by

$$
E_{\mathfrak{a}}(z, s, k):=\sum_{\gamma \in \Gamma_{\mathfrak{a}} \backslash \Gamma} \overline{v(\gamma) \omega\left(\sigma_{\mathfrak{a}}^{-1}, \gamma\right)} j_{\sigma_{\mathfrak{a}}^{-1} \gamma}(z)^{-k} \mathfrak{\Im}\left(\sigma_{\mathfrak{a}}^{-1} \gamma z\right)^{s} \quad \text { for } \Re(s)>1,
$$

where $\sigma_{\mathfrak{a}}$ is a scaling matrix of the cusp a, i.e., $\sigma_{\mathfrak{a}}^{-1} \Gamma_{\mathfrak{a}} \sigma_{\mathfrak{a}}=\Gamma_{\infty}$, with $\Gamma_{\infty}$ being generated by $\gamma_{\infty}=\left(\begin{array}{ll}1 & 1 \\ 0 & 1\end{array}\right)$ and $-\gamma_{\infty}$ if $-I \in \Gamma$. The function satisfies, for $\gamma \in \Gamma$, $E_{\mathfrak{a}}(\gamma z, s, k)=v(\gamma) j_{\gamma}^{k}(z) E_{\mathfrak{a}}(z, s, k)$, it is an eigenfunction of the weight $k$ Laplacian with eigenvalue $s(1-s)$, and admits a meromorphic continuation to $s \in \mathbb{C}$. We now 
briefly recall how to find the Fourier coefficients of $E_{\mathfrak{a}}(z, s, k)$ at an open cusp $\mathfrak{b}$. We have

$$
j_{\sigma_{\mathfrak{b}}}(z)^{-k} E_{\mathfrak{a}}\left(\sigma_{\mathfrak{b}} z, s, k\right)=\sum_{\gamma \in \Gamma_{\infty} \backslash \sigma_{\mathfrak{a}}^{-1} \Gamma \sigma_{\mathfrak{b}}} \overline{v_{\mathfrak{a} \mathfrak{b}}(\gamma)} j_{\gamma}(z)^{-k} \Im(\gamma z)^{s},
$$

where $v_{\mathfrak{a} \mathfrak{b}}(\gamma)=v\left(\sigma_{\mathfrak{a}} \gamma{\sigma_{\mathfrak{b}}}^{-1}\right) \omega\left(\sigma_{\mathfrak{a}}^{-1}, \sigma_{\mathfrak{a}} \gamma \sigma_{\mathfrak{b}}^{-1}\right) \omega\left(\gamma \sigma_{\mathfrak{b}}^{-1}, \sigma_{\mathfrak{b}}\right)$. For the rest of the paper we can assume that $-I \in \Gamma$. Summing over a set of representatives of $\Gamma_{\infty} \backslash \sigma_{\mathfrak{a}}^{-1} \Gamma \sigma_{\mathfrak{b}} / \Gamma_{\infty}$, which we can assume have $c_{\gamma}>0$ for $\gamma \notin \Gamma_{\infty}$, we see that

$$
j_{\sigma_{\mathfrak{b}}}(z)^{-k} E_{\mathfrak{a}}\left(\sigma_{\mathfrak{b}} z, s, k\right)=\delta_{\mathfrak{a}=\mathfrak{b}} y^{s}+\sum_{I \neq \gamma \in \Gamma_{\infty} \backslash \sigma_{\mathfrak{a}}^{-1} \Gamma \sigma_{\mathfrak{b}} / \Gamma_{\infty}} \overline{v_{\mathfrak{a} \mathfrak{b}}(\gamma)} \sum_{l \in \mathbb{Z}} j_{\gamma \gamma_{\infty}^{l}}(z)^{-k} \Im\left(\gamma \gamma_{\infty}^{l} z\right)^{s} .
$$

Therefore, by a familiar computation, we have

$$
\begin{aligned}
& \int_{0}^{1}\left(j_{\sigma_{\mathfrak{b}}}(z)^{-k} E_{\mathfrak{a}}\left(\sigma_{\mathfrak{b}} z, s, k\right)-\delta_{\mathfrak{a}=\mathfrak{b}} y^{s}\right) e(-n x) d x \\
&=\sum_{I \neq \gamma \in \Gamma_{\infty} \backslash \sigma_{\mathfrak{a}}^{-1} \Gamma \sigma_{\mathfrak{b}} / \Gamma_{\infty}} \frac{\overline{v_{\mathfrak{a} \mathfrak{b}}(\gamma)}}{c^{2 s}} e\left(n \frac{d}{c}\right) y^{s} \int_{-\infty}^{\infty}\left(\frac{z}{|z|}\right)^{-k} \frac{1}{|z|^{2 s}} e(-n x) d x .
\end{aligned}
$$

Substituting $t=x / y$ in the last integral we see that

$$
\begin{aligned}
y^{s} \int_{-\infty}^{\infty}\left(\frac{z}{|z|}\right)^{-k} \frac{e(-n x)}{|z|^{2 s}} d x & =y^{1-s} \int_{-\infty}^{\infty}\left(\frac{t+i}{|t+i|}\right)^{-k} \frac{e(-n t y)}{|t+i|^{2 s}} d t \\
& =e^{-i k \pi / 2} y^{1-s} \int_{-\infty}^{\infty}\left(\frac{1-i t}{|1-i t|}\right)^{-k} \frac{e(-n t y)}{|1+i t|^{2 s}} d t \\
& = \begin{cases}\pi^{s} e^{-i k \pi / 2} \frac{|n|^{s-1}}{\Gamma\left(s+\frac{k n}{2|n|}\right)} W_{\frac{k n}{2 \mid n}, s-\frac{1}{2}}(4 \pi|n| y) & \text { if } n \neq 0, \\
\pi 4^{1-s} e^{-i k \pi / 2} \frac{\Gamma(2 s-1) y^{1-s}}{\Gamma\left(s+\frac{k}{2}\right) \Gamma\left(s-\frac{k}{2}\right)} & \text { if } n=0,\end{cases}
\end{aligned}
$$

where $W_{\mu, v}(y)$ is the Whittaker function and where we have used [Gradshteyn and Ryzhik 2007, 3.384 (9), p. 349] for $n \neq 0$ and [Shimura 1975, p. 84-85] for $n=0$.

3A. Eisenstein series of level 4. We now specialize to $\Gamma=\Gamma_{0}(4)$. In this case the Fourier coefficients of half-integral weight Eisenstein series were originally studied by Shimura [1975]. We consider the weight $\frac{1}{2}$ multiplier system $v$ related to the theta series

$$
\theta(z):=y^{\frac{1}{4}} \sum_{m \in \mathbb{Z}} e\left(m^{2} z\right)
$$


i.e., $\theta(\gamma z)=v(\gamma) j_{\gamma}(z)^{\frac{1}{2}} \theta(z)$ for $\gamma \in \Gamma$. It is well known that

$$
v(\gamma)=\left(\frac{c}{d}\right) \varepsilon_{d}^{-1} \text { for }\left(\begin{array}{ll}
a & b \\
c & d
\end{array}\right)=\gamma \in \Gamma_{0}(4)
$$

Here the Jacobi-Legendre symbol is extended as in [Shimura 1973, p. 442]. The group $\Gamma_{0}(4)$ has 3 cusps, $\mathfrak{a}_{1}=\infty, \mathfrak{a}_{2}=0, \mathfrak{a}_{3}=\frac{1}{2}$, with corresponding stabilizers $\Gamma_{\mathfrak{a}_{i}}$ generated by $\pm \gamma_{\mathfrak{a}_{i}}$ where

$$
\gamma_{\mathfrak{a}_{1}}=\left(\begin{array}{ll}
1 & 1 \\
0 & 1
\end{array}\right), \quad \gamma_{\mathfrak{a}_{2}}=\left(\begin{array}{cc}
1 & 0 \\
-4 & 1
\end{array}\right), \quad \gamma_{\mathfrak{a}_{3}}=\left(\begin{array}{cc}
-1 & 1 \\
-4 & 3
\end{array}\right)
$$

and we define scaling matrices

$$
\sigma_{\mathfrak{a}_{1}}=\left(\begin{array}{ll}
1 & 0 \\
0 & 1
\end{array}\right), \quad \sigma_{\mathfrak{a}_{2}}=\left(\begin{array}{cc}
0 & -\frac{1}{2} \\
2 & 0
\end{array}\right), \quad \sigma_{\mathfrak{a}_{3}}=\left(\begin{array}{cc}
1 & -\frac{1}{2} \\
2 & 0
\end{array}\right)
$$

Only the cusps $\infty$ and 0 are open with respect to $v$, as

$$
v\left(\gamma_{\mathfrak{a}_{1}}\right)=v\left(\gamma_{\mathfrak{a}_{2}}\right)=1, \quad v\left(\gamma_{\mathfrak{a}_{3}}\right)=\left(\frac{-4}{3}\right) \epsilon_{3}^{-1}=i
$$

We now compute the Fourier expansion for the weight $\frac{1}{2}$ Eisenstein series. We focus on the cusp at infinity but the analysis for the other cusps is similar, although slightly more technical. The main extra complication at the other cusps comes from the factor system. This can be dealt with as follows: For $k=\frac{1}{2}$ we can use $z=\gamma_{2}^{-1} i$ in the definition of the factor system to see that

$$
\omega\left(\gamma_{1}, \gamma_{2}\right)= \begin{cases}1 & \text { if }-\pi<\arg \left(c_{\gamma_{1}} i+d_{\gamma_{1}}\right)+\arg \left(c_{\gamma_{2}} i+a_{\gamma_{2}}\right) \leq \pi \\ -1 & \text { otherwise }\end{cases}
$$

Using the properties of a multiplier system one finds (see [Iwaniec 1997, (3.5)]) that

$$
v_{\mathfrak{a} \mathfrak{b}}(\gamma)=v\left(\sigma_{\mathfrak{a}} \gamma \sigma_{\mathfrak{b}}^{-1}\right) \frac{\omega\left(\sigma_{\mathfrak{a}} \gamma \sigma_{\mathfrak{b}}^{-1}, \sigma_{\mathfrak{b}}\right)}{\omega\left(\sigma_{\mathfrak{a}}, \gamma\right)}
$$

This is explicit enough that one can do the computations also for the other cusps.

We now focus on $\left(\mathfrak{a}_{1}, \mathfrak{a}_{1}\right)=(\infty, \infty)$, and omit the corresponding subscripts. Using that all the nonidentity elements of $\Gamma_{\infty} \backslash \Gamma / \Gamma_{\infty}$ are parametrized by $\left(\begin{array}{cc}* & * \\ 4 c & d\end{array}\right)$ with $c>0, d \bmod 4 c,(d, 4 c)=1$, we find that

$$
E\left(z, s, \frac{1}{2}\right)=y^{s}+\phi\left(s, \frac{1}{2}\right) y^{1-s}+\sum_{n \neq 0} \phi_{n}\left(s, \frac{1}{2}\right) W_{n /(4|n|), s-\frac{1}{2}}(4 \pi|n| y) e(n x)
$$


with

and

$$
\begin{aligned}
\phi_{n}\left(s, \frac{1}{2}\right) & =\frac{\pi^{s} e^{-i \pi / 4}|n|^{s-1}}{\Gamma\left(s+\frac{n}{4|n|}\right)} \sum_{c=1}^{\infty} \frac{1}{(4 c)^{2 s}} \sum_{\substack{d \bmod 4 c \\
(d, 4 c)=1}} v \overline{\left(\begin{array}{cc}
* & * \\
4 c & d
\end{array}\right)} e(n d / 4 c) \\
& =\frac{\pi^{s} e^{-i \pi / 4}|n|^{s-1}}{\Gamma\left(s+\frac{n}{4|n|}\right)} \sum_{c=1}^{\infty} \frac{1}{(4 c)^{2 s}} \sum_{d \bmod 4 c} \varepsilon_{d}\left(\frac{4 c}{d}\right) e(n d / 4 c),
\end{aligned}
$$

$$
\phi\left(s, \frac{1}{2}\right)=\frac{\pi 4^{1-s} e^{-i \pi / 4} \Gamma(2 s-1)}{\Gamma\left(s+\frac{1}{4}\right) \Gamma\left(s-\frac{1}{4}\right)} \sum_{c=1}^{\infty} \frac{1}{(4 c)^{2 s}} \sum_{d \bmod 4 c} \varepsilon_{d}\left(\frac{4 c}{d}\right) .
$$

If we write $4 c=2^{k} c^{\prime}$ with $c^{\prime}$ odd then Sturm proved [1980, Lemma 1] —using quadratic reciprocity and the Chinese remainder theorem - that

$$
\sum_{d \bmod 4 c} \varepsilon_{d}\left(\frac{4 c}{d}\right) e(n d / 4 c)=H_{n}\left(c^{\prime}\right) \sum_{r \bmod 2^{k}}\left(\frac{2^{k}}{r}\right) \varepsilon_{r} e\left(n r / 2^{k}\right) .
$$

It follows that, for $n \neq 0$,

$$
\phi_{n}\left(s, \frac{1}{2}\right)=\frac{\pi^{s} e^{-i \pi / 4}|n|^{s-1}}{\Gamma\left(s+\frac{n}{4|n|}\right)} \sum_{\substack{c^{\prime}=1 \\\left(c^{\prime}, 2\right)=1}}^{\infty} \frac{H_{n}\left(c^{\prime}\right)}{c^{\prime 2 s}} \sum_{k=2}^{\infty} \frac{\sum_{r \bmod 2^{k}}\left(\frac{2^{k}}{r}\right) \varepsilon_{r} e\left(n r / 2^{k}\right)}{2^{2 k s}},
$$

which by Lemma 2.2 equals

$$
\frac{\pi^{s} e^{-i \pi / 4}|n|^{s-1}}{\Gamma\left(s+\frac{n}{4|n|}\right)} \frac{L^{*}\left(2 s-\frac{1}{2}, n, 1\right)}{\zeta_{2}(4 s-1)} r_{2}(s, n),
$$

where we have written

$$
r_{2}(s, n):=\sum_{k=2}^{\infty} \frac{\sum_{r \bmod 2^{k}}\left(\frac{2^{k}}{r}\right) \varepsilon_{r} e\left(n r / 2^{k}\right)}{2^{k 2 s}} .
$$

The function $r_{2}(s, n)$ can also be computed. One uses that $\varepsilon_{d}$ can be expressed as a sum of characters mod 4 as

$$
\varepsilon_{d}=\frac{1}{2}(1+i) \chi_{4}^{0}(d)+\frac{1}{2}(1-i) \chi_{4}(d) .
$$

Inserting this in (3-4) the numerator becomes

$$
\frac{1}{2}(1+i) G_{n}\left(\chi_{8}^{k} \chi_{2^{k}}^{0}\right)+\frac{1}{2}(1-i) G_{n}\left(\chi_{8}^{k} \chi_{4} \chi_{2^{k}}^{0}\right),
$$

where $\chi_{8}$ is the primitive character mod 8 given by $\chi_{8}(n)=(-1)^{\frac{1}{8}(n-1)(n+1)}$ for $(n, 2)=1$, and the $G_{n}$ denote the usual Gauss sums. Using [Shimura 1975, Lemma 3] as well as explicit computations of $G_{1}\left(\chi_{1}\right), G_{1}\left(\chi_{8}\right), G_{1}\left(\chi_{4}\right), G_{1}\left(\chi_{4} \chi_{8}\right)$, 
these can all be computed and using the result one can compute $r_{2}(s, n)$. We omit the details but state the result. Assume first $n \not \equiv 0(\bmod 4)$. Then

$$
r_{2}(s, n)=\frac{1}{4}(1+i) \begin{cases}-\frac{1}{2^{2(2 s-1)}} & n \neq 1(\bmod 4), \\ \frac{1}{2^{2(2 s-1)}}+\frac{\chi_{8}(n) \sqrt{2}}{2^{3(2 s-1)}} & n \equiv 1(\bmod 4) .\end{cases}
$$

More generally we find that, if $n=4^{r} n_{0}$ with $n_{0} \not \equiv 0(\bmod 4)$, then

$$
r_{2}(s, n)=\frac{1}{4}(1+i) u_{r}\left(2^{-(2 s-1)}\right)+4^{-r(2 s-1)} r_{2}\left(s, n_{0}\right),
$$

where

$$
u_{r}(x)=\frac{\left(x^{2}\right)^{r+1}-x^{2}}{x^{2}-1}
$$

We remark that $r_{2}(s, n)$ is entire.

3A1. Scattering term. We now compute the scattering term $\phi\left(s, \frac{1}{2}\right)$, which by (3-2) equals

$$
\frac{\pi 4^{1-s} e^{-i \pi / 4} \Gamma(2 s-1)}{\Gamma\left(s+\frac{1}{4}\right) \Gamma\left(s-\frac{1}{4}\right)} \sum_{\substack{c^{\prime}=1 \\\left(c^{\prime}, 2\right)=1}}^{\infty} \frac{H_{0}\left(c^{\prime}\right)}{c^{\prime 2 s}} \sum_{k=2}^{\infty} \sum_{r \bmod 2^{k}}\left(\frac{2^{k}}{r}\right) \frac{\varepsilon_{r}}{2^{2 k s}} .
$$

The sum $\sum_{\substack{c^{\prime}=1 \\\left(c^{\prime}=2\right)=1}}^{\infty} \frac{H_{0}\left(c^{\prime}\right)}{c^{\prime 2 s}}$ factors, and for an odd prime $p$ we observe that

$$
H_{0}\left(p^{\beta}\right)= \begin{cases}\varphi\left(p^{\beta}\right) & \text { if } \beta \equiv 0(\bmod 2) \\ 0 & \text { otherwise }\end{cases}
$$

Here $\varphi$ is Euler's $\varphi$-function. Therefore

$$
\sum_{\beta=0}^{\infty} \frac{H_{0}\left(p^{\beta}\right)}{p^{\beta 2 s}}=\sum_{\beta=0}^{\infty} \frac{\varphi\left(p^{2 \beta}\right)}{p^{2 \beta 2 s}}=\frac{\zeta^{(p)}(4 s-2)}{\zeta^{(p)}(4 s-1)} .
$$

For the prime 2 we note that for $k \geq 2$ we have

$$
G_{0}\left(\chi_{4} \chi_{2^{k}}^{0}\right)=G_{0}\left(\chi_{8} \chi_{2^{k+1}}^{0}\right)=G_{0}\left(\chi_{4} \chi_{8} \chi_{2^{k+1}}^{0}\right)=0
$$

Using this, we find

$$
\begin{aligned}
\sum_{k=2}^{\infty} & \sum_{r}\left(\frac{2^{k}}{r}\right) \frac{\varepsilon_{r}}{2^{k 2 s}} \\
= & \sum_{\substack{k=2 \\
k \text { even }}}^{\infty} \frac{\frac{1+i}{2} G_{0}\left(\chi_{4}^{0} \chi_{2^{k}}^{0}\right)+\frac{1-i}{2} G_{0}\left(\chi_{4} \chi_{2^{k}}^{0}\right)}{2^{k 2 s}}+\sum_{\substack{k=2 \\
k \text { odd }}}^{\infty} \frac{\frac{1+i}{2} G_{0}\left(\chi_{8} \chi_{2^{k}}^{0}\right)+\frac{1-i}{2} G_{0}\left(\chi_{8} \chi_{4} \chi_{2^{k}}^{0}\right)}{2^{k s}} \\
= & \sum_{\substack{k=2 \\
k \text { even }}}^{\infty} \frac{\frac{1+i}{2} \varphi\left(2^{k}\right)}{2^{k 2 s}}=(1+i) \frac{2^{-4 s}}{1-2^{-(4 s-2)}} .
\end{aligned}
$$


It follows that

$$
\phi\left(s, \frac{1}{2}\right)=\pi 4^{1-s} e^{-i \pi / 4} \frac{\Gamma(2 s-1)}{\Gamma\left(s+\frac{1}{4}\right) \Gamma\left(s-\frac{1}{4}\right)} \frac{(1+i)}{2^{4 s}} \frac{\zeta(4 s-2)}{\zeta_{2}(4 s-1)} .
$$

Using that $\Gamma\left(s+\frac{1}{4}\right) \Gamma\left(s-\frac{1}{4}\right)=\sqrt{\pi} 2^{3 / 2-2 s} \Gamma\left(2 s-\frac{1}{2}\right)$, this simplifies to

$$
\frac{1}{2^{4 s-1}-1} \frac{\xi(4 s-2)}{\xi(4 s-1)},
$$

where $\xi(s)=\pi^{-s / 2} \Gamma(s / 2) \zeta(s)$ (compare [Iwaniec 1997, p. 247-248]). The other entries in the scattering matrix $\Phi\left(s, \frac{1}{2}\right)$ can be computed in a similar way and we find

$$
\Phi\left(s, \frac{1}{2}\right)=\left(\begin{array}{cc}
\frac{2^{-(4 s-1)}}{1-2^{-(4 s-2)}} & \frac{1-i}{2^{2 s}} \\
\frac{1+i}{2^{2 s}} & \frac{2^{-(4 s-1)}}{1-2^{-(4 s-2)}}
\end{array}\right) \frac{1-2^{-(4 s-2)}}{1-2^{-(4 s-1)}} \frac{\xi(4 s-2)}{\xi(4 s-1)} .
$$

As a consistency check we note that a direct computation and the functional equation for $\xi$ show that the scattering matrix verifies $\Phi\left(s, \frac{1}{2}\right) \Phi\left(1-s, \frac{1}{2}\right)=I$, as predicted by the general theory.

3B. Eisenstein series of level $2^{n}$. We now consider the group $\Gamma_{0}(N)$, where $N=2^{n}$ with $n \geq 2$. Let $\chi$ be a Dirichlet character modulo $N$, and consider the weight $\frac{1}{2}$ multiplier system

$$
v(\gamma)=\chi(d)\left(\frac{c}{d}\right) \varepsilon_{d}^{-1} \text { for }\left(\begin{array}{ll}
a & b \\
c & d
\end{array}\right)=\gamma \in \Gamma_{0}(N) .
$$

We consider the corresponding Eisenstein series of weight $\frac{1}{2}$ at the cusp at 0 , denoted by

$$
E_{0, \chi}\left(z, s, \frac{1}{2}\right) \text {. }
$$

Similarly one denotes $E_{\infty, \chi}\left(z, s, \frac{1}{2}\right)$ the corresponding Eisenstein series at the cusp $\infty$. The Fourier coefficients at infinity of the Eisenstein series at zero has a simpler 2-factor than the Eisenstein series at infinity. The stabilizer at 0 is generated by $\pm \gamma_{0}$ and has corresponding scaling matrix $\sigma_{0}$, where

$$
\gamma_{0}=\left(\begin{array}{cc}
1 & 0 \\
-2^{n} & 1
\end{array}\right), \quad \sigma_{0}=\left(\begin{array}{cc}
0 & -1 / \sqrt{2^{n}} \\
\sqrt{2^{n}} & 0
\end{array}\right) .
$$

From the general considerations in the beginning of Section 3 we find that the nonzero Fourier coefficients at infinity equal

$$
\sum_{I \neq \gamma \in \Gamma_{\infty} \backslash \sigma_{0}^{-1} \Gamma_{0}(N) / \Gamma_{\infty}} \frac{\overline{\nu_{0 \infty}(\gamma)}}{c^{2 s}} e\left(n \frac{d}{c}\right) \pi^{s} e^{-i \pi / 4} \frac{|n|^{s-1}}{\Gamma\left(s+\frac{n}{4|n|}\right)} W_{\frac{n}{4|n|}, s-\frac{1}{2}}(4 \pi|n| y) .
$$


After some computations one finds

$$
\begin{aligned}
& \sum_{I \neq \gamma \in \Gamma_{\infty} \backslash \sigma_{0}^{-1} \Gamma_{0}(N) / \Gamma_{\infty}} \frac{\overline{v_{0 \infty}(\gamma)}}{c^{2 s}} e\left(n \frac{d}{c}\right)=\frac{i \chi(-1)}{N^{s}} \sum_{\substack{a=1 \\
(a, 2)=1}}^{\infty} \frac{\chi(a) H_{n}(a)}{a^{2 s}} \\
& =\frac{i \chi(-1)}{N^{s}} \frac{L^{*}\left(2 s-\frac{1}{2}, n, \chi\right)}{\zeta_{2}(4 s-1)},
\end{aligned}
$$

where in the last equality we have used Lemma 2.2. Using this it is straightforward to see how $Z\left(s, w, \chi, \chi^{\prime}\right)$ relates directly to a Rankin-Selberg integral in the case where $\left\{t_{n}\right\}$ comes from a cusp form. Let $\psi$ be a cuspidal Hecke newform of weight zero, and trivial multiplier for $\Gamma_{0}\left(2^{k}\right)$ with eigenvalue $s_{0}\left(1-s_{0}\right)$ and Fourier expansion

$$
\psi(z)=\sum_{n \neq 0} b_{n} W_{0, s_{0}-\frac{1}{2}}(4 \pi|n| y) e(n x) .
$$

Let $\chi$ be a Dirichlet character mod 8. Consider the twisted Maaß form

$$
\psi \otimes \chi(z)=\sum_{n \neq 0} \chi(n) b_{n} W_{0, s_{0}-\frac{1}{2}}(4 \pi|n| y) e(n x),
$$

which is a weight zero cusp form for some $\Gamma_{0}(M)$ and character $\chi_{0}^{M}$ for some $M \mid \operatorname{lcm}\left(64,2^{k}\right)$ and $8 \mid M$. Let $\chi^{\prime}$ be another Dirichlet character mod 8. Consider now the Rankin-Selberg integral

$$
I\left(\psi, \chi, \chi^{\prime}, s, w\right)=\int_{\Gamma_{0}(M) \backslash \mathbb{E}} \psi \otimes \chi(z) E_{0, \chi_{0}^{M} \chi^{\prime}}\left(z, w, \frac{1}{2}\right) \overline{E_{\infty, \chi_{0}^{M} \chi^{\prime}}\left(z, \bar{s}, \frac{1}{2}\right)} d \mu(z) .
$$

This is the integral studied by Friedberg and Hoffstein [1995, (1.2) p. 388].

Unfolding, using $b_{n}=b_{n /|n|}|n|^{-\frac{1}{2}} t_{|n|},(3-11)$, and $L^{*}(s,-n, \chi)=L^{*}\left(s, n, \chi_{4} \chi\right)$ we arrive at

$$
\begin{aligned}
& I\left(\psi, \chi, \chi^{\prime}, s, w\right) \\
& =\frac{\pi^{w} e^{-i \pi / 4} i \chi^{\prime}(-1)}{(2 \pi)^{s-1} M^{w} \zeta_{2}(4 w-1)} \sum_{\substack{n \neq 0 \\
(n, 2)=1}} \frac{\chi(n) b_{n /|n|} t_{|n|} L^{*}\left(2 w-\frac{1}{2}, n, \chi^{\prime}\right)}{|n|^{s-w+\frac{1}{2}}} G_{\frac{n}{|n|}}(w) \\
& =\frac{\pi^{w} e^{-i \pi / 4} i \chi^{\prime}(-1)\left[Z\left(s, w, \chi, \chi^{\prime}\right) G_{+}(w)\right.}{(2 \pi)^{s-1} M^{w} \zeta_{2}(4 w-1) \zeta_{2}(4 s-1)},
\end{aligned}
$$

where

$$
G_{ \pm}(w)=\frac{1}{\Gamma\left(w \pm \frac{1}{4}\right)} \int_{0}^{\infty} W_{ \pm \frac{1}{4}, w-\frac{1}{2}}(2 y) W_{0, s_{0}-\frac{1}{2}}(2 y) y^{w-1} \frac{d y}{y} .
$$

Lemma 3.1. $I\left(\psi, \chi, \chi^{\prime}, \frac{1}{2}+i t, \frac{1}{2}+i u\right)=O(\log ((2+|t|)(2+|u|)))$. 
Proof. This follows from the Maaß-Selberg relation, and known properties of the relevant scattering matrix.

It is tempting to speculate whether the above bound on $I\left(\psi, \chi, \chi^{\prime}, \frac{1}{2}+i t, \frac{1}{2}+i u\right)$ can be used to bound $Z\left(s, w, \chi, \chi^{\prime}\right)$ through (3-13). What we can prove is the following:

Denote the expression in the square brackets of (3-13) by $\tilde{I}\left(\psi, \chi, \chi^{\prime}, s, w\right)$. We then find that

$$
\begin{aligned}
& \tilde{I}\left(\psi, \chi, \chi^{\prime}, s, w\right) \pm \tilde{I}\left(\psi, \chi, \chi_{4} \chi^{\prime}, s, w\right) \\
& \quad=\left(Z\left(s, w, \chi, \chi^{\prime}\right) \pm Z\left(s, w, \chi, \chi_{4} \chi^{\prime}\right)\right)\left(G_{+}(w) \pm \chi(-1) b_{-1} G_{-}(w)\right) .
\end{aligned}
$$

Lemma 3.2. Assume that $\psi$ is a cusp form. Then, for $s=1-w=\frac{1}{2}+i t$,

$$
Z\left(s, w, \chi, \chi^{\prime}\right)+\chi(-1) b_{-1} Z\left(s, w, \chi, \chi_{4} \chi^{\prime}\right)=O\left((1+|t|)^{\frac{1}{2}+\epsilon}\right) .
$$

Proof. From (3-14) we see that

$$
\begin{array}{r}
Z\left(s, w, \chi, \chi^{\prime}\right)+\chi(-1) b_{-1} Z\left(s, w, \chi, \chi_{4} \chi^{\prime}\right)\left(G_{+}(w)+G_{-}(w)\right) \\
=\tilde{I}\left(\psi, \chi, \chi^{\prime}, s, w\right)+\chi(-1) b_{-1} \tilde{I}\left(\psi, \chi, \chi_{4} \chi^{\prime}, s, w\right) .
\end{array}
$$

The claim now follows from Lemmas 3.1 and A.1, combined with Remark A.2.

Remark 3.3. We notice that with the restriction above on $s, w$ the conductor $\mathfrak{q}(t,-t)$ is of order $(1+|t|)^{2}$. So the right-hand side in (3-15) is of order $\mathfrak{q}(t,-t)^{\frac{1}{4}+\epsilon}$, i.e., for the linear combination $Z\left(s, w, \chi, \chi^{\prime}\right)+\chi(-1) b_{-1} Z\left(s, w, \chi, \chi_{4} \chi^{\prime}\right)$ we have proved the convexity estimate unconditionally. Surprisingly this "soft" method of using the Maaß-Selberg relations gives much stronger bounds than the harder method using Heath-Brown's equation (2-29) and approximate functional equations. Unfortunately we do not know how to prove this unconditionally for $Z\left(s, w, \chi, \chi^{\prime}\right)$ and $Z\left(s, w, \chi, \chi_{4} \chi^{\prime}\right)$ separately. The main reason for this is that $G_{+}(w)-G_{-}(w)$ decays much faster than $G_{+}(w)+G_{-}(w)$, so using a similar argument on

$$
Z\left(s, w, \chi, \chi^{\prime}\right)-\chi(-1) b_{-1} Z\left(s, w, \chi, \chi_{4} \chi^{\prime}\right)
$$

gives very poor bounds.

If we use (2-19) (i.e., interchange sums) we find, like [Friedberg and Hoffstein 1995, (1.2) p. 389], that $\tilde{I}\left(\psi, \chi, \chi^{\prime}, s, w\right)$ equals

$$
\sum_{\substack{c=1 \\(c, 2)=1}} \frac{\chi^{\prime}(c) L^{* *}\left(s-w+\frac{1}{2}, \psi, c, \chi\right)}{c^{2 w-\frac{1}{2}}}\left(G_{+}(w)+\chi(-1) b_{-1} \chi_{4}(c) G_{-}(w)\right) .
$$

By taking linear combinations over different $\chi^{\prime}$ we can restrict to $c$ in a specific residue class, as in the work of [Friedberg and Hoffstein 1995]. 


\section{Limits of weight $\frac{1}{2}$ Eisenstein series}

We consider separately Maaß cusp forms and incomplete Eisenstein series, i.e., we analyze

$$
\int_{\Gamma \backslash \mathbb{\uplus}} \psi(z)\left|E\left(z, \frac{1}{2}+i t, \frac{1}{2}\right)\right|^{2} d \mu(z),
$$

where $\psi$ is either a Maaß cusp form or an incomplete Eisenstein series. Then a standard approximation argument — see [Luo and Sarnak 1995, p. 217] — implies the result (1-10).

4A. The cuspidal contribution. Let $\psi$ be a cuspidal element of a weight zero Hecke basis for $\Gamma_{0}(4)$ with eigenvalue $s_{0}\left(1-s_{0}\right)$ and Fourier expansion

$$
\psi(z)=\sum_{n \neq 0} b_{n} W_{0, s_{0}-\frac{1}{2}}(4 \pi|n| y) e(n x) .
$$

We will freely use that we can assume that the Fourier coefficients are real.

We want to study

$$
\int_{\Gamma \backslash \mathbb{\boxplus}} \psi(z)\left|E\left(z, s, \frac{1}{2}\right)\right|^{2} d \mu(z)
$$

when $\Re(s)=\frac{1}{2}$. It turns out to be convenient to consider the slightly more general integral

$$
I(s, w)=\int_{\Gamma \backslash \mathbb{\Psi}} \psi(z) E\left(z, w, \frac{1}{2}\right) \overline{E\left(z, \bar{s}, \frac{1}{2}\right)} d \mu(z) .
$$

For sufficiently large $\mathfrak{R}(s)$, we can unfold to get

$$
I(s, w)=\int_{\Gamma_{\infty} \backslash \mathbb{H}} \psi(z) E\left(z, w, \frac{1}{2}\right) y^{s} d \mu(z) .
$$

Using the Fourier expansions of $\psi$ and $E_{\infty}\left(z, w, \frac{1}{2}\right)$, and computing the $x$-integral, we find

$$
\begin{aligned}
I(s, w) & =\int_{0}^{\infty} \sum_{n \neq 0} b_{n} \phi_{-n}\left(w, \frac{1}{2}\right) W_{0, s_{0}-\frac{1}{2}}(4 \pi|n| y) W_{-\frac{1}{4} n /|n|, w-\frac{1}{2}}(4 \pi|n| y) y^{s-1} \frac{d y}{y} \\
& =\sum_{n \neq 0} \frac{b_{n} \phi_{-n}\left(w, \frac{1}{2}\right)}{(2 \pi|n|)^{s-1}} \int_{0}^{\infty} W_{0, s_{0}-\frac{1}{2}}(2 y) W_{-\frac{1}{4} n /|n|, w-\frac{1}{2}}(2 y) y^{s-1} \frac{d y}{y} . \quad \text { (4-2) }
\end{aligned}
$$

We consider the series

$$
Z_{ \pm}(s, w):=\frac{\Gamma\left(w \mp \frac{1}{4}\right)}{\pi^{w} e^{-i \pi / 4}} \zeta_{2}(4 s-1) \zeta_{2}(4 w-1) \sum_{ \pm n=1}^{\infty} \frac{b_{n} \phi_{-n}\left(w, \frac{1}{2}\right)}{|n|^{s-1}}
$$


By (3-3) we see that

$$
Z_{ \pm}(s, w)=\zeta_{2}(4 s-1) \sum_{ \pm n=1}^{\infty} \frac{b_{n} r_{2}(w,-n) L^{*}\left(2 w-\frac{1}{2},-n, 1\right)}{|n|^{s-w}}
$$

The next proposition reduces many questions about $Z_{ \pm}(s, w)$ to questions about $Z\left(s, w, \chi, \chi^{\prime}\right)$. Consider the Dirichlet polynomial

$$
T(s, w):=\prod_{\epsilon \in\{ \pm 1\}} p_{2}\left(\epsilon 2^{-\left(s+w-\frac{1}{2}\right)}\right) p_{2}\left(\epsilon 2^{-\left(s-w+\frac{1}{2}\right)}\right),
$$

where $p_{2}(z)$ is defined in (2-24).

Proposition 4.1. There exist functions $f_{ \pm}\left(s, w, \chi, \chi^{\prime}\right)$ bounded in vertical strips such that

$$
T(s, w) Z_{ \pm}(s, w)=\sum_{\chi, \chi^{\prime}} f_{ \pm}\left(s, w, \chi, \chi^{\prime}\right) Z\left(s, w, \chi, \chi^{\prime}\right)
$$

where the sum is over all pairs of characters mod 8.

Proof. We first assume that $\psi$ is a newform. Then we have

$$
b_{n}=b_{n /|n|}|n|^{-\frac{1}{2}} t_{|n|},
$$

where $\left\{t_{n}\right\}_{n \in \mathbb{N}}$ are the coefficients of $L(s, \psi)$. We note that if $m \geq 1$ is odd then $\chi_{\left( \pm 2^{l} m\right)_{0}}=\chi_{m_{0}} \chi$ where $m_{0}$ denotes the squarefree part of $m$ for some character $\chi$ whose conductor divides 8 , namely

$$
\chi(d)= \begin{cases}\left(\frac{ \pm 2}{d}\right) & \text { if } l \text { odd } \\ \left(\frac{ \pm 1}{d}\right) & \text { if } l \text { even }\end{cases}
$$

Notice that $\chi$ depends only on $l \bmod 2$ and the sign \pm . For the same $\chi$ we have $q(w, m, \chi)=q\left(w, \pm 2^{l} m\right)$. It follows that $L^{*}(s, m, \chi)=L^{*}\left(s, \pm 2^{l} m, 1\right)$. We write the summation index $n$ in (4-3) as $n=2^{l} m$, where $m$ is odd, and split the sum as

$$
\sum_{\substack{l=0 \\ l \text { odd }}}^{\infty} \sum_{\substack{ \pm m=1 \\(m, 2)=1}}^{\infty} \cdots+\sum_{\substack{l=0 \\ l \text { even }}}^{\infty} \sum_{\substack{ \pm m=1 \\(m, 2)=1}}^{\infty} \cdots
$$

We split the $m$ sum further according to $m \equiv 1,3,5,7(\bmod 8)$, which can be done by using a linear combination of characters. We then use the explicit formulae for $r_{2}(w,-n)$ in (3-6), (3-7) and that the Fourier coefficients satisfy the Hecke relations to see that $Z_{ \pm}(s, w)$ can be written as a linear combination of $Z\left(s, w, \chi, \chi^{\prime}\right)$ with coefficients being functions bounded on vertical strips multiplied by one of the following series: 


$$
\begin{array}{cc}
\sum_{j=0}^{\infty} \frac{t_{2^{2 j}}}{2^{2 j\left(s+w-\frac{1}{2}\right)}}, & \sum_{j=0}^{\infty} \frac{t_{2^{2 j+1}}}{2^{(2 j+1)\left(s+w-\frac{1}{2}\right)}}, \\
\sum_{j=0}^{\infty} \frac{t_{2^{2 j} u_{j}\left(2^{-(2 w-1)}\right)}}{2^{2 j\left(s-w+\frac{1}{2}\right)}}, & \sum_{j=0}^{\infty} \frac{t_{2^{2 j+1} u_{j}\left(2^{-(2 w-1)}\right)}}{2^{(2 j+1)\left(s-w+\frac{1}{2}\right)}} .
\end{array}
$$

We easily see that

$$
2 \sum_{j=0}^{\infty} \frac{t_{2^{2 j}}}{2^{2 j s}}=\frac{1}{p_{2}\left(2^{-s}\right)}+\frac{1}{p_{2}\left(-2^{-s}\right)}, \quad 2 \sum_{j=0}^{\infty} \frac{t_{2^{2 j+1}}}{2^{(2 j+1) s}}=\frac{1}{p_{2}\left(2^{-s}\right)}-\frac{1}{p_{2}\left(-2^{-s}\right)} .
$$

We see also that, using (3-8),

$$
\sum_{j=0}^{\infty} \frac{t_{2^{2 j}} u_{j}(x)}{2^{2 j s}}=\frac{x^{2}}{2\left(1-x^{2}\right)}\left(\frac{1}{p_{2}\left(2^{-s}\right)}+\frac{1}{p_{2}\left(-2^{-s}\right)}-\frac{1}{p_{2}\left(x 2^{-s}\right)}-\frac{1}{p_{2}\left(-x 2^{-s}\right)}\right)
$$

which has no poles coming out of $x^{2}-1$ in the denominator. Similarly, we see that $\sum_{j=0}^{\infty} \frac{t_{2^{2 j+1}} u_{j}(x)}{2^{(2 j+1) s}}=\frac{x^{2}}{2\left(1-x^{2}\right)}\left(\frac{1}{p_{2}\left(2^{-s}\right)}-\frac{1}{p_{2}\left(-2^{-s}\right)}-\frac{1}{x}\left(\frac{1}{p_{2}\left(x 2^{-s}\right)}-\frac{1}{p_{2}\left(-x 2^{-s}\right)}\right)\right)$.

We substitute in the last four equations $s+w-\frac{1}{2}$ or $s-w+\frac{1}{2}$ for $s$ as required and $x=2^{-(2 w-1)}$ to identify the possible polynomials that appear in the denominators. These have product $T(s, w)$. We now notice that multiplying any of the 4 functions in (4-5) by $T(s, w)$ we get holomorphic functions bounded on vertical strips, which proves the claim.

If $\psi$ is an oldform with, say, $\psi=\psi_{1}\left(2^{j} z\right)$ with $\psi_{1}$ a primitive form, and $j=1,2$, then the series in (4-2) becomes

$$
\sum_{n \neq 0} \frac{b_{n}\left(\psi_{1}\right) \phi_{-2^{j} n}\left(w, \frac{1}{2}\right)}{\left(2 \pi\left|2^{j} n\right|\right)^{s-1}}
$$

which by the explicit expression for $\phi_{n}\left(w, \frac{1}{2}\right)$ can be analyzed similarly to the newform case.

Remark 4.2. In Theorem 4.3 below, we need to study $Z_{ \pm}\left(\frac{1}{2}+i t, \frac{1}{2}-i t\right)$. For $\mathfrak{R}(s)=\mathfrak{R}(w)=\frac{1}{2}$ we notice that by $(2-25)$ we have $1 / T(s, w)=O(1)$.

Theorem 4.3. Assume that for any $\chi, \chi^{\prime} \bmod 8$ the function $Z\left(s, 1-s, \chi, \chi^{\prime}\right)$ satisfies a subconvex bound. Then

$$
\int_{\Gamma \backslash \mathbb{M}} \psi(z)\left|E\left(z, \frac{1}{2}+i t, \frac{1}{2}\right)\right|^{2} d \mu(z) \rightarrow 0 \quad \text { as }|t| \rightarrow \infty .
$$


Proof. By Proposition 4.1, a subconvex bound with saving $\delta$ translates into a bound $Z_{ \pm}(s, 1-s)=O\left(|t|^{2\left(\frac{1}{4}-\delta\right)}\right)$ when $\Re(s)=\frac{1}{2}$. Combining this with the bound in Lemma A.1, the estimate $1 / \zeta(1+i t)=O(\log |t|)$ [Titchmarsh 1986, Equation 3.11.8], and the identity (4-2) we see that $I(s, 1-s)=O\left(|t|^{2\left(\frac{1}{4}-\delta\right)-\frac{1}{2}+\varepsilon}\right)$ for any $\varepsilon>0$ when $\Re(s)=\frac{1}{2}$. Since

$$
I\left(\frac{1}{2}+i t, \frac{1}{2}-i t\right)=\int_{\Gamma \backslash \boxplus} \psi(z)\left|E\left(z, \frac{1}{2}-i t, \frac{1}{2}\right)\right|^{2} d \mu(z),
$$

we find that, when $\delta>0, I\left(\frac{1}{2}+i t, \frac{1}{2}-i t\right) \rightarrow 0$ as $|t| \rightarrow \infty$.

Remark 4.4. In the proof above we see that the trivial bound from Theorem 2.17 only gives $O\left(|t|^{\frac{1}{2}+\varepsilon}\right)$.

4B. The incomplete Eisenstein series contribution. In the following we choose a fundamental domain of $\Gamma$ such that

$$
\mathscr{D}=\mathscr{D}_{0} \cup \bigcup_{j=1}^{3} \sigma_{\mathfrak{a}_{j}} \mathscr{D}^{Y}
$$

where $\mathscr{D}^{Y}:=\{x+i y: 0<x<1, y>Y\}, Y$ sufficiently large, $\mathscr{D}_{0}$ is a suitable compact set and, as before, $\sigma_{\mathfrak{a}_{j}}$ denotes the scaling matrix of the cusp $\mathfrak{a}_{j}$.

In order to introduce the incomplete Eisenstein series, let $h(y) \in C^{\infty}\left(\mathbb{R}^{+}\right)$be a function which decreases rapidly at 0 and $\infty$, and whose derivatives are also of rapid decay. Its Mellin transform evaluated at $-s$ is

$$
H(s)=\int_{0}^{\infty} h(y) y^{-s} \frac{d y}{y}
$$

and thus by the Mellin inversion formula we have

$$
h(y)=\frac{1}{2 \pi i} \int_{\Re s=a} H(s) y^{s} d s
$$

for any $a \in \mathbb{R}$. The function $H(s)$ is entire and $H(a+i t)$ is in the Schwartz space in the $t$ variable for any $a \in \mathbb{R}$. The incomplete Eisenstein series corresponding to the cusp $\mathfrak{a}$ is then given by

$$
F_{h}(z, \mathfrak{a})=\sum_{\gamma \in \Gamma_{\mathfrak{a}} \backslash \Gamma} h\left(\Im \sigma_{\mathfrak{a}}^{-1} \gamma z\right)=\frac{1}{2 \pi i} \int_{\Re s=a>1} H(s) E_{\mathfrak{a}}(z, s, 0) d s .
$$

For $i=1,2,3$ we are interested in the behavior of

$$
J\left(t, \mathfrak{a}_{i}\right)=\int_{\Gamma \backslash \mathbb{\sharp}} F_{h}\left(z, \mathfrak{a}_{i}\right)\left|E\left(z, \frac{1}{2}+i t, \frac{1}{2}\right)\right|^{2} d \mu(z) \quad \text { as }|t| \rightarrow \infty .
$$

In the following we only treat the contribution from the cusp at infinity, but the other contributions can be dealt with similarly. Unfolding the incomplete Eisenstein 
series we find

$$
J(t, \infty)=\int_{\Gamma \backslash \mathbb{M}} F_{h}(z, \infty) d \mu_{t}(z)=J_{1}(t, \infty)+J_{2}(t, \infty)
$$

with

$$
\begin{aligned}
& J_{1}(t, \infty):=\int_{0}^{\infty} h(y)\left|y^{\frac{1}{2}+i t}+\phi\left(\frac{1}{2}+i t, \frac{1}{2}\right) y^{\frac{1}{2}-i t}\right|^{2} \frac{d y}{y^{2}}, \\
& J_{2}(t, \infty):=\int_{0}^{\infty} h(y) \sum_{n \neq 0}\left|\phi_{n}\left(\frac{1}{2}+i t, \frac{1}{2}\right)\right|^{2}\left|W_{n /(4|n|), i t}(4 \pi|n| y)\right|^{2} \frac{d y}{y^{2}} .
\end{aligned}
$$

The integral $J_{1}(t, \infty)$ is easily dealt with. Namely, we obtain

$$
\begin{aligned}
& J_{1}(t, \infty) \\
& =\left(1+\left|\phi\left(\frac{1}{2}+i t, \frac{1}{2}\right)\right|^{2}\right) \int_{0}^{\infty} h(y) \frac{d y}{y}+\phi\left(\frac{1}{2}+i t, \frac{1}{2}\right) \int_{0}^{\infty} h(y) y^{-2 i t} \frac{d y}{y} \\
& =\left(1+\left|\phi\left(\frac{1}{2}+i t, \frac{1}{2}\right)\right|^{2}\right) H(0)+\phi\left(\frac{1}{2}+i t, \frac{1}{2}\right) H(2 i t)+\overline{\phi\left(\frac{1}{2}+i t, \frac{1}{2}\right)} \int_{0}^{\infty} h(y) y^{2 i t} \frac{d y}{y} \\
& =O(1) .
\end{aligned}
$$

For the integral $J_{2}(t, \infty)$ we find, using the rapid decay of the Whittaker function and the Mellin inversion formula,

$$
J_{2}(t, \infty)=\frac{1}{2 \pi i} \int_{\Re s=a>1} H(s) R_{1}\left(\left|E\left(z, \frac{1}{2}+i t, \frac{1}{2}\right)\right|^{2}, s\right) d s,
$$

where

$$
\begin{aligned}
R_{1}\left(\left|E\left(z, w, \frac{1}{2}\right)\right|^{2}, s\right) & =\sum_{n \neq 0}\left|\phi_{n}\left(w, \frac{1}{2}\right)\right|^{2} \int_{0}^{\infty}\left|W_{n /(4|n|), w-\frac{1}{2}}(4 \pi|n| y)\right|^{2} y^{s-1} \frac{d y}{y} \\
& =\sum_{n \neq 0} \frac{\left|\phi_{n}\left(w, \frac{1}{2}\right)\right|^{2}}{(2 \pi|n|)^{s-1}} \int_{0}^{\infty}\left|W_{n /(4|n|), w-\frac{1}{2}}(2 y)\right|^{2} y^{s-1} \frac{d y}{y} .
\end{aligned}
$$

In order to analyze the asymptotic behavior of $J_{2}(t, \infty)$ we need to understand the function $R_{1}\left(\left|E\left(z, w, \frac{1}{2}\right)\right|^{2}, s\right)$. There are (at least) two ways to do this: to use properties of the double Dirichlet series we defined in Section 2, or to use Zagier's theory of the Rankin-Selberg method for functions that are not of rapid decay but satisfy a certain mild growth condition. We will actually use a combination of these two techniques. We want to shift the line of integration in (4-12) to $\Re(s)=\frac{1}{2}$. For this we need to identify the poles, estimate them, see what the contribution of $\int_{\mathfrak{R}(s)=\frac{1}{2}} H(s) R_{1}\left(\left|E\left(z, w, \frac{1}{2}\right)\right|^{2}, s\right)$ is to the asymptotics. For the first and third aspect we use the Rankin-Selberg approach and for the second aspect the multiple Dirichlet approach works best. 
We first describe why double Dirichlet series techniques apply. The growth of the Mellin transform of the absolute value of the Whittaker function is analyzed in Lemma A.5. By combining (3-3), (3-6), and (3-7) we see that $\overline{\phi_{n}\left(\bar{w}, \frac{1}{2}\right)}=\phi_{n}\left(w, \frac{1}{2}\right)$. This shows that when $\Re(w)=\frac{1}{2}$ we have

$$
\left|\phi_{n}\left(w, \frac{1}{2}\right)\right|^{2}=\phi_{n}\left(w, \frac{1}{2}\right) \phi_{n}\left(1-w, \frac{1}{2}\right) .
$$

The right-hand side has the advantage of being meromorphic in $w$. We define

$$
\widehat{Z}_{ \pm}(s, w)=\frac{\Gamma\left(w \pm \frac{1}{4}\right) \Gamma\left(1-w \pm \frac{1}{4}\right)}{\pi i} \sum_{ \pm n=1}^{\infty} \frac{\phi_{n}\left(w, \frac{1}{2}\right) \phi_{n}\left(1-w, \frac{1}{2}\right)}{|n|^{s-1}},
$$

which by (3-3) equals

$$
\begin{aligned}
& \frac{1}{\zeta_{2}(4 w-1) \zeta_{2}(4(1-w)-1)} \\
& \quad \times \sum_{ \pm n=1}^{\infty} \frac{L^{*}\left(2 w-\frac{1}{2}, n, 1\right) L^{*}\left(2(1-w)-\frac{1}{2}, n, 1\right)}{|n|^{s}} r_{2}(w, n) r_{2}(1-w, n) .
\end{aligned}
$$

We now show that $\widehat{Z}_{ \pm}(s, w)$ is directly related to the function $\widehat{Z}\left(s, w, \chi, \chi^{\prime}\right)$ defined in (2-40). Let

$$
U(s, w)=\left(1-2^{-(4 w-1)}\right)\left(1-2^{-2 s}\right)\left(1-2^{-(4 w-2+2 s)}\right)\left(1-2^{-(-4 w+2+2 s)}\right) .
$$

Proposition 4.5. There exist functions $\hat{f}_{ \pm, \kappa}\left(s, w, \chi, \chi^{\prime}\right)$ bounded in vertical strips such that

$$
\begin{aligned}
U(s, w) \widehat{Z}_{ \pm}(s, w)= & \frac{1}{\zeta_{2}(4 w-1) \zeta_{2}(4(1-w)-1)} \sum_{\kappa \in\{0,1\}} \frac{\Gamma\left(\frac{1}{2}\left(2 w-\frac{1}{2}+\kappa\right)\right)}{\Gamma\left(\frac{1}{2}\left(2(1-w)-\frac{1}{2}+\kappa\right)\right)} \\
& \times \sum_{\chi, \chi^{\prime}} \hat{f}_{ \pm, \kappa}\left(s, w, \chi, \chi^{\prime}\right) \widehat{Z}\left(\frac{s+2 w-\frac{1}{2}}{2}, \frac{s-2 w+\frac{3}{2}}{2}, \chi, \chi^{\prime}\right) .
\end{aligned}
$$

Proof. As in the proof of Proposition 4.1 we write $n=2^{l} m$ and split into sums over $l$ even, odd respectively. We then split the $m$ sum according to the residue class mod 8 which is a linear combination over characters mod 8 . Inserting the explicit formulae for $r_{2}(w, n),(3-6),(3-7)$ we are led to consider the series

$$
\sum_{j=0}^{\infty} u_{j}(x) u_{j}(y) z^{j}, \quad \sum_{j=0}^{\infty} u_{j}(x) z^{j}, \quad \sum_{j=0}^{\infty} z^{j}
$$

with $x, y, z$ being appropriate powers of 2 . Since these are all sums of geometric series - see (3-8) - they are explicitly computable and after multiplying by 
$\left(1-2^{-2 s}\right)\left(1-2^{-(4 w-2+2 s)}\right)\left(1-2^{-(-4 w+2+2 s)}\right)$ they become Dirichlet polynomials in powers of 2, hence holomorphic and bounded in vertical strips. Therefore

$$
\begin{aligned}
& \left(1-2^{-2 s}\right)\left(1-2^{-(4 w-2+2 s)}\right)\left(1-2^{-(-4 w+2+2 s)}\right) \widehat{Z}_{ \pm}(s, w) \\
& =\sum_{\chi, \chi^{\prime}} \tilde{f}_{ \pm}\left(s, w, \chi, \chi^{\prime}\right) \widetilde{Z}\left(s, w, \chi, \chi^{\prime}\right),
\end{aligned}
$$

where

$$
\begin{aligned}
& \widetilde{Z}\left(s, w, \chi, \chi^{\prime}\right) \\
& =\frac{1}{\zeta_{2}(4 w-1) \zeta_{2}(4(1-w)-1)} \sum_{n=1}^{\infty} \frac{\chi^{\prime}(n) L^{*}\left(2 w-\frac{1}{2}, n, \chi\right) L^{*}\left(2(1-w)-\frac{1}{2}, n, \chi\right)}{n^{s}}
\end{aligned}
$$

and $\tilde{f}_{ \pm}\left(s, w, \chi, \chi^{\prime}\right)$ are bounded in vertical strips. Using the functional equation on $L^{*}\left(2(1-w)-\frac{1}{2}, n, \chi\right)$ we see - as in the proof of Theorem 2.11 - that

$$
\begin{aligned}
\left(1-2^{-(4 w-1)}\right) & \sum_{n=1}^{\infty} \frac{\chi^{\prime}(n) L^{*}\left(2 w-\frac{1}{2}, n, \chi\right) L^{*}\left(2(1-w)-\frac{1}{2}, n, \chi\right)}{n^{s}} \\
& =\sum_{\kappa \in\{0,1\}} \frac{\Gamma\left(\frac{1}{2}\left(2 w-\frac{1}{2}+\kappa\right)\right)}{\Gamma\left(\frac{1}{2}\left(2(1-w)-\frac{1}{2}+\kappa\right)\right)} \sum_{\chi, \chi^{\prime}} \tilde{\tilde{f}}_{\kappa}\left(x, y, \chi, \chi^{\prime}\right) \widetilde{Z}\left(s, w, \chi, \chi^{\prime}\right),
\end{aligned}
$$

where $\tilde{\tilde{f}}_{\kappa}\left(x, y, \chi, \chi^{\prime}\right)$ is another set of functions bounded in vertical strips and

$$
\widetilde{Z}\left(s, w, \chi, \chi^{\prime}\right)=\sum_{n=1}^{\infty} \frac{\chi^{\prime}(n) L^{*}\left(2 w-\frac{1}{2}, n, \chi\right)^{2}}{n^{s-2 w+1}} .
$$

Combining the above equations and comparing with (2-40) finishes the proof.

The above lemma implies that many questions about $R_{1}\left(\left|E\left(z, w, \frac{1}{2}\right)\right|^{2}, s\right)$ can be dealt with using $Z\left(s, w, \chi, \chi^{\prime}\right)$. We now describe a different method for understanding $R_{1}\left(\left|E\left(z, w, \frac{1}{2}\right)\right|^{2}, s\right)$, namely Zagier's Rankin-Selberg method for functions not of rapid decay. This method was introduced by Zagier [1981] for the group $\mathrm{SL}_{2}(\mathbb{Z})$ and generalized by Kudla (unpublished), Dutta Gupta [1997], and Mizuno [2005]. Its usefulness for determining the contribution of the incomplete Eisenstein series to the asymptotics can already be seen in [Zelditch 1991]. We introduce the generalized Rankin-Selberg transform, following [Zagier 1981] and [Mizuno 2005]. We write $e_{i j}(y, s, k)=\delta_{i j} y^{s}+\phi_{i j}(s, k) y^{1-s}$ for the zero Fourier coefficient of $E_{\mathfrak{a}_{i}}(z, s, k)$ at $\mathfrak{a}_{j}$ and we denote the scattering matrix by $\Phi(s, k)=\left(\phi_{i j}(s, k)\right)$. We note that for $\Gamma_{0}(4)$ the matrix $\Phi(s, 0)$ is $3 \times 3$ whereas $\Phi\left(s, \frac{1}{2}\right)$ is $2 \times 2$. For the weight 0 Eisenstein series we use the notation $E_{i}(z, s, 0)=E_{\mathfrak{a}_{i}}(z, s, 0)$. 
Theorem 4.6 [Mizuno 2005, Theorem 2]. Let $F$ be a continuous functions on $\mathbb{W}$ that is $\Gamma$-invariant and satisfies, for $i=1,2,3$,

$$
F\left(\sigma_{\mathfrak{a}_{i}} z\right)=\psi_{i}(y)+O\left(y^{-N}\right) \text { for all } N \text { as } y \rightarrow \infty,
$$

where

$$
\psi_{i}(y)=\sum_{j=1}^{l} \frac{c_{i j}}{n_{i j} !} y^{\alpha_{i j}} \log ^{n_{i j}} y, \quad n_{i j} \in \mathbb{N} \cup\{0\}, \quad i=1,2,3 .
$$

For such a function $F$ the Rankin-Selberg transform $R_{i}(F, s)$ corresponding to the cusp $\mathfrak{a}_{i}, i=1,2,3$, is defined by

$$
R_{i}(F, s):=\int_{0}^{\infty} \int_{0}^{1}\left(F\left(\sigma_{\mathfrak{a}_{i}} z\right)-\psi_{i}(y)\right) y^{s} d \mu(z)
$$

for $\Re$ s sufficiently large. Then we have

$$
\begin{aligned}
& R_{i}(F, s)= \int_{\mathscr{D}_{0}} F(z) E_{i}(z, s, 0) d \mu(z) \\
&+\sum_{j=1}^{3} \int_{\mathscr{D}^{Y}}\left(F\left(\sigma_{\mathfrak{a}_{j}} z\right) E_{i}\left(\sigma_{\mathfrak{a}_{j}} z, s, 0\right)-\psi_{j}(y) e_{i j}(y, s, 0)\right) d \mu(z) \\
& \quad+\sum_{j=1}^{3} \phi_{i j}(s, 0) \int_{Y}^{\infty} \psi_{j}(y) y^{-s-1} d y-\int_{0}^{Y} \psi_{i}(y) y^{s-2} d y \\
&= \int_{\mathscr{D}_{0}} F(z) E_{i}(z, s, 0) d \mu(z) \\
&+\sum_{j=1}^{3} \int_{\mathscr{D}^{Y}}\left(F\left(\sigma_{\mathfrak{a}_{j}} z\right) E_{i}\left(\sigma_{\mathfrak{a}_{j}} z, s, 0\right)-\psi_{j}(y) e_{i j}(y, s, 0)\right) d \mu(z) \\
& \quad-\sum_{j=1}^{3} \phi_{i j}(s, 0) \widehat{\psi_{j}}(1-s, Y)-\widehat{\psi}_{i}(s, Y),
\end{aligned}
$$

where

$$
\widehat{\psi}_{i}(s, Y)=\sum_{j=1}^{l} c_{i j} \sum_{m=0}^{n_{i j}} \frac{(-1)^{n_{i j}-m}}{m !} \frac{Y^{s+\alpha_{i j}-1} \log ^{m} Y}{\left(s+\alpha_{i j}-1\right)^{n_{i j}-m+1}} .
$$

Furthermore, for each $i=1,2,3$, the function $R_{i}(F, s)$ can be meromorphically continued to $\mathbb{C}$ and we have the functional equation

$$
\mathscr{R}(F, s):={ }^{t}\left(R_{1}(F, s), R_{2}(F, s), R_{3}(F, s)\right)=\Phi(s, 0) \mathscr{R}(F, 1-s) .
$$

We want to move the line of integration in (4-12) to $\Re(s)=\frac{1}{2}$ and Theorem 4.6 plays a major role, as it allows to identify the relevant poles and to calculate the corresponding residues. By the above theorem, in particular by (4-14), we infer 


$$
\begin{aligned}
& R_{1}\left(\left|E\left(z, \frac{1}{2}+i t, \frac{1}{2}\right)\right|^{2}, s\right) \\
& \left.=\int_{\mathscr{D}_{0}}\left|E\left(z, \frac{1}{2}+i t, \frac{1}{2}\right)\right|^{2} E_{1}(z, s, 0) d \mu(z)-\widehat{\psi(s, Y}\right) \\
& +\sum_{j=1}^{3} \int_{\mathscr{D}^{Y}}\left(\left|E\left(\sigma_{\mathfrak{a}_{j}} z, \frac{1}{2}+i t, \frac{1}{2}\right)\right|^{2} E_{1}\left(\sigma_{\mathfrak{a}_{j}} z, s, 0\right)-\psi_{j}(y) e_{1 j}(y, s, 0)\right) d \mu(z),
\end{aligned}
$$

where

$$
\begin{aligned}
\widehat{\psi(s, Y}) & =\widehat{\psi_{1}}(s, Y)+\phi_{11}(s, 0) \widehat{\psi_{1}}(1-s, Y)+\frac{Y^{1-s}}{1-s} \phi_{12}(s, 0)\left|\phi_{12}\left(\frac{1}{2}+i t, \frac{1}{2}\right)\right|^{2}, \\
\widehat{\psi_{1}}(s, Y) & =\frac{Y^{s}}{s}\left(1+\left|\phi_{11}\left(\frac{1}{2}+i t, \frac{1}{2}\right)\right|^{2}\right)+\frac{Y^{s-2 i t}}{s-2 i t} \phi_{11}\left(\frac{1}{2}+i t, \frac{1}{2}\right)+\frac{Y^{s+2 i t}}{s+2 i t} \overline{\phi_{11}\left(\frac{1}{2}+i t, \frac{1}{2}\right)}, \\
\psi_{j}(y) & =\left|\delta_{1 j} y^{\frac{1}{2}+i t}+\phi_{1 j}\left(\frac{1}{2}+i t, \frac{1}{2}\right) y^{\frac{1}{2}-i t}\right|^{2}, \quad j=1,2, \\
\psi_{3}(y) & =0 .
\end{aligned}
$$

Thus we easily see that we pick up residues at $s=1$ and $s=1 \pm 2 i t$ when we shift the line of integration. The pole at $s=1$ is responsible for the contribution of the $\log |t|$ term in (1-10), as we will see. We therefore examine $H(s) R_{1}\left(\left|E\left(z, \frac{1}{2}+i t, \frac{1}{2}\right)\right|^{2}, s\right)$ at $s=1$. In order to determine the order of the pole at $s=1$ and its residue we use the Laurent expansion of $H(s)$ and $R_{1}\left(\left|E\left(z, \frac{1}{2}+i t, \frac{1}{2}\right)\right|^{2}, s\right)$. The first two terms of (4-15) are easily understood because of the Eisenstein series, which has simple poles at $s=1$ and no other poles in $\Re(s) \geq \frac{1}{2}$. In order to treat the last term of (4-15) we write

$$
\begin{aligned}
\frac{Y^{1-s}}{1-s} & =-\frac{1}{s-1}+\log Y+O(|s-1|), \\
\phi_{1 j}(s, 0) & =\frac{1}{\operatorname{vol}(\Gamma \backslash \mathbb{W})} \frac{1}{s-1}+b_{0}^{1 j}+O(|s-1|) .
\end{aligned}
$$

These expansions and the fact that the scattering matrix $\Phi\left(s, \frac{1}{2}\right)=\left(\phi_{i j}\left(s, \frac{1}{2}\right)\right)_{1 \leq i, j \leq 2}$ is unitary for $\Re(s)=\frac{1}{2}$ (see [Roelcke 1966, Lemma 10.5]) yield

$$
\begin{aligned}
& \widehat{\psi(s, Y)} \\
& =-\frac{1}{\operatorname{vol}(\Gamma \backslash \mathbb{W})}\left(1+\sum_{j=1}^{2}\left|\phi_{1 j}\left(\frac{1}{2}+i t, \frac{1}{2}\right)\right|^{2}\right) \frac{1}{(s-1)^{2}} \\
& +\left(\left(1+\sum_{j=1}^{2}\left|\phi_{1 j}\left(\frac{1}{2}+i t, \frac{1}{2}\right)\right|^{2}\right) \frac{\log Y}{\operatorname{vol}(\Gamma \backslash \mathbb{U})}\right. \\
& -\left(1+\left|\phi_{11}\left(\frac{1}{2}+i t, \frac{1}{2}\right)\right|^{2}\right) b_{0}^{11}-\left|\phi_{12}\left(\frac{1}{2}+i t, \frac{1}{2}\right)\right|^{2} b_{0}^{12}
\end{aligned}
$$

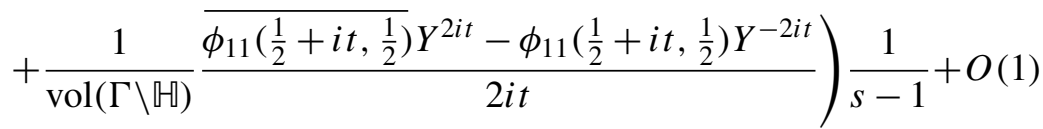




$$
\begin{aligned}
=- & \frac{2}{\operatorname{vol}(\Gamma \backslash \mathbb{W})} \frac{1}{(s-1)^{2}} \\
& +\left(\frac{2 \log Y}{\operatorname{vol}(\Gamma \backslash \mathbb{U})}-\left(1+\left|\phi_{11}\left(\frac{1}{2}+i t, \frac{1}{2}\right)\right|^{2}\right) b_{0}^{11}-\left|\phi_{12}\left(\frac{1}{2}+i t, \frac{1}{2}\right)\right|^{2} b_{0}^{12}\right. \\
& \left.\quad+\frac{1}{\operatorname{vol}(\Gamma \backslash \mathbb{W})} \frac{\frac{\phi_{11}\left(\frac{1}{2}+i t, \frac{1}{2}\right)}{2} Y^{2 i t}-\phi_{11}\left(\frac{1}{2}+i t, \frac{1}{2}\right) Y^{-2 i t}}{2 i t}\right) \frac{1}{s-1}+O(1) .
\end{aligned}
$$

Consequently we see that $R_{1}\left(\left|E\left(z, \frac{1}{2}+i r, \frac{1}{2}\right)\right|^{2}, s\right)$ has a pole of order 2 in $s=1$. Furthermore,

$$
\begin{aligned}
& \operatorname{res}_{s=1} H(s) R_{1}\left(\left|E\left(z, \frac{1}{2}+i t, \frac{1}{2}\right)\right|^{2}, s\right) \\
& =\left(\frac { 1 } { \operatorname { v o l } ( \Gamma \backslash \mathbb { U } ) } \left(-2 \log Y+\int_{\mathscr{D}_{0}}\left|E\left(z, \frac{1}{2}+i t, \frac{1}{2}\right)\right|^{2} d \mu(z)\right.\right. \\
& +\sum_{j=1}^{3} \int_{\mathscr{D}^{Y}}\left(\left|E\left(\sigma_{\mathfrak{a}_{j}} z, \frac{1}{2}+i t, \frac{1}{2}\right)\right|^{2}-\psi_{j}(y)\right) d \mu(z) \\
& \left.-\frac{\overline{\phi_{11}\left(\frac{1}{2}+i t, \frac{1}{2}\right)} Y^{2 i t}-\phi_{11}\left(\frac{1}{2}+i t, \frac{1}{2}\right) Y^{-2 i t}}{2 i t}\right) \\
& \left.+b_{0}^{11}+\sum_{j=1}^{2}\left|\phi_{1 j}\left(\frac{1}{2}+i t, \frac{1}{2}\right)\right|^{2} b_{0}^{1 j}\right) H(1)+\frac{2 H^{\prime}(1)}{\operatorname{vol}(\Gamma \backslash \mathbb{U})} \\
& =\left(-\frac{1}{\operatorname{vol}(\Gamma \backslash \mathbb{U})} \sum_{j=1}^{2} \phi_{1 j}^{\prime}\left(\frac{1}{2}+i t, \frac{1}{2}\right) \overline{\phi_{1 j}\left(\frac{1}{2}+i t, \frac{1}{2}\right)}\right. \\
& \left.+b_{0}^{11}+\sum_{j=1}^{2}\left|\phi_{1 j}\left(\frac{1}{2}+i t, \frac{1}{2}\right)\right|^{2} b_{0}^{1 j}\right) H(1)+\frac{2 H^{\prime}(1)}{\operatorname{vol}(\Gamma \backslash \mathbb{U})},
\end{aligned}
$$

where we used the Maaß-Selberg relations (see [Roelcke 1966, Lemma 11.2], for example). For the remaining poles at $s=1 \pm 2 i t$ we obtain

$$
\text { res } H(s) R_{1}\left(\left|E\left(z, \frac{1}{2}+i t, \frac{1}{2}\right)\right|^{2}, s\right)=H(1+2 i t) \phi_{11}(1+2 i t, 0) \overline{\phi_{11}\left(\frac{1}{2}+i t, \frac{1}{2}\right)},
$$

and this expression is of rapid decay as $|t| \rightarrow \infty$. This follows from the following general facts: the entries of the scattering matrix of weight zero are uniformly bounded for $\Re(s) \geq \frac{1}{2},|\Im(s)| \geq 1$ (see [Selberg 1989, p. 655], for example), $\phi_{11}\left(\frac{1}{2} \pm i t, \frac{1}{2}\right)$ is bounded since $\Phi\left(\frac{1}{2}+i t, k\right)$ is unitary, and we have the rapid decay of $H(1 \pm 2 i t)$. The same bound holds for the residue of $H(s) R_{1}\left(\left|E\left(z, \frac{1}{2}+i t, \frac{1}{2}\right)\right|^{2}, s\right)$ at $s=1-2 i t$. We now want to shift the line of integration in (4-12). To do this we need to control the growth of the $R_{1}\left(\left|E\left(z, \frac{1}{2}+i t, \frac{1}{2}\right)\right|^{2}, s\right)$ as well as knowing the residues. 
Lemma 4.7. Let $F(z)=\left|E_{1}\left(z, \frac{1}{2}+i t, \frac{1}{2}\right)\right|^{2}$. The function $R_{1}(F(z), \sigma+i v)$ is of at most polynomial growth as $|v| \rightarrow \infty$ for $\sigma \geq \frac{1}{2}$.

Proof. In order to avoid the poles of the Eisenstein series coming from the zeros of the zeta function in the critical strip we work with $R_{i}^{*}(F, s):=\zeta(2 s) R_{i}(F, s)$, $i=1,2,3$. Then the function $R_{i}^{*}(F, s)$ has only finitely many poles in the strip $0 \leq \Re(s) \leq 1$. The estimates for the Eisenstein series and the scattering matrix imply that

$$
R_{i}^{*}(F, s)=O(1)
$$

as $|\Im(s)| \rightarrow \infty$ for $\Re(s)>1, i=1,2,3$. Using the functional equation as well as explicit expressions for $\phi_{1 j}(s, 0)$ we then get

$$
R_{1}^{*}(F, s)=\frac{\zeta(2 s)}{\zeta(2(1-s))} \sum_{j=1}^{3} \phi_{1 j}(s, 0) R_{j}^{*}(F, 1-s)=O\left(|\Im(s)|^{1-2 \sigma}\right)
$$

as $|\Im(s)| \rightarrow \infty$ for $\sigma=\Re(s)<0, i=1,2,3$. Thus by the Phragmén-Lindelöf principle we finally obtain that

$$
R_{1}(F, \sigma+i v)=O\left(|v|^{k}\right) \quad \text { as }|v| \rightarrow \infty, \sigma \geq \frac{1}{2}, \text { for some } k \in \mathbb{N} .
$$

Now that polynomial growth has been established it follows from (4-16) that

$$
\begin{aligned}
J_{2}(t, \infty)=(- & \frac{1}{\operatorname{vol}(\Gamma \backslash \mathbb{W})} \sum_{j=1}^{2} \frac{\phi_{1 j}^{\prime}}{\phi_{1 j}}\left(\frac{1}{2}+i t, \frac{1}{2}\right)\left|\phi_{1 j}\left(\frac{1}{2}+i t, \frac{1}{2}\right)\right|^{2}+b_{0}^{11} \\
& \left.+\sum_{j=1}^{2}\left|\phi_{1 j}\left(\frac{1}{2}+i t, \frac{1}{2}\right)\right|^{2} b_{0}^{1 j}\right) H(1)+\frac{H^{\prime}(1)}{\pi} \\
& +\frac{1}{2 \pi i} \int_{\Re s=\frac{1}{2}} H(s) R_{1}\left(\left|E\left(z, \frac{1}{2}+i t, \frac{1}{2}\right)\right|^{2}, s\right) d s+O(1) .
\end{aligned}
$$

In Section 3 we saw that, up to constants and fractions of polynomials in powers of 2 , the entries of the scattering matrix are equal to $\xi(3-4 s) / \xi(4 s-1)$; see (3-10). Hence, in order to determine the asymptotic behavior of the first term in (4-17) with respect to the $t$-variable, we need to understand the logarithmic derivative of $\xi(3-4 s) / \xi(4 s-1)$ at $s=\frac{1}{2}+i t$. The contribution from the remaining terms is $O(1)$. We have

$$
\begin{aligned}
\left.\left(\log \frac{\xi(3-4 s)}{\xi(4 s-1)}\right)^{\prime}\right|_{s=\frac{1}{2}+i t}= & 4 \log \pi-2 \frac{\Gamma^{\prime}}{\Gamma}\left(\frac{1}{2}-2 i t\right)-2 \frac{\Gamma^{\prime}}{\Gamma}\left(\frac{1}{2}+2 i t\right) \\
& \quad-4\left(\frac{\zeta^{\prime}}{\zeta}(1-4 i t)-\frac{1}{4 i t}+\frac{\zeta^{\prime}}{\zeta}(1+4 i t)+\frac{1}{4 i t}\right) \\
= & -4 \log |t|+o(\log |t|)
\end{aligned}
$$


by Stirling's formula and [Titchmarsh 1986, Theorem 5.17]. Since $\Phi\left(s, \frac{1}{2}\right)$ is unitary for $\Re s=\frac{1}{2}$, we finally arrive at

$$
J_{2}(t, \infty)=\frac{4 H(1)}{\operatorname{vol}(\Gamma \backslash \mathbb{W})} \log |t|+\frac{1}{2 \pi i} \int_{\Re s=\frac{1}{2}} H(s) R_{1}\left(\left|E\left(z, \frac{1}{2}+i t, \frac{1}{2}\right)\right|^{2}, s\right) d s+o(\log |t|)
$$

as $|t| \rightarrow \infty$. To treat the last integral we use again the connection to double Dirichlet series.

Lemma 4.8. Assume that for any $\chi, \chi^{\prime} \bmod 8$ the function $Z_{\psi_{\tau}}\left(s, 1-s, \chi, \chi^{\prime}\right)$ satisfies a subconvex bound with saving $\delta>0$. Then, as $|t| \rightarrow \infty$,

$$
\frac{1}{2 \pi i} \int_{\Re s=\frac{1}{2}} H(s) R_{1}\left(\left|E\left(z, \frac{1}{2}+i t, \frac{1}{2}\right)\right|^{2}, s\right) d s=o(1) .
$$

Proof. We find, by (4-13), Proposition 4.5 combined with $U(s, w)^{-1}=O(1)$ when $\Re(s)=\Re(w)=\frac{1}{2}$, Lemma 2.22, Stirling's formula, Lemma A.5 and, finally, $1 / \zeta(1+i t)=O(\log |t|)$, that

$$
\begin{aligned}
R_{1}\left(\left|E\left(z, \frac{1}{2}+i t, \frac{1}{2}\right)\right|^{2}\right. & \left., \frac{1}{2}+i u\right) \\
& =O\left(|t|^{-\frac{1}{2}+\varepsilon} \max _{\chi, \chi^{\prime}}\left|Z_{\psi_{\tau}}\left(\frac{1}{2}+i(u+2 t), \frac{1}{2}+i(u-2 t), \chi, \chi^{\prime}\right)\right|\right) .
\end{aligned}
$$

Subconvexity implies that the max is

$$
O\left(\left((1+|u+2 t|)(1+|u-2 t|)(1+2|u|)^{2}\right)^{\frac{1}{4}-\delta}\right) .
$$

Using the rapid decay of $H(s)$ we finally obtain that

$$
J_{3}(t, \infty)=O\left(|t|^{-\frac{1}{2}+\varepsilon}|t|^{2\left(\frac{1}{4}-\delta\right)}\right)=o(1) .
$$

Remark 4.9. In the above proof we see that, as in the cuspidal case, the trivial bound from Theorem 2.17 only gives $O\left(|t|^{\frac{1}{2}+\varepsilon}\right)$. However, for a compact set $A$ the Maaß-Selberg relations easily yield

$$
\int_{A}\left|E\left(z, \frac{1}{2}+i t, \frac{1}{2}\right)\right|^{2} d \mu(z)=O(\log t) .
$$

To summarize, we have proved:

Theorem 4.10. Assume that for any $\chi, \chi^{\prime} \bmod 8$ the function $Z\left(s, 1-s, \chi, \chi^{\prime}\right)$ satisfies a subconvex bound. Then, as $|t| \rightarrow \infty$,

$$
\int_{\Gamma \backslash \mathbb{H}} F_{h}(z)\left|E_{\infty}\left(z, \frac{1}{2}+i t, \frac{1}{2}\right)\right|^{2} d \mu(z)=\frac{4}{\operatorname{vol}(\Gamma \backslash \mathbb{W})} H(1) \log |t|+o(\log |t|) .
$$

The asymptotics (1-10) and hence Theorem 1.6 now follow from Theorems 4.3 and 4.10 by an approximation argument as in [Luo and Sarnak 1995, p. 217]. 


\section{Appendix: Mellin transforms of products of Whittaker functions}

In this appendix we prove various bounds on Mellin transforms of products of Whittaker functions that we have not been able to find in the literature in the generality needed.

Lemma A.1. Let $p \in\{ \pm 1\}$. For $s=\frac{1}{2}+i t, w=1-s$, and $s_{0}$ fixed, we have the bound

$$
\frac{1}{\Gamma(w+p / 4)} \int_{0}^{\infty} W_{0, s_{0}-\frac{1}{2}}(y) W_{p / 4, w-\frac{1}{2}}(y) y^{s-1} \frac{d y}{y}=O\left((1+|t|)^{-\frac{1}{2}}\right)
$$

as $|t| \rightarrow \infty$.

Remark A.2. The estimate in Lemma A.1 cannot be improved, as the proof below shows that the estimate can be turned into an asymptotic rate of decay of the same order.

Proof. Using [Gradshteyn and Ryzhik 2007, 7.611 7., p. 821] we obtain

$$
\begin{aligned}
& \int_{0}^{\infty} W_{0, s_{0}-\frac{1}{2}}(y) W_{p / 4, w-\frac{1}{2}}(y) y^{s-1} \frac{d y}{y} \\
& =\frac{\Gamma\left(s+w-s_{0}\right) \Gamma\left(s+w+s_{0}-1\right) \Gamma(1-2 w)}{\Gamma(1-p / 4-w) \Gamma(s+w)} \\
& \quad \times{ }_{3} F_{2}\left(s+w-s_{0}, s+w+s_{0}-1, w-\frac{p}{4} ; 2 w, s+w ; 1\right) \\
& \quad+\frac{\Gamma\left(s-w+s_{0}\right) \Gamma\left(s-w-s_{0}+1\right) \Gamma(2 w-1)}{\Gamma(w-p / 4) \Gamma(s-w+1)} \\
& \quad \times{ }_{3} F_{2}\left(s-w+s_{0}, s-w-s_{0}+1,1-\frac{p}{4}-w ; 2-2 w, s-w+1 ; 1\right),
\end{aligned}
$$

if $\left|\Re\left(s_{0}-\frac{1}{2}\right)\right|+\left|\Re\left(w-\frac{1}{2}\right)\right|<\Re s$. The generalized hypergeometric series that appear in (A-1) converge for $\Re s<1+p / 4$. We now set $s=\frac{1}{2}+i t$ and $w=\frac{1}{2}-i t$ and get

$$
\begin{aligned}
& \int_{0}^{\infty} W_{0, s_{0}-\frac{1}{2}}(y) W_{p / 4,-i t}(y) y^{s-1} \frac{d y}{y} \\
& =\frac{\Gamma\left(1-s_{0}\right) \Gamma\left(s_{0}\right) \Gamma(2 i t)}{\Gamma\left(\frac{1}{2}-\frac{p}{4}+i t\right) \Gamma(1)}{ }_{3} F_{2}\left(1-s_{0}, s_{0}, \frac{1}{2}-\frac{p}{4}-i t ; 1-2 i t, 1 ; 1\right) \\
& \quad+\frac{\Gamma\left(s_{0}+2 i t\right) \Gamma\left(1-s_{0}+2 i t\right) \Gamma(-2 i t)}{\Gamma\left(\frac{1}{2}-\frac{p}{4}-i t\right) \Gamma(1+2 i t)} \\
& \quad \times{ }_{3} F_{2}\left(s_{0}+2 i t, 1-s_{0}+2 i t, \frac{1}{2}-\frac{p}{4}+i t ; 1+2 i t, 1+2 i t ; 1\right) .
\end{aligned}
$$


Using [Bailey 1964, p. 18], we infer that (see also [Jakobson 1994, (2.9), p. 1491])

$$
\begin{aligned}
{ }_{3} F_{2}\left(s_{0}+2 i t\right. & \left., 1-s_{0}+2 i t, \frac{1}{2}-\frac{p}{4}+i t ; 1+2 i t, 1+2 i t ; 1\right) \\
= & \frac{\Gamma\left(\frac{1}{2}+\frac{p}{4}-i t\right) \Gamma(1+2 i t)}{\Gamma\left(\frac{1}{2}+\frac{p}{4}+i t\right) \Gamma(1)}{ }_{3} F_{2}\left(\frac{1}{2}-\frac{p}{4}+i t, 1-s_{0}, s_{0} ; 1+2 i t, 1 ; 1\right),
\end{aligned}
$$

and thus

$$
\begin{aligned}
& \int_{0}^{\infty} W_{0, s_{0}-\frac{1}{2}}(y) W_{p / 4,-i t}(y) y^{s-1} \frac{d y}{y} \\
& =\frac{\Gamma\left(1-s_{0}\right) \Gamma\left(s_{0}\right) \Gamma(2 i t)}{\Gamma\left(\frac{1}{2}-\frac{p}{4}+i t\right)}{ }_{3} F_{2}\left(1-s_{0}, s_{0}, \frac{1}{2}-\frac{p}{4}-i t ; 1-2 i t, 1 ; 1\right) \\
& +\frac{\Gamma\left(s_{0}+2 i t\right) \Gamma\left(1-s_{0}+2 i t\right) \Gamma(-2 i t) \Gamma\left(\frac{1}{2}+\frac{p}{4}-i t\right)}{\Gamma\left(\frac{1}{2}-\frac{p}{4}-i t\right) \Gamma\left(\frac{1}{2}+\frac{p}{4}+i t\right)} \\
& \quad \times{ }_{3} F_{2}\left(1-s_{0}, s_{0}, \frac{1}{2}-\frac{p}{4}+i t ; 1+2 i t, 1 ; 1\right) .
\end{aligned}
$$

We want to understand the asymptotic behavior of the hypergeometric series appearing in (A-2). Since $\Re(s)=\frac{1}{2}<1+\frac{p}{4}$, these converge absolutely. Moreover, the only difference between the two series is the sign of $i t$, so that it suffices to treat the first series. The treatment of the second hypergeometric series appearing in (A-2) is similar. Using the series representation for ${ }_{3} F_{2}$ we see that

$$
{ }_{3} F_{2}\left(s_{0}, 1-s_{0}, \frac{1}{2}-\frac{p}{4}-i t ; 1-2 i t, 1 ; 1\right)=\sum_{n=0}^{\infty} \frac{\left(s_{0}\right)_{n}\left(1-s_{0}\right)_{n}\left(\frac{1}{2}-\frac{p}{4}-i t\right)_{n}}{(1)_{n}(1-2 i t)_{n}} \frac{1}{n !} .
$$

In order to determine its asymptotic behavior as $|t| \rightarrow \infty$ we want to interchange the summation with the limit, i.e., we want to take the limit $|t| \rightarrow \infty$ in each term of the series separately. For this, let $\epsilon \in\left(0 ; \frac{1}{4}\right)$ be sufficiently small and rewrite the terms appearing in (A-3) as

$$
\left|\frac{\left(s_{0}\right)_{n}\left(1-s_{0}\right)_{n}\left(\frac{1}{2}-\frac{p}{4}-i t\right)_{n}}{(1)_{n}(1-2 i t)_{n}}\right|=\left|\frac{\left(s_{0}\right)_{n}\left(1-s_{0}\right)_{n}}{(1+\epsilon)_{n}}\right|\left|\frac{(1+\epsilon)_{n}\left(\frac{1}{2}-\frac{p}{4}-i t\right)_{n}}{(1)_{n}(1-2 i t)_{n}}\right| .
$$

For $0 \leq l \leq n$ we have

$$
\left|\frac{(l+1+\epsilon)\left(l+\frac{1}{2}-\frac{p}{4}-i t\right)}{(l+1)(l+1-2 i t)}\right|^{2}=\frac{\left(l^{2}+\left(\frac{3}{2}-\frac{p}{4}+\epsilon\right) l+(1+\epsilon)\left(\frac{1}{2}-\frac{p}{4}\right)\right)^{2}+t^{2}(l+1+\epsilon)^{2}}{(l+1)^{4}+4 t^{2}(l+1)^{2}} .
$$

Since $2(l+1)>l+1+\epsilon$ and

$$
0 \leq l^{2}+\left(\frac{3}{2}-\frac{p}{4}+\epsilon\right) l+(1+\epsilon)\left(\frac{1}{2}-\frac{p}{4}\right) \leq(l+1)^{2},
$$


this implies that

$$
\left|\frac{\left(s_{0}\right)_{n}\left(1-s_{0}\right)_{n}\left(\frac{1}{2}-\frac{p}{4}-i t\right)_{n}}{(1)_{n}(1-2 i t)_{n}}\right| \leq\left|\frac{\left(s_{0}\right)_{n}\left(1-s_{0}\right)_{n}}{(1+\epsilon)_{n}}\right|
$$

for all $n \geq 0$. Furthermore, the hypergeometric series

$$
{ }_{2} F_{1}\left(s_{0}, 1-s_{0} ; 1+\epsilon ; 1\right)=\sum_{n=0}^{\infty} \frac{\left(s_{0}\right)_{n}\left(1-s_{0}\right)_{n}}{(1+\epsilon)_{n}} \frac{1}{n !}
$$

converges absolutely and therefore, by the theorem of majorized convergence, we finally obtain

$$
\lim _{|t| \rightarrow \infty}{ }_{3} F_{2}\left(s_{0}, 1-s_{0}, \frac{1}{2}-\frac{p}{4}-i t ; 1-2 i t, 1 ; 1\right)={ }_{2} F_{1}\left(s_{0}, 1-s_{0} ; 1 ; \frac{1}{2}\right) .
$$

Thus only the Gamma factors appearing in (A-2) determine the asymptotic behavior, and using Stirling's formula we see that

$$
\int_{0}^{\infty} W_{0, s_{0}-\frac{1}{2}}(y) W_{p / 4,-i t}(y) y^{s-1} \frac{d y}{y}=O\left(|t|^{-\left(\frac{1}{2}-\frac{p}{4}\right)} e^{-\frac{\pi}{2}|t|}\right)
$$

as $|t| \rightarrow \infty$. This implies the desired bound.

Lemma A.3. Let $p \in\{ \pm 1\}$. We have

$$
{ }_{3} F_{2}\left(\frac{1}{2}+\frac{p}{4}-i t, \frac{1}{2}+i u, \frac{1}{2}-i u ; 1,1-2 i t ; 1\right) \ll e^{\pi|u|}|u|^{-2 \epsilon}
$$

as $|u| \rightarrow \infty$, where the implied constant does not depend on $t$. Furthermore, there exists a constant $C$ independent of $t$ such that

$$
{ }_{3} F_{2}\left(\frac{1}{2}+\frac{p}{4}-i t, \frac{1}{2}, \frac{1}{2} ; 1,1-2 i t ; 1\right) \leq C .
$$

Proof. Since $\Re\left(2-2 i t-\left(1+\frac{1}{2}+\frac{p}{4}-i t\right)\right)>0$, the hypergeometric series ${ }_{3} F_{2}\left(\frac{1}{2}+\frac{p}{4}-i t, \frac{1}{2}+i u, \frac{1}{2}-i u ; 1,1-2 i t ; 1\right)$ converges. By the definition of the hypergeometric series we have

$$
{ }_{3} F_{2}\left(\frac{1}{2}+\frac{p}{4}-i t, s, 1-s ; 1,1-2 i t ; 1\right)=1+\sum_{m=1}^{\infty} \frac{(s)_{m}(1-s)_{m}}{(1)_{m} m !} \frac{\left(\frac{1}{2}+\frac{p}{4}-i t\right)_{m}}{(1-2 i t)_{m}}
$$

with $s=\frac{1}{2}+i u$. We now determine the behavior of the series as $|u| \rightarrow \infty$. We use the same argumentation that was already useful in the proof of Lemma A.1. We write

$$
\frac{(s)_{m}(1-s)_{m}}{(1)_{m} m !} \frac{\left(\frac{1}{2}+\frac{p}{4}-i t\right)_{m}}{(1-2 i t)_{m}}=\frac{(s)_{m}(1-s)_{m}}{(1+\epsilon)_{m} m !} \frac{\left(\frac{1}{2}+\frac{p}{4}-i t\right)_{m}(1+\epsilon)_{m}}{(1)_{m}(1-2 i t)_{m}}
$$


with $\epsilon>0$ sufficiently small. As before the second factor on the right-hand side can be bounded in norm by 1 , and it is straightforward to see that the first factor is real and positive, so

$$
\left|{ }_{3} F_{2}\left(\frac{1}{2}+\frac{p}{4}-i t, s, 1-s ; 1,1-2 i t ; 1\right)\right| \leq{ }_{2} F_{1}(s, 1-s ; 1+\epsilon ; 1) .
$$

The last hypergeometric function equals (see [Bailey 1964, (1), p. 2])

$$
\frac{\Gamma(1+\epsilon) \Gamma(\epsilon)}{\Gamma\left(\frac{1}{2}+\epsilon+i u\right) \Gamma\left(\frac{1}{2}+\epsilon-i u\right)},
$$

and the first statement now follows from Stirling's formula. The second statement follows from plugging $u=0$ in the above argument.

Remark A.4. A similar bound is given in [Jakobson 1994], Claim 3.4, p. 1499.

Lemma A.5. Let $p \in\{ \pm 1\}$. For $u, t \in \mathbb{R}$ we have

$$
\frac{1}{\left|\Gamma\left(\frac{1}{2}+\frac{p}{4}+i t\right)\right|^{2}} \int_{0}^{\infty} y^{-\frac{1}{2}+i u}\left|W_{p / 4, i t}(y)\right|^{2} \frac{d y}{y}=O\left((1+|t|)^{-\frac{1}{2}}\right)
$$

as $|t| \rightarrow \infty$. The implied constant is uniform in $u$.

Proof. Set

$$
I_{p, t}(u):=\int_{0}^{\infty} y^{-\frac{1}{2}+i u}\left|W_{p / 4, i t}(y)\right|^{2} \frac{d y}{y} .
$$

Since $\left|I_{n, t}(u)\right| \leq I_{n, t}(0)$, we assume that $u=0$. By [Gradshteyn and Ryzhik 2007, Formula 7.611 7., p. 821] we get

$$
\begin{aligned}
& I_{n, t}(0) \\
& =\frac{\Gamma\left(\frac{1}{2}-2 i t\right) \Gamma\left(\frac{1}{2}\right) \Gamma(2 i t)}{\Gamma\left(\frac{1}{2}-\frac{p}{4}+i t\right) \Gamma\left(1-\frac{p}{4}-i t\right)} \times{ }_{3} F_{2}\left(\frac{1}{2}-2 i t, \frac{1}{2}, \frac{1}{2}-\frac{p}{4}-i t ; 1-2 i t, 1-\frac{p}{4}-i t ; 1\right) \\
& \quad+\frac{\Gamma\left(\frac{1}{2}+2 i t\right) \Gamma\left(\frac{1}{2}\right) \Gamma(-2 i t)}{\Gamma\left(\frac{1}{2}-\frac{p}{4}-i t\right) \Gamma\left(1-\frac{p}{4}+i t\right)} \times{ }_{3} F_{2}\left(\frac{1}{2}+2 i t, \frac{1}{2}, \frac{1}{2}-\frac{p}{4}+i t ; 1+2 i t, 1-\frac{p}{4}+i t ; 1\right) .
\end{aligned}
$$

It suffices to consider the first term since the second term differs from the first one only by the sign of $t$. Using the transformation formulae of [Bailey 1964, p. 18], as in the proof of Lemma A.1 we see that

$$
\begin{aligned}
{ }_{3} F_{2}\left(\frac{1}{2}-2 i t, \frac{1}{2}, \frac{1}{2}-\frac{p}{4}-i t ; 1-2 i t, 1-\frac{p}{4}-i t ; 1\right) \\
=\frac{\Gamma\left(1-\frac{p}{4}-i t\right) \Gamma\left(\frac{1}{2}\right)}{\Gamma\left(\frac{1}{2}-\frac{p}{4}-i t\right)}{ }_{3} F_{2}\left(\frac{1}{2}+\frac{p}{4}-i t, \frac{1}{2}, \frac{1}{2} ; 1,1-2 i t ; 1\right) .
\end{aligned}
$$


By the second part of Lemma A.3 the hypergeometric series is bounded and we find — by bounding all the Gamma functions using Stirling — that

$$
\left|I_{p, t}(0)\right|=O\left(\frac{\Gamma\left(1-\frac{p}{4}-i t\right)}{\Gamma\left(\frac{1}{2}-\frac{p}{4}-i t\right)} \frac{\Gamma\left(\frac{1}{2}-2 i t\right) \Gamma(2 i t)}{\Gamma\left(\frac{1}{2}-\frac{p}{4}+i t\right) \Gamma\left(1-\frac{p}{4}-i t\right)}\right)=O\left(e^{-\pi|t|}|t|^{-\frac{1}{2}+\frac{p}{2}}\right)
$$

as $|t| \rightarrow \infty$, which gives the result.

\section{Acknowledgements}

We thank Gautam Chinta, Adrian Diaconu and Valentin Blomer for useful discussions about multiple Dirichlet series. Also we thank the referees for several helpful comments.

\section{References}

[Bailey 1964] W. N. Bailey, Generalized hypergeometric series, Cambridge Tracts in Mathematics and Mathematical Physics 32, Stechert-Hafner, New York, 1964. MR 32 \#2625 Zbl 0011.02303

[Blomer 2011] V. Blomer, "Subconvexity for a double Dirichlet series", Compos. Math. 147:2 (2011), 355-374. MR 2012f:11170 Zbl 1228.11140

[Blomer et al. 2014] V. Blomer, L. Goldmakher, and B. Louvel, " $L$-functions with $n$-th-order twists", Int. Math. Res. Not. 2014:7 (2014), 1925-1955. MR 3190355

[Brubaker et al. 2004] B. Brubaker, A. Bucur, G. Chinta, S. Frechette, and J. Hoffstein, "Nonvanishing twists of GL(2) automorphic L-functions", Int. Math. Res. Not. 2004:78 (2004), 4211-4239. MR 2005h:11099 Zbl 1083.11030

[Bump et al. 1996] D. Bump, S. Friedberg, and J. Hoffstein, "On some applications of automorphic forms to number theory”, Bull. Amer. Math. Soc. (N.S.) 33:2 (1996), 157-175. MR 97a:11072 Zbl 0865.11043

[Chinta and Diaconu 2005] G. Chinta and A. Diaconu, "Determination of a GL 3 cuspform by twists of central L-values", Int. Math. Res. Not. 2005:48 (2005), 2941-2967. MR 2006k:11094 Zbl 1085.11026

[Davenport 2000] H. Davenport, Multiplicative number theory, 3rd ed., Graduate Texts in Mathematics 74, Springer, New York, 2000. MR 2001f:11001 Zbl 1002.11001

[Diaconu et al. 2003] A. Diaconu, D. Goldfeld, and J. Hoffstein, "Multiple Dirichlet series and moments of zeta and L-functions", Compositio Math. 139:3 (2003), 297-360. MR 2005a:11124 Zbl 1053.11071

[Dutta Gupta 1997] S. Dutta Gupta, "On the Rankin-Selberg method for functions not of rapid decay on congruence subgroups", J. Number Theory 62:1 (1997), 115-126. MR 98f:11041 Zbl 0872.11024

[Friedberg and Hoffstein 1995] S. Friedberg and J. Hoffstein, "Nonvanishing theorems for automorphic L-functions on GL(2)", Ann. of Math. (2) 142:2 (1995), 385-423. MR 96e:11072 Zbl 0847.11026

[Gradshteyn and Ryzhik 2007] I. S. Gradshteyn and I. M. Ryzhik, Table of integrals, series, and products, 7th ed., Elsevier/Academic Press, Amsterdam, 2007. MR 2008g:00005 Zbl 1208.65001

[Heath-Brown 1995] D. R. Heath-Brown, "A mean value estimate for real character sums", Acta Arith. 72:3 (1995), 235-275. MR 96h:11081 Zbl 0828.11040 
[Hoffstein and Kontorovich 2010] J. Hoffstein and A. Kontorovich, "The first non-vanishing quadratic twist of an automorphic $L$-series", preprint, 2010. arXiv 1008.0839

[Iwaniec 1997] H. Iwaniec, Topics in classical automorphic forms, Graduate Studies in Mathematics 17, Amer. Math. Soc., Providence, RI, 1997. MR 98e:11051 Zbl 0905.11023

[Iwaniec 2002] H. Iwaniec, Spectral methods of automorphic forms, 2nd ed., Graduate Studies in Mathematics 53, Amer. Math. Soc., Providence, RI, 2002. MR 2003k:11085 Zbl 1006.11024

[Iwaniec and Kowalski 2004] H. Iwaniec and E. Kowalski, Analytic number theory, American Mathematical Society Colloquium Publications 53, Amer. Math. Soc., Providence, RI, 2004. MR 2005h:11005 Zbl 1059.11001

[Jakobson 1994] D. Jakobson, "Quantum unique ergodicity for Eisenstein series on $\mathrm{PSL}_{2}(\mathbf{Z}) \backslash \mathrm{PSL}_{2}(\mathbf{R})$ ", Ann. Inst. Fourier (Grenoble) 44:5 (1994), 1477-1504. MR 96b:11068 Zbl 0820.11040

[Koblitz 1984] N. Koblitz, Introduction to elliptic curves and modular forms, Graduate Texts in Mathematics 97, Springer, New York, 1984. MR 86c:11040 Zbl 0553.10019

[Koyama 2000] S.-y. Koyama, "Quantum ergodicity of Eisenstein series for arithmetic 3-manifolds", Comm. Math. Phys. 215:2 (2000), 477-486. MR 2001m:11085 Zbl 0982.11030

[Lindenstrauss 2006] E. Lindenstrauss, "Invariant measures and arithmetic quantum unique ergodicity”, Ann. of Math. (2) 163:1 (2006), 165-219. MR 2007b:11072 Zbl 1104.22015

[Luo and Sarnak 1995] W. Z. Luo and P. Sarnak, "Quantum ergodicity of eigenfunctions on $\mathrm{PSL}_{2}(\mathbf{Z}) \backslash \mathbf{H}^{2}$ ", Inst. Hautes Études Sci. Publ. Math. 81 (1995), 207-237. MR 97f:11037 Zbl 0852.11024

[Mizuno 2005] Y. Mizuno, "The Rankin-Selberg convolution for Cohen's Eisenstein series of half integral weight”, Abh. Math. Sem. Univ. Hamburg 75 (2005), 1-20. MR 2006k:11081 Zbl 1082.11025

[Nelson et al. 2014] P. D. Nelson, A. Pitale, and A. Saha, "Bounds for Rankin-Selberg integrals and quantum unique ergodicity for powerful levels", J. Amer. Math. Soc. 27:1 (2014), 147-191. MR 3110797 Zbl 06228355

[Petridis et al. 2013] Y. N. Petridis, N. Raulf, and M. S. Risager, "Quantum limits of Eisenstein series and scattering states", Canad. Math. Bull. 56:4 (2013), 814-826. MR 3121690 Zbl 06231120

[Roelcke 1966] W. Roelcke, "Das Eigenwertproblem der automorphen Formen in der hyperbolischen Ebene, I", Math. Ann. 167:4 (1966), 292-337. MR 1513277 Zbl 0152.07705

[Sarnak 2011] P. Sarnak, "Recent progress on the quantum unique ergodicity conjecture", Bull. Amer. Math. Soc. (N.S.) 48:2 (2011), 211-228. MR 2012e:58062 Zbl 1234.58007

[Selberg 1989] A. Selberg, Collected papers, I, Springer, Berlin, 1989. MR 92h:01083 Zbl 0675.10001

[Shahidi 1988] F. Shahidi, "On the Ramanujan conjecture and finiteness of poles for certain $L$ functions”, Ann. of Math. (2) 127:3 (1988), 547-584. MR 89h:11021 Zbl 0654.10029

[Shimura 1973] G. Shimura, "On modular forms of half integral weight”, Ann. of Math. (2) 97 (1973), 440-481. MR 48 \#10989 Zbl 0266.10022

[Shimura 1975] G. Shimura, "On the holomorphy of certain Dirichlet series", Proc. London Math. Soc. (3) 31:1 (1975), 79-98. MR 52 \#3064 Zbl 0311.10029

[Shnirelman 1974] A. I. Shnirelman, "Ergodic properties of eigenfunctions", Uspehi Mat. Nauk 29:6(180) (1974), 181-182. In Russian. MR 53 \#6648 Zbl 0324.58020

[Soundararajan 2010a] K. Soundararajan, "Quantum unique ergodicity for $\mathrm{SL}_{2}(\mathbb{Z}) \backslash \mathbb{M}$ ", Ann. of Math.

(2) 172:2 (2010), 1529-1538. MR 2011j:11098 Zbl 1209.58019

[Soundararajan 2010b] K. Soundararajan, "Weak subconvexity for central values of $L$-functions", Ann. of Math. (2) 172:2 (2010), 1469-1498. MR 2011i:11077 Zbl 1234.11066 
[Soundararajan and Young 2010] K. Soundararajan and M. P. Young, "The second moment of quadratic twists of modular L-functions", J. Eur. Math. Soc. (JEMS) 12:5 (2010), 1097-1116. MR 2011g:11097 Zbl 1213.11165

[Sturm 1980] J. Sturm, "Special values of zeta functions, and Eisenstein series of half integral weight", Amer. J. Math. 102:2 (1980), 219-240. MR 82b:10033a Zbl 0433.10015

[Titchmarsh 1986] E. C. Titchmarsh, The theory of the Riemann zeta-function, 2nd ed., Clarendon Press, New York, 1986. MR 88c:11049 Zbl 0601.10026

[Truelsen 2011] J. L. Truelsen, "Quantum unique ergodicity of Eisenstein series on the Hilbert modular group over a totally real field”, Forum Math. 23:5 (2011), 891-931. MR 2836373 Zbl 1282.11047

[Colin de Verdière 1985] Y. Colin de Verdière, "Ergodicité et fonctions propres du laplacien", Comm. Math. Phys. 102:3 (1985), 497-502. MR 87d:58145 Zbl 0592.58050

[Zagier 1981] D. Zagier, “The Rankin-Selberg method for automorphic functions which are not of rapid decay”, J. Fac. Sci. Univ. Tokyo Sect. IA Math. 28:3 (1981), 415-437. MR 83k:10056 Zbl 0505.10011

[Zelditch 1987] S. Zelditch, "Uniform distribution of eigenfunctions on compact hyperbolic surfaces", Duke Math. J. 55:4 (1987), 919-941. MR 89d:58129 Zbl 0643.58029

[Zelditch 1991] S. Zelditch, "Mean Lindelöf hypothesis and equidistribution of cusp forms and Eisenstein series", J. Funct. Anal. 97:1 (1991), 1-49. MR 92h:11046 Zbl 0743.58034

[Zelditch 1992] S. Zelditch, Selberg trace formulae and equidistribution theorems for closed geodesics and Laplace eigenfunctions: Finite area surfaces, Mem. Amer. Math. Soc. 465, Amer. Math. Soc., Providence, RI, 1992. MR 93a:11047 Zbl 0753.11023

Communicated by Peter Sarnak

Received 2012-10-05 Revised 2014-08-03 Accepted 2014-09-08

i.petridis@ucl.ac.uk Department of Mathematics, University College London, Gower street, London, WC1E 6BT, United Kingdom

raulf@math.univ-lille1.fr Laboratoire Paul Painlevé, U.F.R. de Mathématiques, Université Lille 1, Sciences et Technologies, 59655 Villeneuve d'Asqc Cédex, France

risager@math.ku.dk

Department of Mathematical Sciences, University of Copenhagen, Universitetsparken 5, DK-2100 Copenhagen $\varnothing$, Denmark 


\section{Algebra \& Number Theory}

msp.org/ant

\section{EDITORS}

MANAGING EDITOR

Bjorn Poonen

Massachusetts Institute of Technology

Cambridge, USA

\author{
EDITORIAL BOARD CHAIR \\ David Eisenbud \\ University of California \\ Berkeley, USA
}

BOARD OF EDITORS

Georgia Benkart

Dave Benson

Richard E. Borcherds

John H. Coates

J-L. Colliot-Thélène

Brian D. Conrad

Hélène Esnault

Hubert Flenner

Edward Frenkel

Andrew Granville

Joseph Gubeladze

Roger Heath-Brown

Craig Huneke

Yujiro Kawamata

János Kollár

Yuri Manin

Barry Mazur

Philippe Michel

Susan Montgomery
University of Wisconsin, Madison, USA

University of Aberdeen, Scotland

University of California, Berkeley, USA

University of Cambridge, UK

CNRS, Université Paris-Sud, France

University of Michigan, USA

Freie Universität Berlin, Germany

Ruhr-Universität, Germany

University of California, Berkeley, USA

Université de Montréal, Canada

San Francisco State University, USA

Oxford University, UK

University of Virginia, USA

University of Tokyo, Japan

Princeton University, USA

Northwestern University, USA

Harvard University, USA

École Polytechnique Fédérale de Lausanne

University of Southern California, USA
Shigefumi Mori

Raman Parimala

Jonathan Pila

Anand Pillay

Victor Reiner

Peter Sarnak

Joseph H. Silverman

Michael Singer

Vasudevan Srinivas

J. Toby Stafford

Bernd Sturmfels

Richard Taylor

Ravi Vakil

Michel van den Bergh

Marie-France Vignéras

Kei-Ichi Watanabe

Efim Zelmanov

Shou-Wu Zhang
RIMS, Kyoto University, Japan

Emory University, USA

University of Oxford, UK

University of Notre Dame, USA

University of Minnesota, USA

Princeton University, USA

Brown University, USA

North Carolina State University, USA

Tata Inst. of Fund. Research, India

University of Michigan, USA

University of California, Berkeley, USA

Harvard University, USA

Stanford University, USA

Hasselt University, Belgium

Université Paris VII, France

Nihon University, Japan

University of California, San Diego, USA

Princeton University, USA

\section{PRODUCTION}

production@msp.org

Silvio Levy, Scientific Editor

See inside back cover or msp.org/ant for submission instructions.

The subscription price for 2014 is US $\$ 225 /$ year for the electronic version, and $\$ 400 /$ year ( $\$ 55$, if shipping outside the US) for print and electronic. Subscriptions, requests for back issues and changes of subscribers address should be sent to MSP.

Algebra \& Number Theory (ISSN 1944-7833 electronic, 1937-0652 printed) at Mathematical Sciences Publishers, 798 Evans Hall \#3840, c/o University of California, Berkeley, CA 94720-3840 is published continuously online. Periodical rate postage paid at Berkeley, CA 94704, and additional mailing offices.

ANT peer review and production are managed by EditFLOW ${ }^{\circledR}$ from MSP.

\section{PUBLISHED BY}

- mathematical sciences publishers

nonprofit scientific publishing

http://msp.org/

(C) 2014 Mathematical Sciences Publishers 


\section{Algebra \& Number Theory}

Volume $8 \quad$ No. $7 \quad 2014$

Double Dirichlet series and quantum unique ergodicity of weight one-half Eisenstein series

Yiannis N. Petridis, Nicole RaUlF and Morten S. Risager

Monodromy and local-global compatibility for $l=p$

ANA CARAIANI

Finite generation of the cohomology of some skew group algebras

VAN C. NGUYEN and SARAH WITHERSPOON

On the supersingular locus of the $G U(2,2)$ Shimura variety

BENJAMIN HOWARD and GEORgIOS PAPPAS

Poincaré-Birkhoff-Witt deformations of smash product algebras from Hopf actions on

1701

Koszul algebras

Chelsea Walton and Sarah Witherspoon

Highly biased prime number races

1733

DANIEL FIORILLI

Bounded gaps between primes with a given primitive root

1769

PAUL POLlACK 